\title{
1 Germline DNA replication shapes the recombination landscape in
}

\section{2 mammals}

3 Florencia Pratto ${ }^{1 *}$, Kevin Brick ${ }^{1 *}$, Gang Cheng ${ }^{1}$, Gabriel Lam ${ }^{1}$, Jeffrey M. Cloutier ${ }^{1}$, Daisy

4 Dahiya $^{1}$, Stephen R. Wellard ${ }^{2}$, Philip W. Jordan², R. Daniel Camerini-Otero ${ }^{1}$

$5{ }^{1}$ Genetics and Biochemistry Branch, NIDDK, NIH, Bethesda, Maryland, USA

6 2Department of Biochemistry and Molecular Biology, Johns Hopkins University Bloomberg

7 School of Public Health, Baltimore, MD 21205

8 *These authors contributed equally

\section{Summary:}

10 Genetic recombination generates novel trait combinations and understanding how 11 recombination is distributed across the genome is key to modern genetics. The PRDM9 protein

12 defines recombination hotspots, however megabase-scale recombination patterning is

13 independent of PRDM9. The single round of DNA replication, which precedes recombination in

14 meiosis, may establish these patterns, therefore we devised a novel approach to study meiotic

15 replication that includes robust and sensitive mapping of replication origins. We find that meiotic

16 DNA replication is distinct; reduced origin firing slows replication in meiosis and a distinctive

17 replication pattern in human males underlies the sub-telomeric increase in recombination. We

18 detected a robust correlation between replication and both contemporary and ancestral

19 recombination and found that replication origin density coupled with chromosome size

20 determines the recombination potential of individual chromosomes. Our findings and methods

21 have far-reaching implications for understanding the mechanisms underlying DNA replication,

22 genetic recombination, and the landscape of mammalian germline variation. 


\section{Keywords}

24 DNA replication, recombination, meiosis, germline, in-silico modelling, crossover, genome

25 stability, human reproduction

\section{Introduction}

27 Sexual reproduction uses a specialized cell division called meiosis, in which a single round of

28 DNA replication is followed by two cell divisions to create haploid gametes. Genetic

29 recombination in meiosis assures faithful segregation of chromosomes and establishes patterns

30 of genetic linkage and inheritance. Recombination is initiated by the formation of hundreds of

31 programmed DNA double-strand breaks (DSBs). In mice and humans, DSBs are targeted by

32 DNA sequence-specific binding of a meiosis-specific histone methyltransferase, PRDM9

33 (Baudat et al., 2010; Myers et al., 2010; Parvanov et al., 2010). Hundreds of PRDM9 alleles

34 exist (Berg et al., 2010; Buard et al., 2014) that primarily differ in the DNA-binding domain.

35 Thus, each variant may yield a distinct patterning of meiotic DSBs (Smagulova et al., 2016).

36 Nonetheless, megabase-scale similarities between individuals demonstrate that a broad-scale,

37 PRDM9-independent layer of control also shapes meiotic recombination (Davies et al., 2016;

38 Myers et al., 2005; Smagulova et al., 2011). The clearest manifestation of this is seen in the

39 elevation of meiotic DSBs in subtelomeric DNA of human males, independent of PRDM9

40 genotype (Pratto et al., 2014).

41 Since DNA replication determines genome structure (Klein et al., 2019) and immediately

42 precedes DSB formation in meiosis, we hypothesized that DNA replication may drive the

43 broad-scale regulation of recombination in mammals. Replication and recombination are

44 correlated in yeasts (Borde et al., 2000; Murakami and Nurse, 2001) and in barley (Higgins et

45 al., 2012). A mechanistic link has been demonstrated in baker's yeast, where a component of

46 the DSB machinery is activated by passage of the replication fork (Murakami and Keeney,

47 2014). This results in a spatio-temporal coordination between replication and DSB formation.

48 All current knowledge of DNA replication in mammalian meiosis stems from classical papers

49 using early molecular and cytological techniques (for review see (Chandley, 1986)). The paucity

50 of studies of DNA replication in mammalian meiosis stems from the requirement to study

51 meiosis in-vivo, in the context of a complex tissue (Handel et al., 2014). This contrasts with 
52 cell-types that can be studied in vitro; indeed, mammalian DNA replication is exclusively studied

53 in cell culture. This presents difficulties in adapting techniques for studying replication, which

54 have been mostly designed and optimized for cell culture. To address this shortcoming, we

55 devised the first method to map origins of replication in mammalian tissue (in this case, testis),

56 developed a cell-type specific method to interrogate replication timing in meiotic S-phase and

57 designed an in-silico modelling strategy to parameterize DNA replication in-vivo. This unique

58 tripartite approach has generated the first comprehensive, parameterized description of DNA

59 replication genome-wide in meiosis, or, for that matter, in any mammalian tissue. We found that

60 DNA replication in the germline is distinct from replication in other cell types and that this unique

61 replication patterning plays a direct role in shaping megabase-scale patterns of meiotic

62 recombination and genome diversity.

\section{Results}

\section{Highly specific replication origin mapping in mammalian testis}

65 To identify origins of replication, we introduced an important adaptation to an existing method to 66 sequence the RNA-primed short nascent leading strands (SNS) (Bielinsky and Gerbi, 1998)

67 (Figure 1A). Briefly, RNA-primed leading strands are isolated by using lambda exonuclease to 68 digest all DNA that lacks an RNA primer. Okazaki fragments, while also RNA-primed, are 69 excluded by size selection. A key improvement upon SNS-Seq (Cayrou et al., 2012; Fu et al., 70 2014; Jodkowska et al., 2019; Picard et al., 2014) is that we directly sequence the captured 71 single-stranded DNA (Khil et al., 2012) at origins. This retains the strand information of the 72 nascent strand DNA (Origin-derived Single-Stranded DNA Sequencing; Ori-SSDS) (Figure 1A) 73 and avoids biases resulting from random-priming and second-strand synthesis 74 (Sequeira-Mendes et al., 2019). Characteristic bidirectional replication at origins manifests as a 75 strand switch in sequenced reads. Distinct from SNS-Seq experiments, we can differentiate true 76 origins from non-specific signals (Figure 1A-C, S1, S2) by imposing a requirement for

77 leading-strand asymmetry at origins (see methods). Accounting for strand asymmetry 78 substantially reduces the number of replication origins estimated by SNS-Seq experiments 79 (40-50\%; Figure S3; (Cayrou et al., 2015)) because local peaks within SNS-Seq signals have 80 been spuriously defined as very closely-spaced replication origins (Figure S3). 
81 We detected 10,932, 12,100 and 13,082 origins in replicate experiments from testes of

82 individual male mice, demonstrating that our method is sufficiently sensitive to derive replication

83 origin maps from individual animals. Prior to our experiments, the number of cells required to

84 detect origins appeared to be prohibitive for in-vivo studies; for example, SNS-Seq typically

85 starts from $10^{9}$ exponentially growing cultured cells (Almeida et al., 2018). We used just $2 \times 10^{6}$

86 replicating cells (Kojima et al., 2019) for Ori-SSDS; this is 3-5 fold fewer than methods that

87 immunoprecipitate pre-replication complexes (i.e. ORC1/ORC2 ChIP-Seq (Miotto et al., 2016))

88 and orders of magnitude fewer than are required for the sequencing of Okazaki fragments

89 (Petryk et al., 2016). 94\% of origins in the smallest set were found in at least one of the other

90 two experiments (Figure S4) and origins unique to individual samples were weak (Figure 1D,

91 S5). No origins were detected in control experiments, either using non-replicating tissue (sperm)

92 or by hydrolyzing the leading strand RNA-primer (see above and methods) (Figure 1C, S1).

$9394 \%$ of replication origins detected by Ori-SSDS in testis are found in SNS-based origin maps

94 from other cell types (Mouse Embryonic Fibroblasts (MEFs) and Embryonic Stem (ES) cells;

95 Figure S6). Furthermore, data from Okazaki-fragment sequencing (in activated B-cells) shows a

96 switch in the polarity of Okazaki fragments around origin centers (Figure S6). These data

97 strongly suggest that Ori-SSDS accurately captures origins of replication from tissue and that

98 origins in testis mostly occur at sites that are also used as origins in other cell types.

99 Origin efficiency is a measure of the frequency with which an origin is used. We use the 100 sequencing depth of "correctly" oriented Ori-SSDS read-pairs to infer origin efficiency (see 101 methods). This efficiency varies $\sim 100$-fold and is highly correlated between replicates (Figure

102 1D, S3; Spearman $\left.R^{2}=0.65-0.74\right)$. Despite the fragment asymmetry around the origins, there is

103 considerable overlap of the Watson and Crick signals (Figure 1A, S1). Thus, while DNA

104 replication originates at discrete loci, the initiation window spans several hundred base-pairs at

105 the origin center. This contrasts with S. cerevisiae, where an $11 \mathrm{bp}$ motif defines a more precise

106 origin of replication (Broach et al., 1983; Stinchcomb et al., 1980). Together, these data show

107 that Ori-SSDS can identify the origins of replication genome-wide from mammalian tissue.

108 11,565 high-confidence origins, found in at least two of three replicates (Figure S4, S5), were

109 used for subsequent analyses.

110 Origins identified from mouse testis are unevenly distributed in the genome (Figure S7A) and 111 cluster in gene rich regions; $66 \%$ of origins occur within $1 \mathrm{~Kb}$ of a transcription start site (TSS) 
112 (Figure S7B). DNA at the origin center is intrinsically flexible, flanked by relatively rigid DNA, 113 and this may reflect a requirement of the ORC complex to bend DNA (Li et al., 2018) (Figure 114 S7C). Origins are GC-rich, with elevated CpG, and to a lesser extent, GpC dinucleotides (Figure 115 S7D). GC content, CpG density and GpC density are all positively correlated with origin 116 efficiency (Figure S7E) implying that nucleotide content either directly or indirectly plays a role in 117 origin firing. G-quadruplexes, a predicted secondary structure implicated in origin firing (Valton 118 et al., 2014), are also strongly enriched at origin centers (Figure S7F). 74\% of origins coincide 119 with CpG islands (CGIs) in accessible chromatin (as measured by ATAC-Seq in spermatogonia 120 - the germ-cells that precede meiosis (Maezawa et al., 2018); Figure 1E), yet only 53\% of such 121 sites are used for origin formation (Figure 1F). CGls in inaccessible chromatin and open 122 chromatin lacking a CGI are far less predictive of origin locations (Figure 1E,F). We did not 123 identify any conserved sequence motif at replication origins, however the density of CpG 124 dinucleotides alone can predict origin locations (Figure S7G). Indeed, CpG density is a better 125 predictor of origin locations than binding sites are for transcription factors (Figure S7H). Many of 126 these observations are consistent with properties of replication origins defined in cultured cells 127 (Marchal et al., 2019; Miotto et al., 2016), and together suggest that replication in the germline 128 initiates preferentially at CpG islands, near gene promoters, in accessible chromatin.

129 Broad replication initiation zones - up to $150 \mathrm{~Kb}$ and comprising $\sim 7 \%$ of the genome - have 130 been proposed as a major mode of replication initiation in humans (Petryk et al., 2016). 131 Although most origins mapped by Ori-SSDS are narrow and discrete, 1,925 Ori-SSDS peak are 132 wider than $10 \mathrm{~Kb}$, and may represent replication initiation zones (the expected width of the 133 Ori-SSDS signal is approximately $\pm 3 \mathrm{~Kb}$ around the origin of replication; determined by the size 134 selection step; see methods; Figure 1A; Figure S8A) To assess if putative initiation zones result 135 from closely-spaced, but difficult-to-resolve discrete origins, we examined the Ori-SSDS signal 136 around local peaks in CpG density (CpG peaks coincide with the center of isolated origins; 137 Figure S8B). We found that within our defined initiation zones, most local CpG peaks exhibited 138 origin-like Ori-SSDS strand asymmetry, implying that these sites are de-facto origins (Figure 139 S8C). 620 zones contained multiple CpG peaks $\left(N_{\mathrm{CpG} \text { Peaks }}=1,645\right)$, thus, closely-spaced and 140 difficult-to-resolve origins account for a large number of initiation zones (Figure S8D-F). Among 141 such zones is the extensively studied HoxA locus, where Ori-SSDS data suggests that $\sim 19$ 142 origins of replication occur within just $110 \mathrm{~Kb}$ (Figure S8E). 816 zones contained a single CpG 143 peak, and likely represent origins where we captured longer-than-expected nascent strands 
144 (Figure S8G). The remaining 490 zones did not contain any CpG peak above our threshold and 145 may represent more amorphous replication initiation regions. Alternatively, they may represent

146 clusters of weak origins (Figure S8G,H). Overall, we found extensive evidence that clustered, 147 yet discrete origins of replication can be mistaken for initiation "zones". Previous experiments 148 that identified "zones" lacked the resolution to detect this fine sub-structure.

\section{An unambiguous snapshot of replication in meiotic S-phase}

150 Stochastic origin firing and uneven origin density result in distinct earlier and later replicating 151 parts of the genome. We inferred this replication timing (RT) in meiosis. Meiotic S-phase nuclei 152 constitute just $1 \%$ of cells in adult testis (Kojima et al., 2019). Using a variant of fluorescence 153 activated nuclei sorting (Lam et al., 2019), we isolated up to $2 \times 10^{5}$ meiotic S-phase nuclei at 154 94\% purity (inspection of wide-field images; Figure S9) from a single adult mouse (Figure 2A-C); 155 this relied on a combination of DNA content, negative selection for a marker of non-meiotic cells 156 (DMRT1) and positive selection for a meiotic protein (STRA8). A marker not used for sorting, 157 but with expected meiotic expression (SYCP3) validated the purity of the meiotic population 158 (Figure 2C). We then inferred meiotic RT from coverage imbalances in whole-genome 159 sequencing of these nuclei (see methods; RT-Seq) (Koren et al., 2014). Replication origin 160 density is high in early replicating regions and low in late-replicating DNA (Figure 2D). This 161 yields the first snapshot of replication timing in mammalian meiosis. We also inferred RT from 162 the other major replicating cell types in adult testis - undifferentiated and differentiating 163 spermatogonia - and from published whole genome sequencing (WGS of other cell types (see 164 methods; Figure S10). Meiotic RT profiles are highly correlated among replicates and, to a 165 lesser extent, with RT from other cell types (Figure 2D-F, Figure S10). In particular, meiotic RT is 166 notably similar to RT in the cells that immediately precede meiotic S-phase and to RT in other 167 germ cells (PGCs, SSCs, ESCs). RT in all cell-types is highly correlated with GC-content 168 (Spearman $\mathrm{R}^{2}=0.27-0.40$ ) and with nuclear compartments (Spearman $\mathrm{R}^{2}=0.57$ 169 0.70)(Figure 2G).

\section{0 in-silico modeling recapitulates replication timing from origin locations}

171 RT-Seq yields a static snapshot of replication and these static RT-Seq snapshots from different 172 cell-types are often remarkably similar. Furthermore, the differences between RT-Seq profiles 173 are difficult to interpret because RT-Seq can be strongly influenced by the average stage of 
174 S-phase progression and by the relative synchrony of the population (Figure S11). We

175 hypothesized that subtle differences in the properties of replication among cell types could be

176 captured by in-silico modelling.

177 We built an in silico model of DNA replication that required experimentally defined origins as

178 input and that outputs a simulated RT profile. By examining the properties of replication that

179 produce a best-fit between simulated RT and experimental RT, we can understand the

180 parameters of replication that yield different RT profiles. Modeling parameters are the number of

181 active forks, whether to use origin efficiency estimates as a firing probability, and the total

182 duration of simulated S-phase (see methods). Replication fork speed is assumed constant

183 among cell types and throughout S-phase. After an initial round of simultaneous origin firing,

184 further origins fire only when two extant forks collide; this simulates the presence of factors that

185 limit the number of active origins (replisome ceiling), deemed important by previous simulations

186 of DNA replication (Gindin et al., 2014; Kelly and Callegari, 2019). Best fitting models were

187 obtained using a grid search for optimal model parameters (see methods).

188 We found that in silico modeling could accurately recapitulate experimental RT from both 189 meiotic (Figure 3A,B, Figure S12A) and non-meiotic S-phase cells (Figure 3B,C, Figure S12B).

190 In contrast, we could not obtain good-fitting models for non-replicating cells (2C) or when origin

191 locations were randomized (Ori(r); Figure 3B). Our success in modeling RT in all cell types

192 using testis-derived origins implies that a common set of origins are used during meiotic and

193 mitotic replication. Indeed in yeast, origins of replication are common to meiotic and mitotic cells

194 (Blitzblau et al., 2012; Wu and Nurse, 2014). Most testis-derived replication origins coincide with

195 CpG islands in open chromatin (ATAC-CGIs; Figure 1E,F), and these regions are broadly similar

196 across diverged cell types in mouse (Figure S13). RT can be modeled using ATAC-CGIs as a

197 proxy for origins, but these models are slightly worse than those using de facto origins (AS+CGI;

198 Figure 3B). Finally, RT-Sim does not explicitly distinguish between "early" and "late" firing 199 replication origins, suggesting that the paradigm of early and late origins is not required to 200 explain RT. Instead, "early replicating regions" and "late replicating regions" are defined by high 201 and low origin density, respectively. This is consistent with recent studies in other mammalian 202 cell types (Dileep and Gilbert, 2018; Gindin et al., 2014; Miotto et al., 2016; Takahashi et al., 203 2019). 


\section{Reduced origin firing slows DNA replication in meiosis}

205 To validate that RT-Sim can explain meaningful properties of replication, we examined model 206 run-time of best-fitting models for very early-, early-, middle- and late- S-phase nuclei (Figure 207 3D). Optimal model runtime - defined as the time at which simulated RT best fits experimental 208 RT - should reflect the time a population has spent in S-phase. Indeed, optimal runtime got 209 progressively longer from very early through late S-phase populations (Figure 3D), 210 demonstrating that modeling yields meaningful numeric insights into the properties of DNA 211 replication. Importantly, the differences between experimental RT in these populations do not 212 substantially affect model estimates of total S-phase duration (Figure 3D). Accurate estimates of 213 the duration of meiotic S-phase in mammals are notoriously difficult to obtain (Kofman-Alfaro 214 and Chandley, 1970); in mice, estimates range from 14 hrs (Monesi, 1962) to 29 hrs (Ghosal 215 and Mukherjee, 1971). Like in other organisms (Cha et al., 2000; Kofman-Alfaro and Chandley, 216 1970; Lee and Amon, 2001), DNA replication in meiotic S-phase is thought to be longer than 217 S-phase in non-meiotic cells (Kofman-Alfaro and Chandley, 1970). We find that for best-fitting 218 models, median S-phase duration in meiosis is $1.4-1.8$ fold longer than in spermatogonia - the 219 cells that precede meiosis (Figure 3E, S14), despite having highly correlated 220 experimentally-measured RT (Figure 2F). In Saccharomyces cerevisiae, the slow-down of DNA 221 replication in meiosis may be partly to facilitate recombination, as knocking out Spo11 - the 222 protein that makes meiotic DSBs - reduces S-phase duration by 30\% (Cha et al., 2000). Unlike 223 in yeast, we found no reduction in meiotic S-phase duration in Spo11\% mice (Figure S14).

224 The slow-down we observe in meiotic S-phase results from the use of fewer replisomes; 225 best-fitting models use just 0.24 - 0.28 replisomes $\mathrm{Mb}^{-1}$ in meiosis (648 - 756 replisomes per 226 haploid genome; 2,700 Mb genome), compared to $0.44-0.54$ replisomes $\mathrm{Mb}^{-1}$ in $\mathrm{ES}$ cells 227 (1,200 - 1,500 replisomes per haploid genome) (Figure 3F). These estimates in ES cells are 228 similar to the replisome count in cultured mouse C2C12 cells (imaging-based estimates; 0.46 2290.48 replisomes $\mathrm{Mb}^{-1}$; (Chagin et al., 2016)). The decreased replisome density in meiosis 230 mirrors findings in newt spermatocytes, where replication tracts in meiosis were notably longer 231 than in mitotic cells (Callan, 1973). Although we don't explicitly model fork speed, universally 232 altering fork speed without changing origin density cannot explain the presence of longer 233 replication tracts in meiosis. Indeed, in other organisms, fork speed does not vary between 234 meiosis and mitosis (Borde et al., 2000; Callan, 1973). By extrapolating from published 
235 estimates of S-phase duration in intermediate-stage spermatogonia (12.5 hr (Monesi, 1962)), 236 we estimate that meiotic S-phase in mice takes 21-24 hrs. Interestingly, the range of S-phase 237 duration estimates for the best-fitting models of meiotic $S$ phase is larger than that of the other 238 populations (Figure 3E, S14). Given that the model simulates replication in single cells, this 239 might indicate that there is a higher heterogeneity of RT profiles in meiosis vs mitosis. This is in 240 line with the experimental data spread seen in yeast (Cha et al., 2000).

241 Together, these analyses suggest both that the origins captured by Ori-SSDS reflect meiotic 242 replication initiation and that the same origins can yield tangibly different RT profiles if other 243 properties of replication vary. This tripartite approach of origin mapping, RT-Seq and in-silico 244 modeling, offers an alternative to classical cytogenetic approaches and yields an unprecedented 245 description of the DNA replication landscape.

\section{Direct coupling of replication with meiotic recombination}

247 Programmed DSB formation in meiosis occurs after DNA replication. In Saccharomyces 248 cerevisiae, passage of the replication fork favors meiotic DSB formation, likely through the 249 phosphorylation of Mer2 (a key protein for DSB formation) (Murakami and Keeney, 2014). This 250 interplay is completely unexplored in large mammalian genomes. In mice and humans, local 251 DSB patterning is determined by the sequence-specific binding of PRDM9 (Baudat et al., 2010; 252 Myers et al., 2010), yet at megabase scales, DSB density is highly correlated between 253 individuals with different PRDM9 alleles (Davies et al., 2016; Smagulova et al., 2011). We 254 therefore asked whether DNA replication underlies the megabase-scale control of DSB 255 patterning.

256 Meiotic DSBs form through a well-documented cascade; PRDM9 tri-methylates H3K4 (Brick et 257 al., 2012; Diagouraga et al., 2018) and/or H3K36 (Diagouraga et al., 2018; Powers et al., 2016) 258 which appear to recruit the DSB complex. A DSB is made by the SPO11 protein, which is then 259 released with a short oligonucleotide (Bergerat et al., 1997; Keeney et al., 1997; Neale et al., 260 2005). The 5' DNA is resected and the DMC1 protein loads on exposed ssDNA to facilitate 261 homologous recombination (Bishop et al., 1992). DSBs ultimately repair as a crossover (CO) or 262 as a non-crossover (NCO). H3K4me3 ChIP-Seq, SPO11-associated oligo mapping (Lange et 263 al., 2016), DMC1 ChIP-single-stranded DNA sequencing (Brick et al., 2012) and repair outcome 
264 mapping (COs/NCOs) provide differing quantitative readouts of intermediates in this cascade 265 (Figure 4A).

266 We found that all measures of recombination are enriched in the early replicating regions 267 (ERRs) (Figure 4). Nonetheless, informative differences are apparent. PRDM9-mediated 268 H3K4m3 and DSB frequency (SPO11-oligo) are similarly enriched relative to RT (Figure 4B; 269 yellow and magenta lines). Both profiles "flatten" in the earliest replicating DNA. This likely 270 reflects hotspot erosion (Boulton et al., 1997; Myers et al., 2010), a process by which strong 271 PRDM9 binding sites are purged from the genome (Figure 4C). Hotspot erosion is indicative of 272 ancestral recombination, reinforcing the strong association between RT and recombination in 273 mice. These findings also imply that the action of PRDM9 decouples replication and 274 recombination through the erosion of PRDM9 binding sites. In contrast to PRDM9-mediated 275 H3K4me3 and SP011-oligo density, DSB repair intermediates (DMC1-SSDS, RPA-SSDS) are 276 relatively depleted in the very earliest replicating regions (Figure 4B,D, S15). SSDS captures 277 both the frequency (Lange et al., 2016) and the lifetime (Pratto et al., 2014) of DSB repair 278 intermediates. Since DSB frequency closely mirrors PRDM9-mediated H3K4me3, we conclude 279 that DSBs forming in early replicating regions are more rapidly repaired than those elsewhere. 280 In agreement with this hypothesis, the signature of rapid repair is no longer seen in 281 DMC1-SSDS from a mouse in which all meiotic DSBs remain unrepaired (Hop2 ${ }^{-/}$; Figure S15).

282 The signature of rapid DSB repair in ERRs is also seen in mice that lack PRDM9 (Prdm9 $9^{-1-}$; 283 Figure S15). Thus, this phenomenon is independent of the mechanisms that determine the local 284 patterning of meiotic DSBs.

285 Rapid DSB repair in meiosis has been proposed as a hallmark of crossover-biased DSB repair 286 (Brick et al., 2018a; Hinch et al., 2019). To simplify the study of repair outcomes we turned to a 287 mouse homozygous for a "humanized" PRDM9 allele; this allele of PRDM9 has a binding 288 preference not found naturally in mice, and therefore, has left no footprint of hotspot erosion in 289 the mouse genome. Indeed, in these mice, PRDM9-mediated H3K4me3 is linearly correlated 290 with RT in the earliest replicating DNA (Figure 4D; yellow line), while the DMC1-SSDS signal 291 still shows a relative depletion indicative of rapid repair. We found that all inter-homolog repair 292 products (COs and NCOs) were notably depleted in the earliest replicating DNA, where rapid 293 DSB repair is occurring (Figure 4E, S15). The "missing" repair outcomes likely result from DSBs 294 that use the sister chromatid as a repair template, as inter-sister repair products cannot be 
295 detected (sister chromatids are genetically identical). Inter-sister DSB repair is generally

296 disfavored in meiosis to assure crossover formation between homologs. Nonetheless, this

297 inter-homolog bias appears to be gradually established, such that the earliest-forming DSBs can

298 still repair from the sister chromatid (Joshi et al., 2015; Sandhu et al., 2020). Given that we find

299 evidence for inter-sister repair of DSBs and given the fact that this type of repair is only favored

300 at the earliest-forming DSBs in meiosis, the implication of our findings is that DSBs at the

301 earliest replicating DNA are also formed early in meiosis. Other correlates of DNA replication,

302 such as GC-content or chromatin structure lack any mechanism that would link them to

303 early-forming DSBs. Therefore, the most parsimonious conclusion is that there exists a direct

304 link between replication and recombination in male mouse meiosis.

\section{Chromosome-scale regulation of recombination is coupled to DNA replication}

306 Chromosome-scale regulation of recombination is a PRDM9-independent aspect of 307 recombination patterning. For example, short chromosomes, by virtue of their size, are 308 statistically less likely to receive a crossover-competent DSB, yet mechanisms exist to assure 309 that they do (Duret and Galtier, 2009; Kaback et al., 1992; Murakami et al., 2020). In mouse 310 males, we find that short chromosomes have a higher crossover density (Figure 5A), however 311 much of the variance in crossover density remains unexplained. We hypothesized that DNA 312 replication may play a role because origin density varies greatly among mouse chromosomes; 313 for example, origin density on chromosome 13 (120 Mb; 327 origins) is three times lower than 314 on chromosome 11 (122 Mb; 1,023 origins). Indeed, we found that origin density is positively 315 correlated with crossover density (Figure S16). Origin density is a crude estimate of replication 316 activity that does not account for stochastic origin firing or competition between chromosomes

317 for limiting firing factors. We therefore derived a more integrated metric of replication from 318 RT-Sim models that measures how quickly chromosomes are replicated relative to each other 319 (replication speed; Figure 5B, S16). Alone, replication speed predicts crossover density 320 substantially better than origin density (Figure 5C, S16) and when coupled with chromosome 321 size in a multiple linear regression model can explain $90 \%$ of the per-chromosome variance in 322 crossover density (Figure 5D, S16). Strikingly, this implies that the recombination potential of 323 chromosomes is mostly established before DSB formation.

324 In yeast, compensation for chromosome size is achieved by increasing the DSB density on 325 short chromosomes (Murakami et al., 2020), but such an increase is not so apparent in mice 
326 (Lange et al., 2016). Whether differences in DNA replication modulate DSB formation or alter

327 DSB repair outcomes remains to be explored.

\section{Distinct sub-telomeric patterning of DNA replication in the human male germline}

329 In human males, like in mice, meiotic DSBs exhibit megabase-scale correlations across the 330 genome (Figure 6A). An overt manifestation of this phenotype is seen at the ends of human 331 chromosomes, where both DSBs (Pratto et al., 2014) and crossovers (Coop et al., 2008) are

332 grossly enriched in males, independent of PRDM9 genotype (Figure 6B).

333 We isolated S-phase nuclei expressing meiotic markers from human testes (Figure S17) and 334 inferred replication timing. Strikingly, we found that sub-telomeric regions of human 335 chromosomes replicate early and that therefore, germline RT is highly correlated with DSB 336 hotspot density $\left(R^{2}=0.75\right)$ (Figure $\left.6 B-C\right)$. This germline-specific RT patterning may explain why 337 only a weak link was previously found between RT in a lymphoblastoid cell line (LCL) and 338 meiotic recombination (Koren et al., 2012). The distal pattern of early replication in male meiosis 339 appears highly distinct, as sub-telomeric DNA does not replicate notably early in any of the 340 other cell types we studied (Figure 6C, S18). Intriguingly, GC-content is also elevated in 341 sub-telomeric DNA (Figure 6C,D); thus, genomic GC-content is better correlated with RT in the 342 germline than in other cell types (Figure 6D). Importantly, the earliest meiotic DSBs in human 343 males are detected almost exclusively in sub-telomeric DNA (Pratto et al., 2014). Thus, early 344 DNA replication in distal regions in the germline underlies the spatio-temporal patterning of 345 meiotic recombination in human males.

\section{Discussion}

347 The single round of DNA replication that begins meiosis has remained an enigma, despite huge

348 advances in our ability to interrogate nuclear processes. Aside from a cadre of classical papers 349 in the 1970s, few studies have addressed the intricacies of meiotic DNA replication in mammals 350 at a molecular level. We have addressed this shortcoming by developing a comprehensive 351 framework for the study of DNA replication in vivo and have used this to generate the first major 352 advances in the study of DNA replication in mouse and human meiosis in decades.

353 A major hurdle to overcome was to map origins of replication in the few S-phase cells that are 354 found in the mammalian testis. Existing protocols to map origins are designed for the controlled 
355 environment of cell culture, typically require tens to hundreds of millions of cells, and have not

356 been performed in tissue. By adapting methods for nascent-strand sequencing to retain the

357 directionality of captured strands, we generated a high-confidence map of $\sim 11,500$ origins of

358 replication in the testis. This is also the first map of in-vivo replication origins in a mammal and

359 paves the way for future studies in other cell types. Replication origins appear to be determined

360 by the combination of open chromatin and CpG density and are unevenly distributed in the

361 genome. CpG islands in open chromatin are broadly conserved across cell types, and indeed,

362 we find that the origins identified in testis represent a core set of cell-type-agnostic replication

363 origins. This does not negate the possibility of cell-type specific origins, as have been reported

364 in other studies (Smith et al., 2016).

365 Despite using the same population of replication origins, we exposed fundamental differences in 366 S-phase duration among cell-types through in-silico modelling. Importantly, experimental

367 methods to infer S-phase duration are laborious and imprecise or are limited to cell culture 368 systems (Grant et al., 2018). Meiotic S-phase has been suggested to be notably long in a 369 variety of organisms, including animals, plants, and yeast (Bennett et al., 1972; Callan, 1973; 370 Cha et al., 2000; Holm, 1977). Experimental estimates of S-phase duration in mice are 14-15 hr 371 and 29-30 hr in Spermatogonia-B and Spermatocytes, respectively (Ghosal and Mukherjee, 372 1971; Monesi, 1962). In agreement, we found that meiotic S-phase in mice is approximately 1.8 373 times longer than the germ-cells that immediately precede meiosis. The similarity between 374 in-silico and experimental estimates implies that the assumptions of our model are reasonable; 375 this includes the assumption that replication fork speed is similar across cell-types. To 376 accurately model replication, a limiting factor that caps the number of active replisomes is 377 strictly required ((Gindin et al., 2014) \& data not shown). We found that this replisome "ceiling" 378 differs among cell types, and, in turn, modulates S-phase duration. Importantly, such estimates 379 of the number of active replisomes per cell are extremely challenging to obtain experimentally 380 (Chagin et al., 2016). What components of the replication machinery govern this "ceiling" remain 381 unknown, but investigating the factors that slow down meiotic S-phase may help identify genes 382 that modulate S-phase duration more generally. In yeast, the meiosis-specific cohesin Rec8p 383 and DSB-forming protein Spo11p appear to play such a role. However, we found no evidence 384 for a role of SPO11 in regulating S-phase duration in mouse meiosis. In addition to a lowered 385 replisome "ceiling", other, more complex mechanisms may contribute to slowing S-phase in 386 meiosis. Our model only allows alteration of the global properties of replication, however 
387 regional modifiers of replication fork speed such as replication slow zones (Cha and Kleckner, 388 2002) or common fragile sites (Smith et al., 2006) may impede fork progression differentially in 389 meiosis and other cell types.

390 Our tripartite approach to describe and parameterize DNA replication enabled us to ask if 391 replication influences recombination in mammalian meiosis. We found an extremely strong 392 positive correlation between multiple metrics of recombination and early replicating DNA. The 393 earliest measure we examined was the binding of the PRDM9 protein, where we found that the 394 preference for DSB formation in early replicating DNA was already established. One possibility 395 therefore is that opportunistic binding of PRDM9 to more accessible chromatin in early 396 replicated DNA establishes a link between replication and recombination. However, we found 397 that recombination was enriched in early replicating DNA even in the absence of functional 398 PRDM9. This implies that broad-scale patterning of recombination acts independent of the 399 factors that determine the local patterning of meiotic DSBs.

400 We found strong evidence for the rapid repair of meiotic DSBs in early replicating parts of the 401 genome. The rapid repair of DSBs is a correlate of crossover-biased resolution (Hinch et al., 402 2019), however, we find that all interhomolog repair outcomes are depleted in these regions 403 suggesting increased use of the sister-chromatid as a repair template. In meiosis, DSB repair 404 with the sister chromatid is strongly disfavored. This assures that recombination occurs with the 405 homologous chromosome - a requirement for allelic exchange and accurate chromosome 406 segregation. At least in yeast, this inter-homolog bias is established gradually, and inter-sister 407 recombination is preferred only for the earliest DSBs that form in meiotic prophase (Joshi et al., 408 2015; Sandhu et al., 2020). We therefore infer that the earliest DSBs occur in the earliest 409 replicating DNA, strongly implicating a mechanistic link between replication timing per se and 410 DSB formation. This would also imply that correlates of replication timing such as GC-content, 411 genome compartmentalization, heterochromatin, or gene density, which lack any temporal 412 component, may indirectly affect recombination by modifying replication patterns. In human 413 males, cytological evidence has shown that the earliest meiotic DSBs occur almost exclusively 414 in sub-telomeric DNA (Pratto et al., 2014). This coincides with the earliest replicating DNA, 415 substantiating the direct link between replication and recombination initiation. It remains 416 possible that there are two phenomena that modulate recombination at large-scales in the 417 genome; one that temporally favors early-forming DSBs in the wake of a passing replication fork 
418 (like in yeast), and another that favors recombination in the resultant "permissive" chromatin 419 environment. Of course, since DNA replication establishes the 3D structure of the genome 420 (Klein et al., 2019), these two effects may be one-and-the-same.

421 It is well established that chromosome size partly determines per chromosome recombination 422 rates (Duret and Galtier, 2009; Kaback et al., 1992; Murakami et al., 2020). In addition, we 423 found that in mouse males, the per-chromosomes origin density has a similar predictive value to 424 chromosome size. Importantly, the predictive power of chromosome size and replication are 425 additive, which implies that these properties represent uncorrelated aspects of the mechanisms 426 that control per chromosome recombination rates. Together almost all of the per chromosome 427 variation in crossover density can be explained by these two properties, suggesting that 428 recombination will follow a deterministic path, established before DSBs are made. Short 429 chromosomes likely benefit from an elevated recombination rate to assure crossover formation, 430 however it is less intuitive to understand if or why elevated recombination on fast replicating 431 chromosomes would be beneficial. Fast-replicating chromosomes are origin rich, GC-rich, 432 replicate earlier, and have elevated gene density. A mechanism that links replication to 433 recombination would assure that such regions benefit from recombination to break linkage 434 blocks, generate diversity and purge deleterious mutations.

435 Any benefit of recombining in early replicating DNA could only manifest in the germline (where 436 meiotic recombination occurs) and intriguingly, in human males, germline DNA replication 437 mirrors GC content more closely than replication in other cell types. Thus, during development, 438 we propose that replication may follow a well-charted course, dictated by the underlying DNA. In 439 contrast, the commitment to differentiation in other cell types may render replication more 440 susceptible to epigenetic regulation (Hiratani et al., 2010). In being closely linked to GC content, 441 DNA replication may act as a "selectable trait" that influences the evolution of the genome. For 442 example, should a chromosome lack sufficient recombination capacity in meiosis, there would 443 be a strong selective advantage to increasing GC-content, leading to earlier replication and 444 hence, elevated DSB potential.

445 Errors in DNA replication, if unrepaired, can alter the genome, but will only be transmitted to the 446 next generation if they occur in the germline. Our finding that germline replication is substantially 
447 different therefore has important implications for understanding the patterning of de-novo

448 variation in the genome and its impact on population genetic structure.

\section{Acknowledgments}

450 We acknowledge all members of the Camerini-Otero lab for helpful discussion and comments.

451 We also thank Galina Petukhova, Michael Lichten and Peggy Hsieh for critiquing the 452 manuscript. We thank the Flow Cytometry Core of NHLBI, the NIDDK Laboratory of Animal 453 Sciences Section and the NIDDK genomics core facility. This work utilized the computational 454 resources of the NIH HPC Biowulf cluster (http://hpc.nih.gov). This work was funded by an 455 NIGMS grant to P.W.J. (R01GM11755) and by the intramural program of the NIDDK (R.D.C.O.). 456 We also thank the Washington Regional Transplant Community for their assistance in obtaining 457 de-identified human testis donations for research.

\section{Author Contributions}

459 Conceptualization, F.P., K.B. and R.D.C.O.; Methodology, F.P., K.B., G.L. and G.C.; 460 Investigation, F.P., K.B., G.L., D.D., J.C. and G.C.; Formal Analysis, F.P., K.B.; Software, K.B.; 461 Visualization, F.P., K.B.; Writing - Original Draft, F.P. and K.B.; Writing - Review \& Editing, F.P., 462 K.B. and R.D.C.O.; Resources, S.R.W. and P.W.J.; Funding Acquisition, P.W.J. and R.D.C.O.; 463 Supervision, R.D.C.O.

\section{Declaration of Interests}

465 The authors declare no competing interests.

\section{Data \& code availability}

467 The sequencing data have been deposited at the GEO (accession number GSE148327,

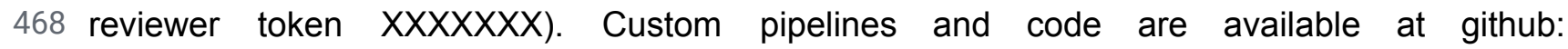
469 https://github.com/kevbrick/prattoEtAlAnalyticPipeline.git

\section{0 https://github.com/kevbrick/RTSeqPipeline.git}


bioRxiv preprint doi: https://doi ora/10.1101/2020 09 23,308874: this version posted September 23,2020 The copvriaht holder for this preprint (which was not certified by peer review) is the author/funder. This article is a US Government work. It is not subject to copyright under 17 USC 105 and is also made available for use under a CCO license.

471 https://github.com/kevbrick/RTsim.git 


\section{Figures}

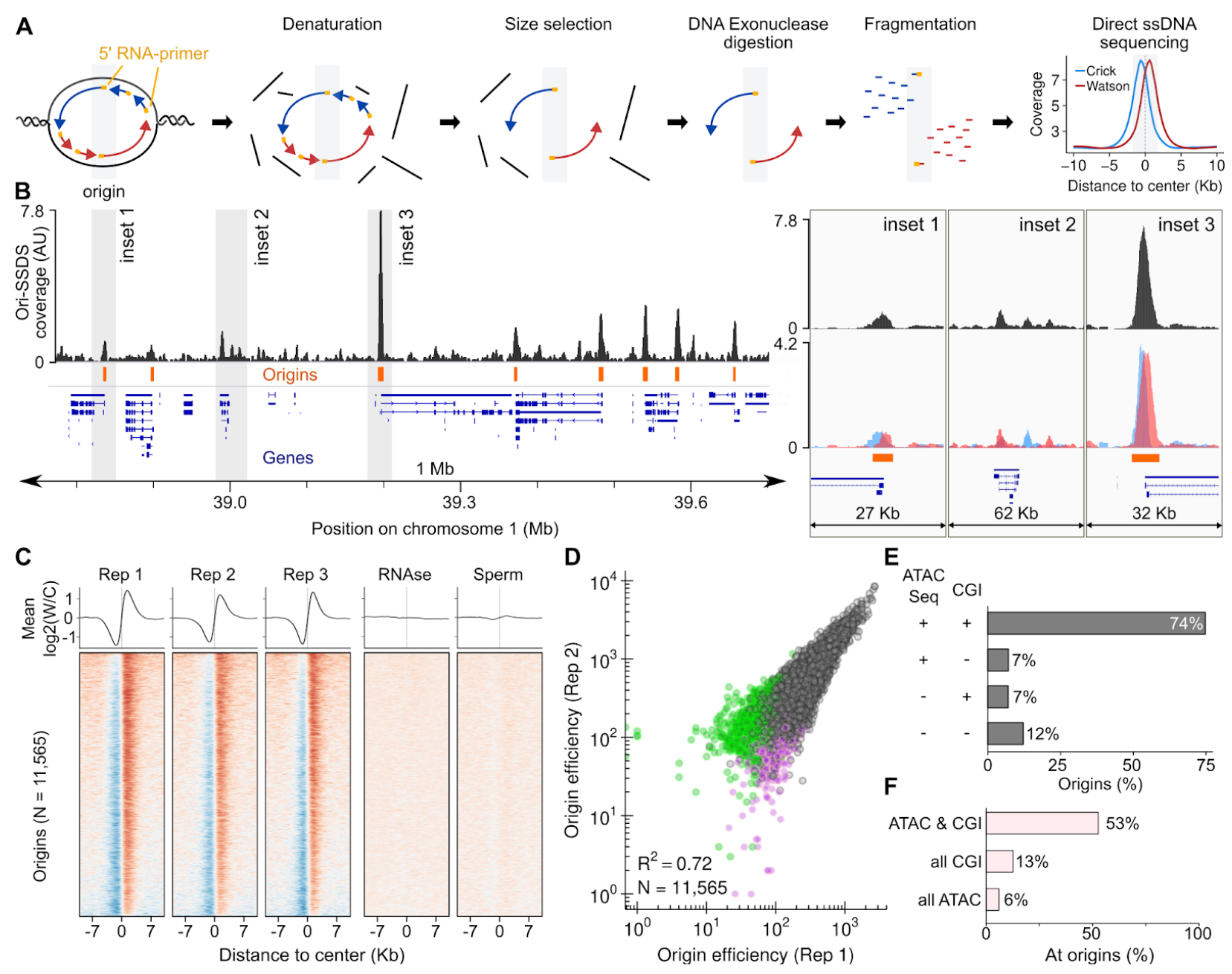

\section{Figure 1. Identification of replication origins}

474 (A) Schematic of the Ori-SSDS protocol to sequence nascent leading strands. (B) The 475 Ori-SSDS signal in a typical $1 \mathrm{Mb}$ region. Insets show the strand-specific Ori-SSDS signal at 476 representative weak (inset 1 ) and strong (inset 3 ) origins and at a series of non-origin peaks 477 (inset 2) that lack Ori-SSDS Watson-Crick asymmetry; red = Watson-strand coverage, blue = 478 Crick-strand coverage). Coverage is calculated in $1 \mathrm{~Kb}$ windows with a $147 \mathrm{bp}$ step. (C) 479 Ori-SSDS signal at origins is reproducible but is lost in control experiments. The $\log _{2}$ ratio of 480 Watson/Crick strand ssDNA fragments is shown, smoothed in $1 \mathrm{~Kb}$ windows. (D) Origin 481 efficiency is highly correlated among replicates. Origin efficiency varies $\sim 100$-fold. Origins found 
bioRxiv preprint doi: https://doi.org/10.1101/2020.09.23.308874; this version posted September 23, 2020. The copyright holder for this preprint (which was not certified by peer review) is the author/funder. This article is a US Government work. It is not subject to copyright under 17 USC 105 and is also made available for use under a CCO license.

482 only in one replicate are colored purple and green, respectively. (E) Origins of replication occur 483 predominantly at $\mathrm{CpG}$ islands (CGIs) that coincide with open chromatin as measured by 484 ATAC-Seq (Maezawa et al., 2018). (F) Origins are only detected at about half of the CGIs at 485 ATAC-Seq peaks. 

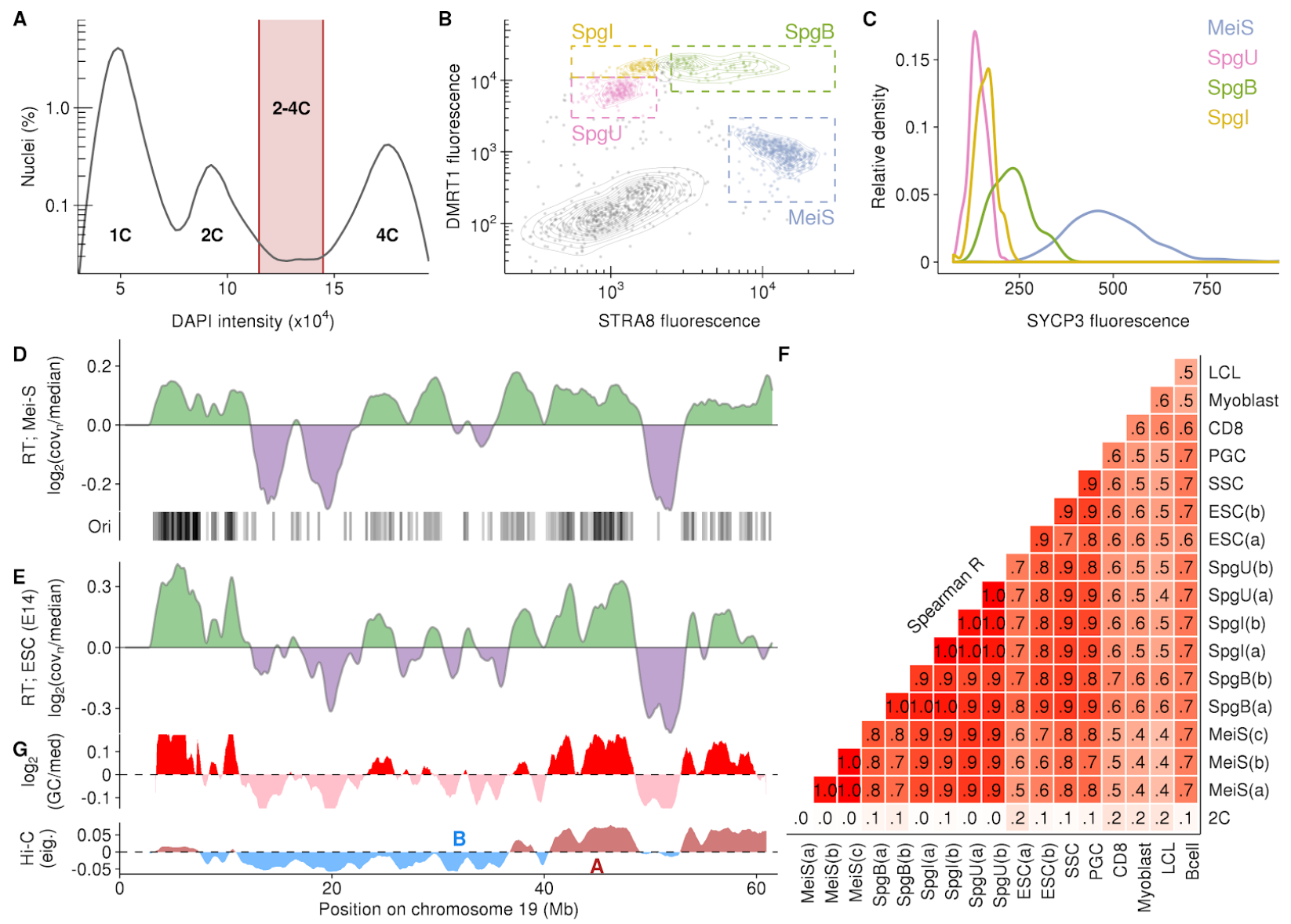

F
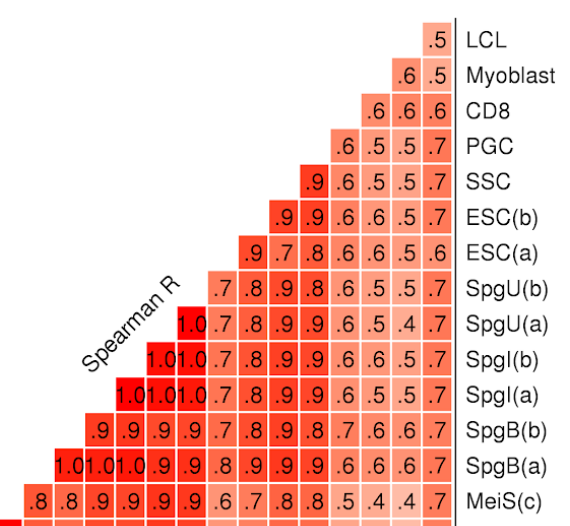

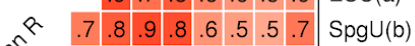

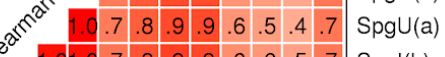
$5^{\mathrm{P}^{2}}$

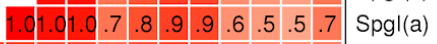

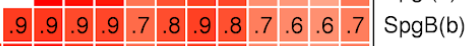
\begin{tabular}{|l|l|l|l|l|l|l|l|l|l|l|}
\hline 1.01 .01 .0 & .9 & .9 & .8 & .9 & .9 & .9 & .6 & .6 & .6 & .7 \\
\hline
\end{tabular}

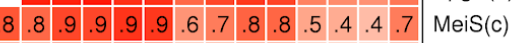
\begin{tabular}{|l|l|l|l|l|l|l|l|l|l|l|l|l|l|l|l|l|l}
\hline 1.0 & 8 & .7 & .9 & .9 & .9 & .9 & .6 & .6 & .8 & .7 & .5 & .4 & .4 & .7 & $\mathrm{MeiS}(\mathrm{b})$ \\
\hline
\end{tabular}

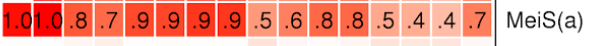

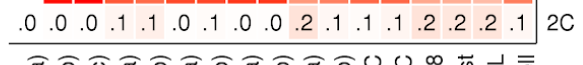

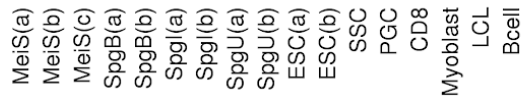

\section{Figure 2. Replication timing in meiotic prophase I of male mice}

487 (A-C) Meiotic S-phase and spermatogonia nuclei were isolated using fluorescence-activated 488 nuclei sorting (Lam et al., 2019). (A) Replicating nuclei were isolated by gating 2-4C nuclei by 489 DAPI content. (B) Meiotic S-phase nuclei (MeiS) can be distinguished from S-phase nuclei of 490 pre-meiotic germ cells using positive selection for nuclei expressing STRA8, and negative 491 selection for DMRT1. This sorting paradigm can also distinguish three sub-types of germ cells 492 (undifferentiated spermatogonia, SpgU; intermediate spermatogonia, Spgl; type-B 493 spermatogonia, SpgB) (C) SYCP3 is a meiosis-specific protein that was not used for nuclei 494 sorting. SYCP3 is elevated in the isolated meiotic nuclei relative to the other populations. 495 Above-background SYCP3 is also seen in the SpgB population. These are the germ-cells that 496 immediately precede meiotic entry. (D) Replication timing (RT; $\log _{2}$ of normalized sequencing 497 coverage $\left(\operatorname{cov}_{n}\right) /$ genome median) in meiotic S-phase nuclei correlates with origin locations in 
498 testis. (E) RT in E14 embryonic stem cells. (F) RT is highly correlated across cell-types. RT was

499 inferred from published whole genome sequencing data for Spermatogonial Stem Cells (SSC),

500 Primordial Germ Cells (PGC), Embryonic Stem Cells (ESC) CD8 ${ }^{+}$cells (CD8), and activated

501 B-cells (Bcell). Pre-processed RT data was obtained for Myoblast and Lymphoblastoid Cell

502 Lines (LCL). Details of samples in Tables S1-3. (G) GC-content (in 10Kb windows; shown as

$503 \log _{2}(\mathrm{GC} /$ meanGC); smoothed in $1 \mathrm{Mb}$ windows, $10 \mathrm{~Kb}$ steps) and genome compartmentalization

504 at zygonema (Patel et al., 2019) also correlate with RT. Hi-C track shows the eigenvector values

505 for the first principal component of the Hi-C matrix (calculated in $100 \mathrm{~kb}$ windows, smoothed in

$5061 \mathrm{Mb}$ windows, $10 \mathrm{~Kb}$ steps). Active $(\mathrm{A})$ and inactive $(\mathrm{B})$ compartments are highlighted. 

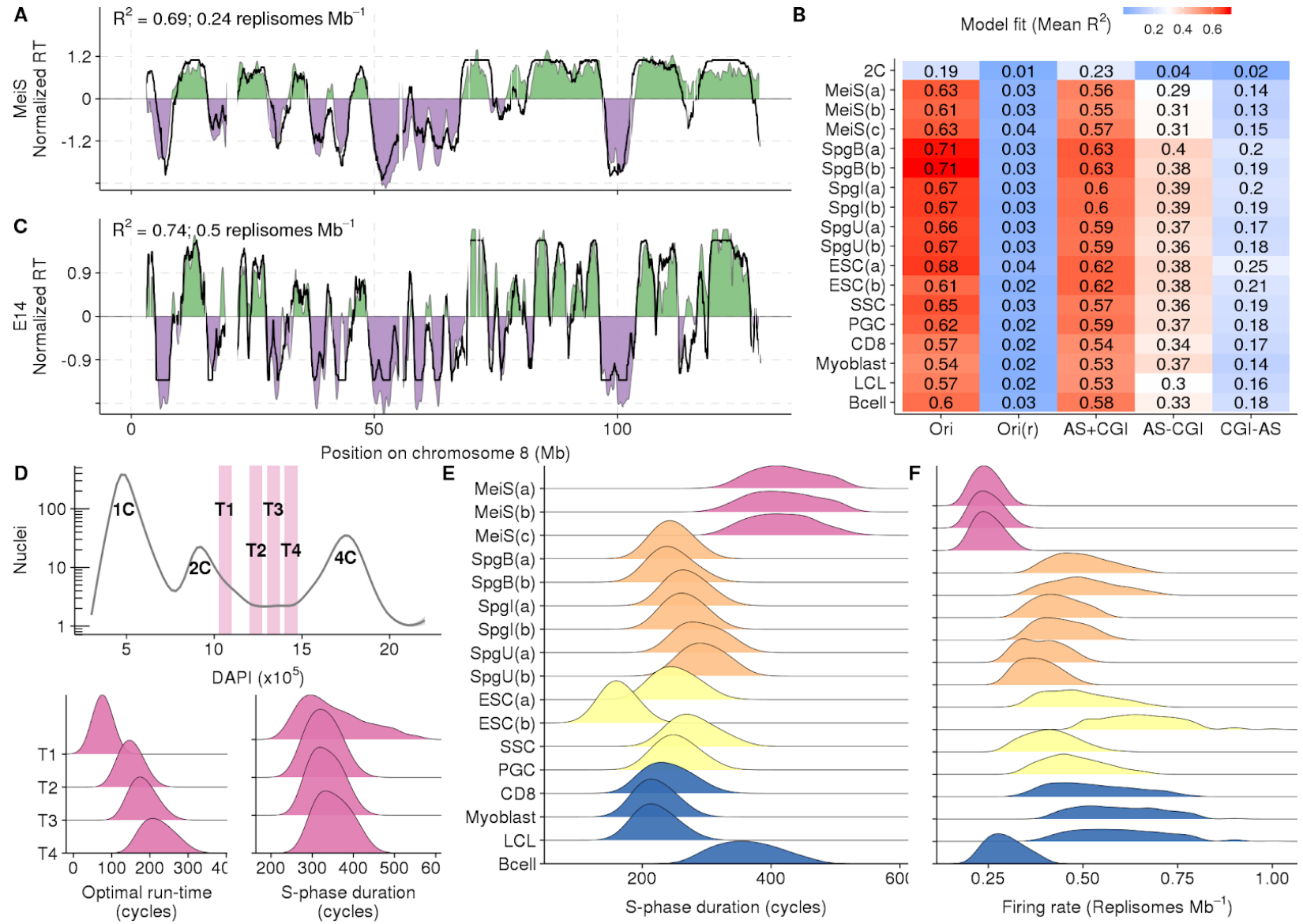

\section{Figure 3. In silico modeling recapitulates RT from origin locations}

508 (A) Best fitting RT-Sim model for Meiotic S-phase RT (MeiS(a)). Experimentally determined RT

509 is shown as filled area. Simulated RT is the black line. Both simulated and experimental RT are

510 normalized by mean and standard deviation (s.d.) (RTnorm = (RT-mean(RT)) / s.d.(RT)). (B) Fit

511 scores for models built using RT from different cell types and different datasets as a proxy for

512 origins. Mean of the top $0.015 \%$ of models is shown. Samples are described in detail in Tables

513 S1-3. Briefly, RT is from: MeiS = Meiotic S-phase, $\operatorname{Spg}(B, I, U)=B$-type, Intermediate and

514 Undifferentiated Spermatogonia, ESC = Embryonic Stem Cells, SSC = Spermatogonial Stem

515 Cells, $\mathrm{PGC}=$ Primordial Germ Cells, $\mathrm{CD} 8=\mathrm{CD} 8$ cells, Myoblast $=$ Myoblast cell line, $\mathrm{LCL}=$

516 Lymphoblastoid cell line, Bcell = Activated B-cells. $(a, b, c)$ designate replicates. ESC(a) is from

517 published whole genome sequencing data, $\operatorname{ESC}(b)$ is from cells grown by us. Ori = OriSSDS

518 origins, Ori( $r)=$ OriSSDS origins with randomized genomic location, $A S+C G I=$ ATAC-Seq peak

519 at a CGI, AS-CGI = ATAC-Seq peak not at a CGI, CGI-AS = CGI not at an ATAC-Seq peak. (C) 
520 Best fitting RT-Sim model for RT in E14 ESCs (ESC(a)). (D) Sorting schematic for S-phase

521 populations in lower panel. Populations have increasing DNA content from T1 to T4. The 522 optimal simulation run-time for best-fitting models correlates with increasing DNA content. 523 Nonetheless, the predicted S-phase duration is similar among all populations. (E) Total 524 replication time for best-fitting models. Meiotic S-phase is notably slow. (F) Best fitting models 525 imply that meiotic S-phase is slow because fewer replisomes fire than in other cell types. 526 Activated B-cells appear to share this property of replication with meiotic S-phase. 

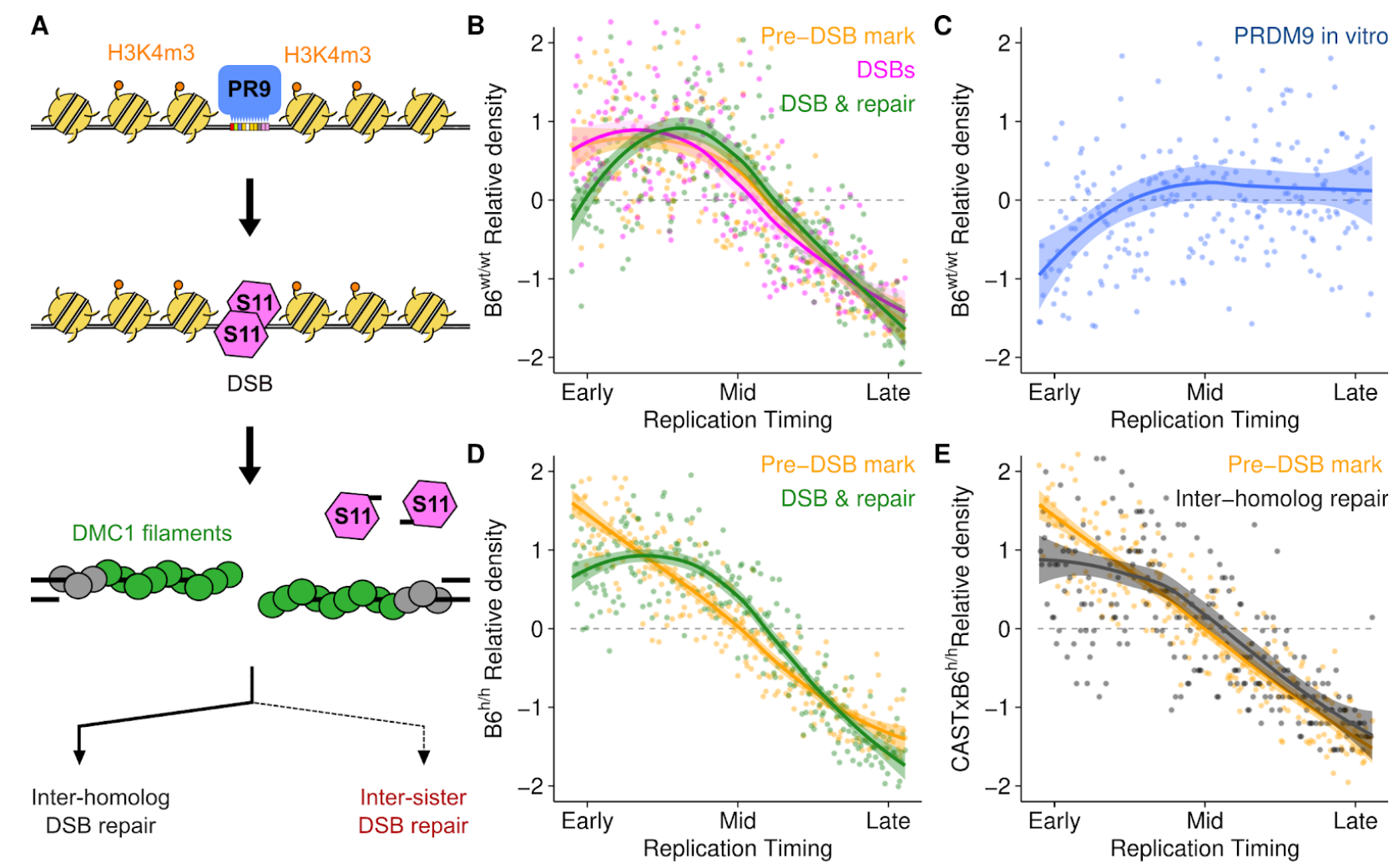

527 Figure 4. Rapid DSB repair and elevated meiotic recombination in early-replicating 528 regions

529 (A) Schematic of meiotic recombination. The colored lines in B-D reflect the colors in this 530 schematic. PR9 = PRDM9; S11 = SPO11. (B,D) PRDM9-mediated H3K4m3 at DSB hotspots 531 (yellow) is enriched in early replicating DNA in both wild-type B6 male mice (B6 ${ }^{\text {wtwt. }}$ B) and in 532 mice with a humanized PRDM9 allele (B6 $\left.{ }^{\mathrm{h} / \mathrm{h}} ; \mathrm{C}\right)$. DSB formation (SPO11-oligo mapping; pink) 533 follows this Pre-DSB mark (B). The DMC1-SSDS (green) signal decays relative to H3K4me3 in 534 the earliest replicating DNA. (C) B6 PRDM9 binding sites are depleted in early replicating DNA 535 (measured by Affinity-Seq) in the B6 $6^{\text {wtwt }}$ genome. This is indicative of hotspot erosion. (E) 536 Inter-homolog repair products (crossovers + non-crossovers; (Li et al., 2019a)) are depleted in 537 the earliest replicating DNA relative to DSB-associated $\mathrm{H} 3 \mathrm{~K} 4 \mathrm{~m} 3$ (Note that $\mathrm{H} 3 \mathrm{~K} 4 \mathrm{~m} 3$ are the 538 same data as in D, plotted against RT in B6xCAST). Simulated RT from the T1 Meiocyte 539 population (Figure 3D) is used for B,C and D. Simulated RT from the Meiotic S-phase in 540 B6xCAST F1 mice is used for E. Solid lines depict the LOESS smoothed signal \pm standard error 
bioRxiv preprint doi: https://doi.org/10.1101/2020.09.23.308874; this version posted September 23, 2020. The copyright holder for this preprint (which was not certified by peer review) is the author/funder. This article is a US Government work. It is not subject to copyright under 17 USC 105 and is also made available for use under a CCO license.

541 (shaded). For all panels, dots represent the average signal from all autosomal bins in the 542 genome for each RT quantile $\left(\mathrm{N}_{\text {bins }}=250\right)$. 

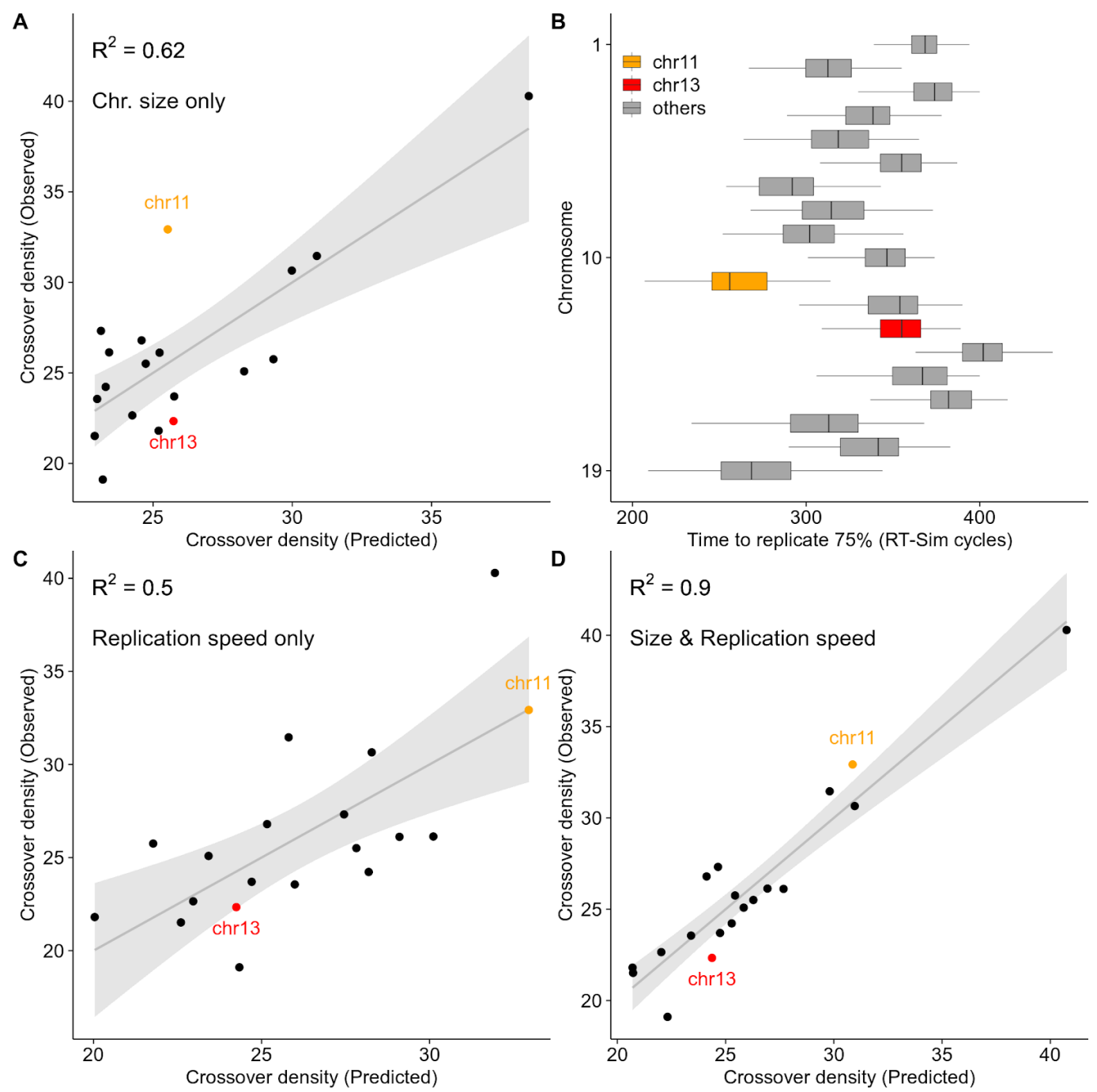

\section{Figure 5. DNA replication predicts per-chromosome crossover rates}

544 (A) A linear regression model can predict crossover density from chromosome size (polynomial 545 fit). Crossover density is calculated as total crossovers per Mb. Crossovers from (Yin et al., 546 2019). $R^{2}$ is strongly influenced by the single small outlier chromosome (chr19; rightmost). (B)

547 Chromosomes replicate asynchronously. Chromosome 11 and 13 are highlighted because while 548 they are a similar size, chromosome 11 replicates far earlier. The time required to replicate $75 \%$ 549 of each chromosome is inferred from the best-fitting RT-Sim model (see also Figure S16).

550 Boxplots indicate the range of times from individual modelled cells. (C) A linear regression 551 model can predict crossover density from replication speed. (D) Per chromosome crossover 
bioRxiv preprint doi: https://doi.org/10.1101/2020.09.23.308874; this version posted September 23, 2020. The copyright holder for this preprint (which was not certified by peer review) is the author/funder. This article is a US Government work. It is not subject to copyright under 17 USC 105 and is also made available for use under a CCO license.

552 density can be accurately predicted from a linear model that combines replication speed and 553 chromosome size. 

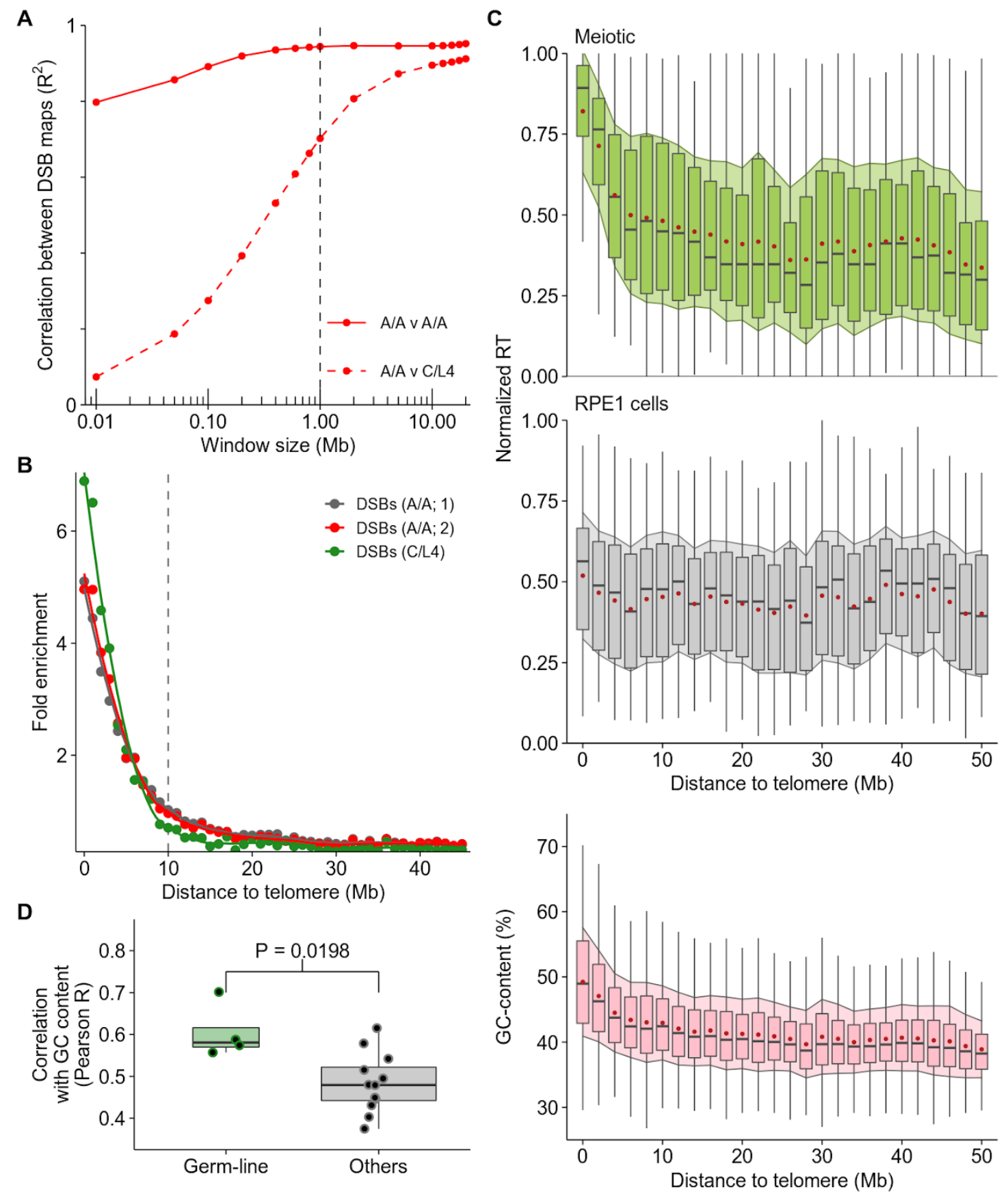

\section{Figure 6. Sub-telomeric DNA replicates early in human male meiosis}

555 (A) Extensive large-scale correlation in DSB density in human males with different PRDM9 556 genotypes (PRDM9A/A homozygotes (A/A) and PRDM9 ${ }^{C / L 4}$ (C/L4) heterozygote). At fine scale $557(10 \mathrm{~Kb})$, there is no correlation between DSB maps in the A/A and C/L4 individuals because 
558 DSB hotspot locations differ. Comparing the maps at lower resolution reveals extensive 559 correlation at megabase scales. (B) Meiotic DSBs are enriched in sub-telomeric regions in 560 human males, independent of the Prdm9 genotype. (C) Subtelomeric DNA replicates 561 consistently early in human male meiosis but not in mitotic (RPE-1; Table S3) cells. Boxplots 562 depict the range of replication timing values in $2 \mathrm{Mb}$ regions across the genome; the median is 563 shown as a grey bar and the box designates the interquartile range. The mean (red dot) \pm 1 564 standard deviation (filled shadow) is also shown for each interval. GC content is also elevated in 565 sub-telomeric DNA. (D) RT in the germline (all meiotic samples) correlates better with genomic 566 GC content than RT in other cell types. 


\section{Supplementary Figures}

A 10.0 - WT_Rep4
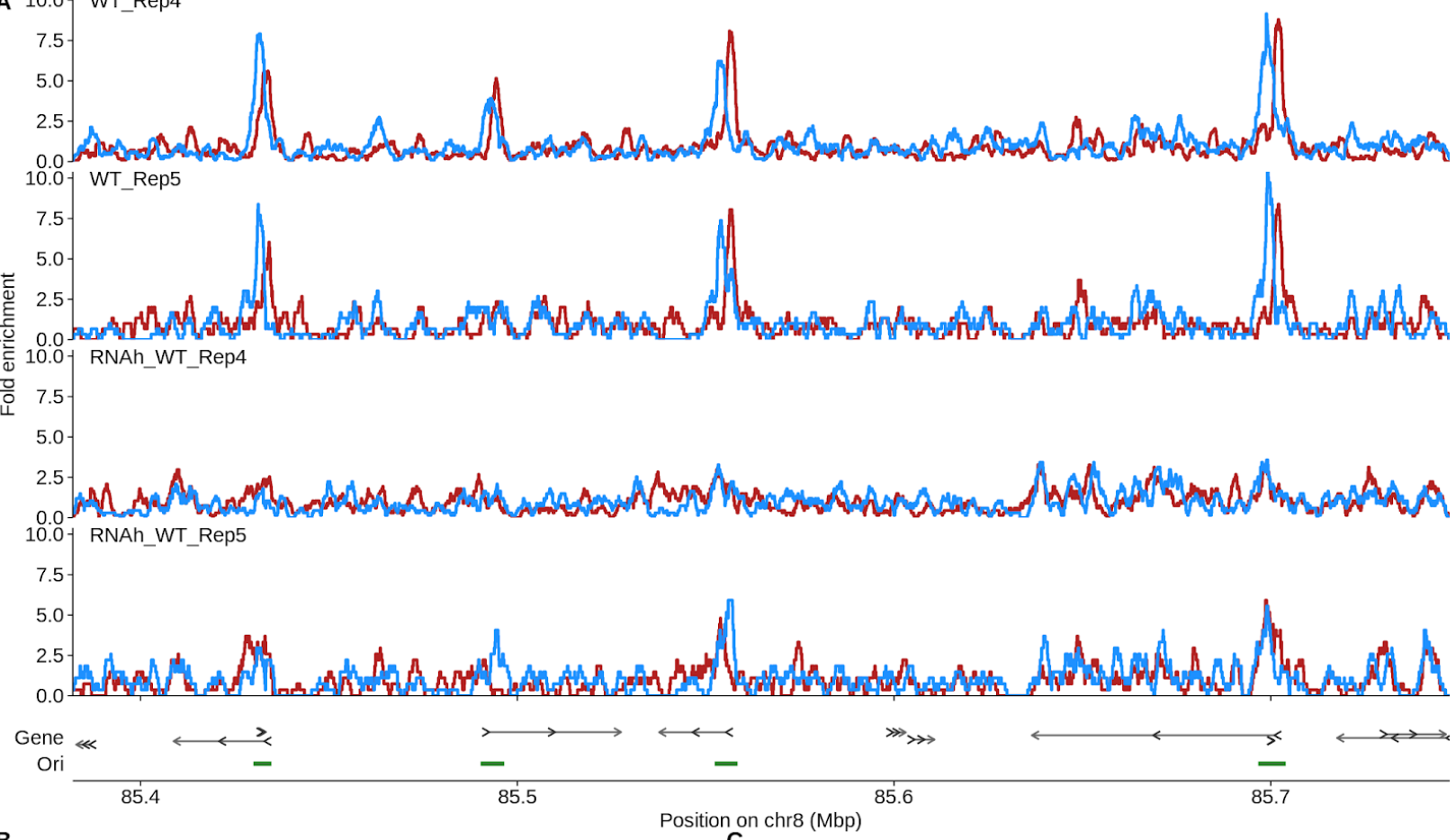

B
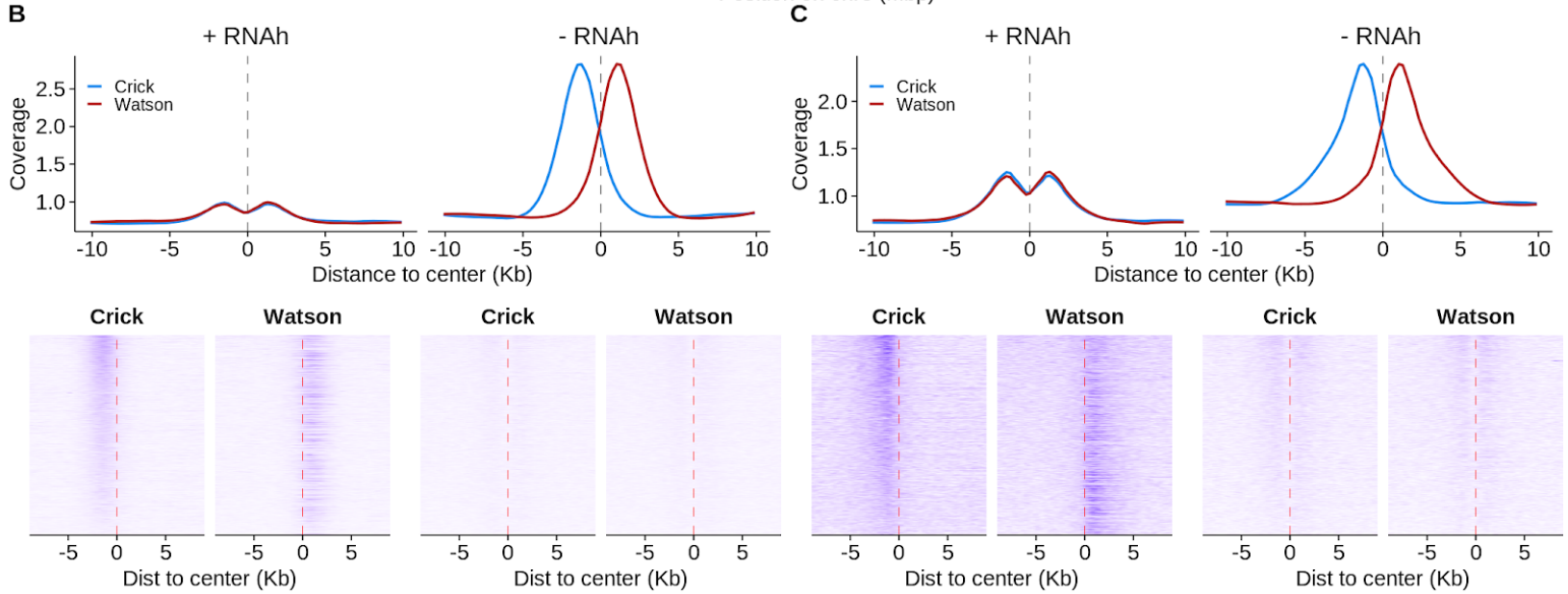

\section{Figure S1. RNA hydrolysis abolishes the Ori-SSDS signal.}

570 RNA hydrolysis prior to lambda exonuclease treatment abolishes Watson/Crick asymmetry at

571 origins of replication. This is because RNA hydrolysis will remove the nascent strand RNA

572 primer and thus render nascent-strands susceptible to lambda exonuclease digestion (see

573 methods). Two replicate experiments were performed in parallel; WT_Rep4 \& WT_Rep5 (see

574 methods) (A,B,C) The strand asymmetry of Ori-SSDS is lost following RNA hydrolysis (b,c 
bioRxiv preprint doi: https://doi.ora/10,1101/2020 09 23,308874: this version posted September 23,2020 The copvriaht holder for this preprint (which was not certified by peer review) is the author/funder. This article is a US Government work. It is not subject to copyright under 17 USC 105 and is also made available for use under a CCO license.

575 RNAh+ samples). Residual enrichment at origins, without Watson/Crick asymmetry is still seen.

576 This is likely because there is more DNA in the population at these sites as a result of DNA

577 replication. Upper panels in B,C depict the average Ori-SSDS signal at origins of replication for

578 Watson (red) and Crick (blue) strand reads. 


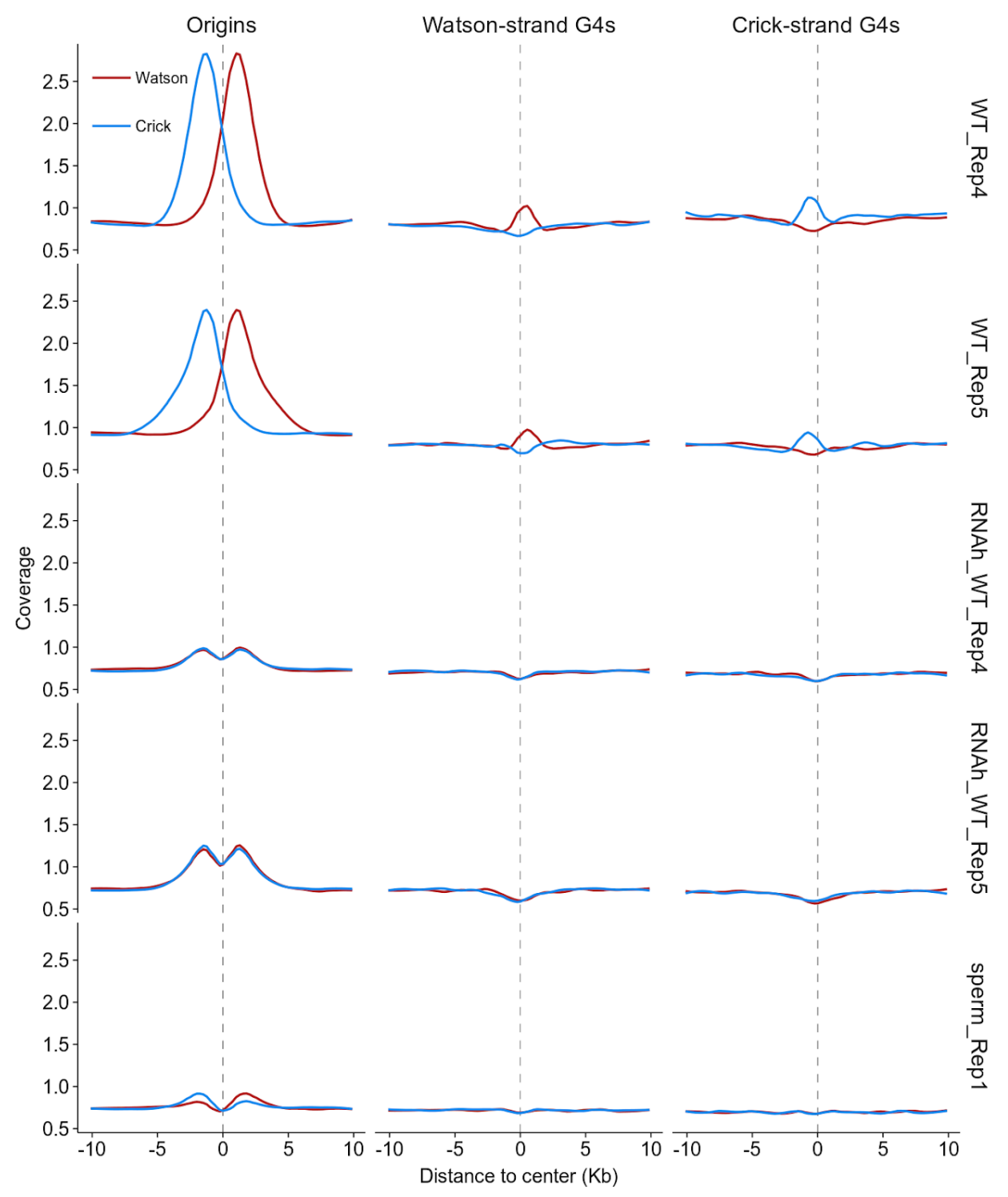

\section{Figure S2. Ori-SSDS signal at putative g-quadruplexes in replicating cells.}

580 G-quadruplexes (G4s) are secondary structures that have been hypothesized to impede lambda 581 exonuclease in vitro and may result in SNS-Seq peak artifacts (Foulk et al., 2015). Indeed, we 582 detect small peaks at putative G4 sequences in Ori-SSDS data. Figures depict the average 583 Ori-SSDS signal at origins of replication or predicted G-quadruplexes for Watson (red) and Crick 584 (blue) strand reads. Ori-SSDS sequencing can distinguish G4-signals from origin-derived 585 signals because G4s yield ssDNA on just one strand whereas reciprocal Watson/Crick 586 asymmetry is seen around the center of origins. This differentiates Ori-SSDS from other 587 SNS-Seq methods, which cannot distinguish G4s from origins. At origins that coincide with 
588 putative G4s (Huppert and Balasubramanian, 2005) it is not possible to resolve these signals

589 however, since the G4 signal is far weaker than that at origins, the contribution of G4s to true 590 origin signal is a minor concern. Curiously, the signal at G4s is not seen in non-replicating tissue 591 (sperm) or in samples where the RNA-primer is degraded. Thus, the G4-associated signal is 592 replication-dependent and not simply the result of G4 resistance to lambda exonuclease. The 593 stranded-ness of the signal is consistent with the capture of ligated Okazaki-fragments at the G4 594 sites. This implies that G-quadruplexes are an impediment to fork passage in-vivo. 
A
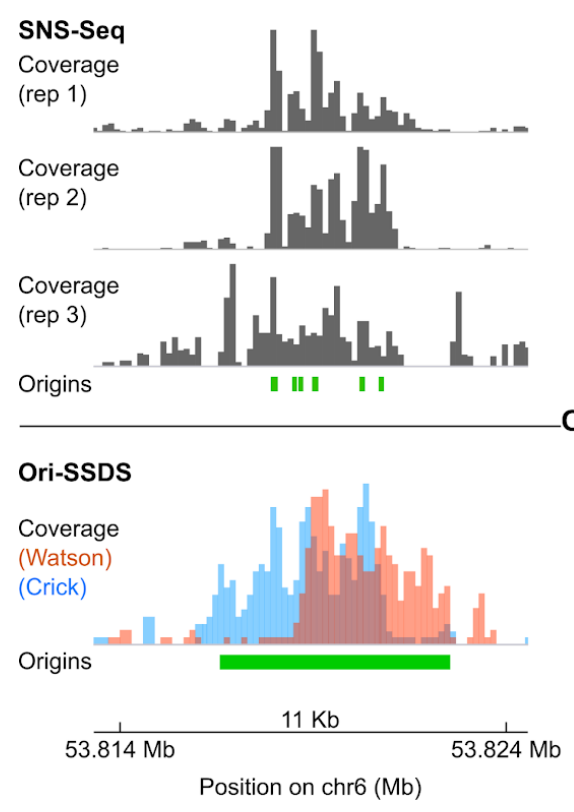

B

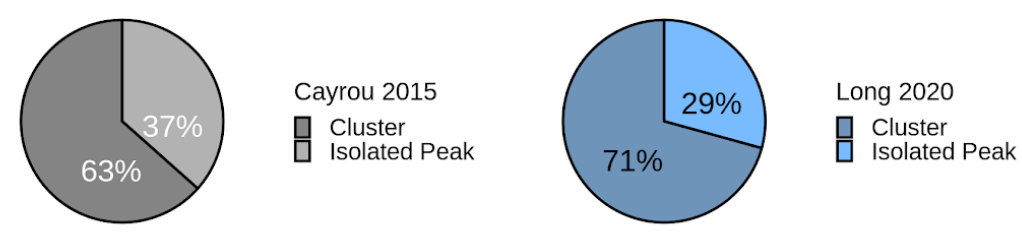

C

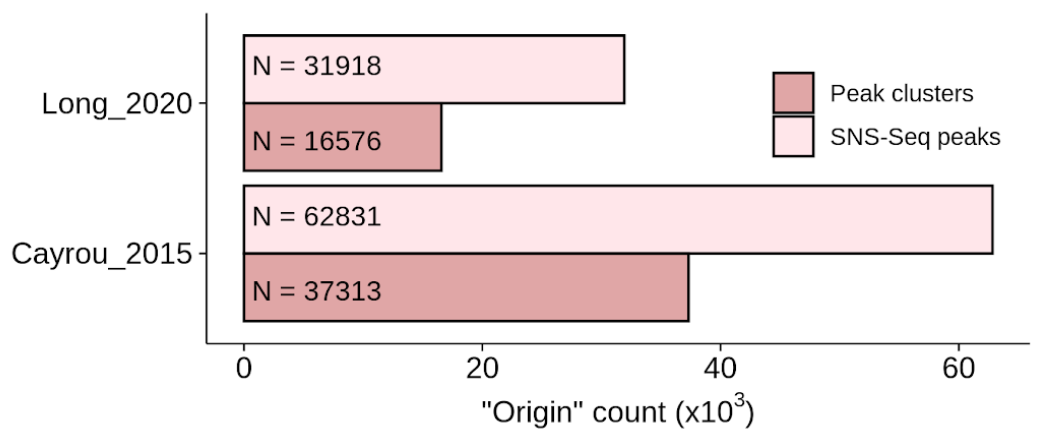

595 Figure S3. SNS-Seq peaks occur in clusters at individual origins of replication.

596 (A) Without knowledge of strand-asymmetry, SNS-Seq experiments have historically 597 overestimated the number of origins in the genome. At this locus, Ori-SSDS reveals that 6 598 SNS-Seq origin calls likely represent a single origin of replication. Grey tracks represent three 599 replicate SNS-Seq coverage tracks (Cayrou et al., 2015). Green boxes underneath the grey 600 tracks represent SNS-Seq origin calls (from GEO: GSE68347_Initiation_Sites.bedGraph.gz; 601 accession GSE68347; UCSC liftover was used to convert mouse mm9 to $\mathrm{mm} 10$ genome 602 coordinates) (Cayrou et al., 2015). Ori-SSDS coverage from WT Rep 1 is shown. The green 603 box under the Ori-SSDS track represents the single Ori-SSDS origin call in this region. This is 604 validated by the characteristic Watson-Crick asymmetry. (B) We obtained SNS-Seq origin of 605 replication peak calls (origins) for mouse (described in panel a.; (Cayrou et al., 2015)) and for 606 human (from GEO; GSE134988_siNC-NS_peaks.bed.gz; accession GSE134988) (Long et al., 607 2020). For each dataset, origins were extended $\pm 1.5 \mathrm{~Kb}$ from the center-point and merged into 608 origin "clusters"; clusters $>10 \mathrm{~Kb}$ were discarded. We posit that each of the remaining clusters 609 represents a single true origin of replication. Most SNS-Seq peaks occurred in a cluster. Just $61037 \%$ and $29 \%$ occurred at isolated origins in mouse and human, respectively. (C) The number 
bioRxiv preprint doi: https://doi.org/10.1101/2020.09.23.308874; this version posted September 23, 2020. The copyright holder for this preprint (which was not certified by peer review) is the author/funder. This article is a US Government work. It is not subject to copyright under 17 USC 105 and is also made available for use under a CCO license.

611 of "origins of replication" from SNS-Seq studies is substantially reduced if clusters are 612 considered as single origins. 


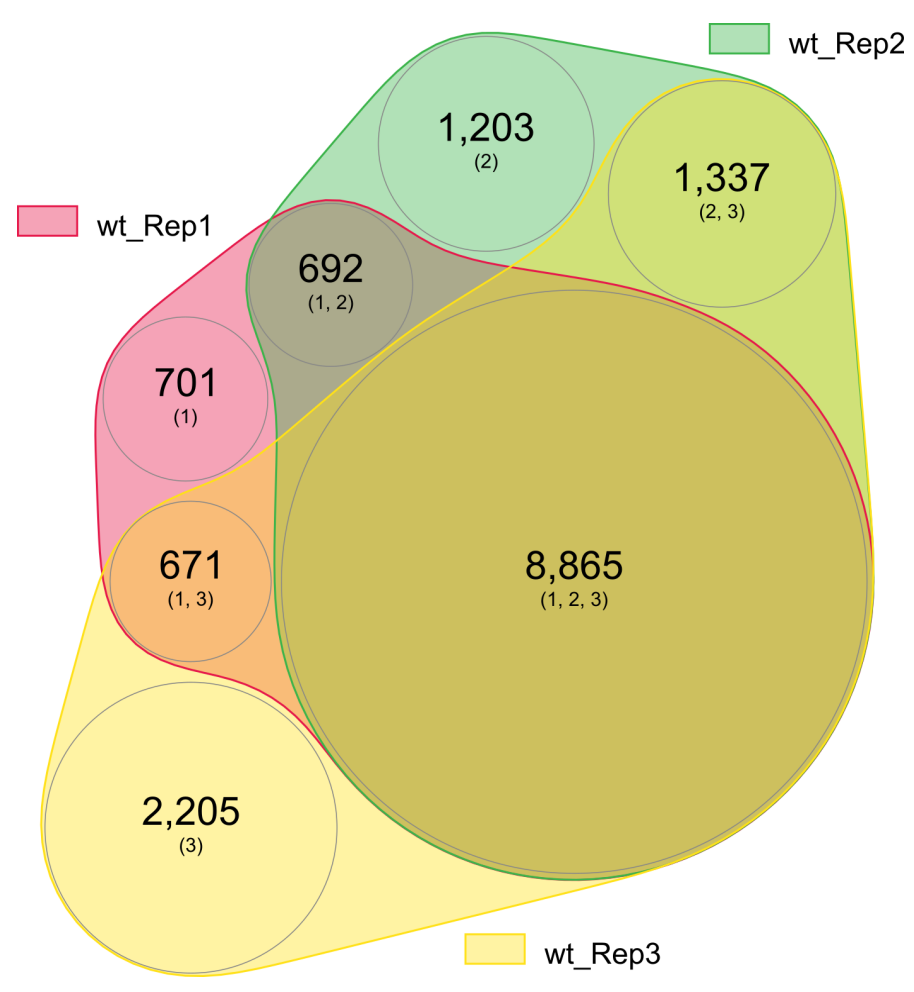

613 Figure S4. Most origins are detected in multiple replicate experiments

614 Proportionate venn diagram depicting the number of origins detected in each of three replicate

615 Ori-SSDS experiments and the overlaps between sets. Our consensus set of origins includes all 616 origins found in at least two Ori-SSDS experiments. 

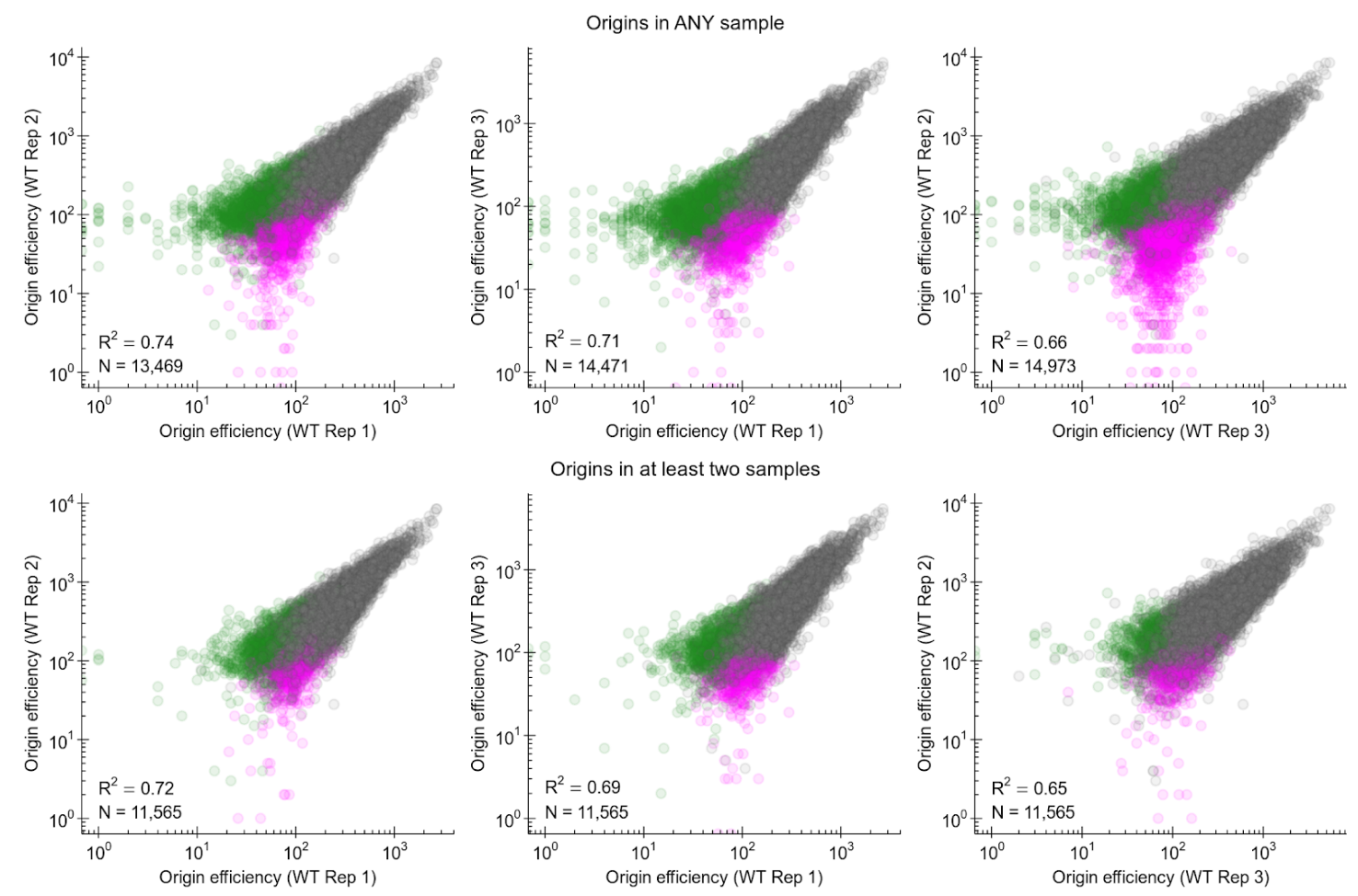

\section{Figure S5. Ori-SSDS gives a reproducible readout of origin efficiency}

618 Origin efficiency is a measure of the frequency at which each origin is used. This is inferred from

619 the sequencing read coverage in each experiment (see methods). Grey dots represent origins

620 found in both samples. Magenta and green dots represent origins found only in the $x$-axis

621 designated and y-axis designated samples, respectively. The top panels show all origins found

622 in either experiment for each pair-wise comparison. The bottom panels show only the

623 consensus set of 11,565 origins. $R^{2}$ is the squared Spearman correlation coefficient of log

624 transformed values. $\mathrm{N}$ indicates the number of origins for each pairwise comparison. 

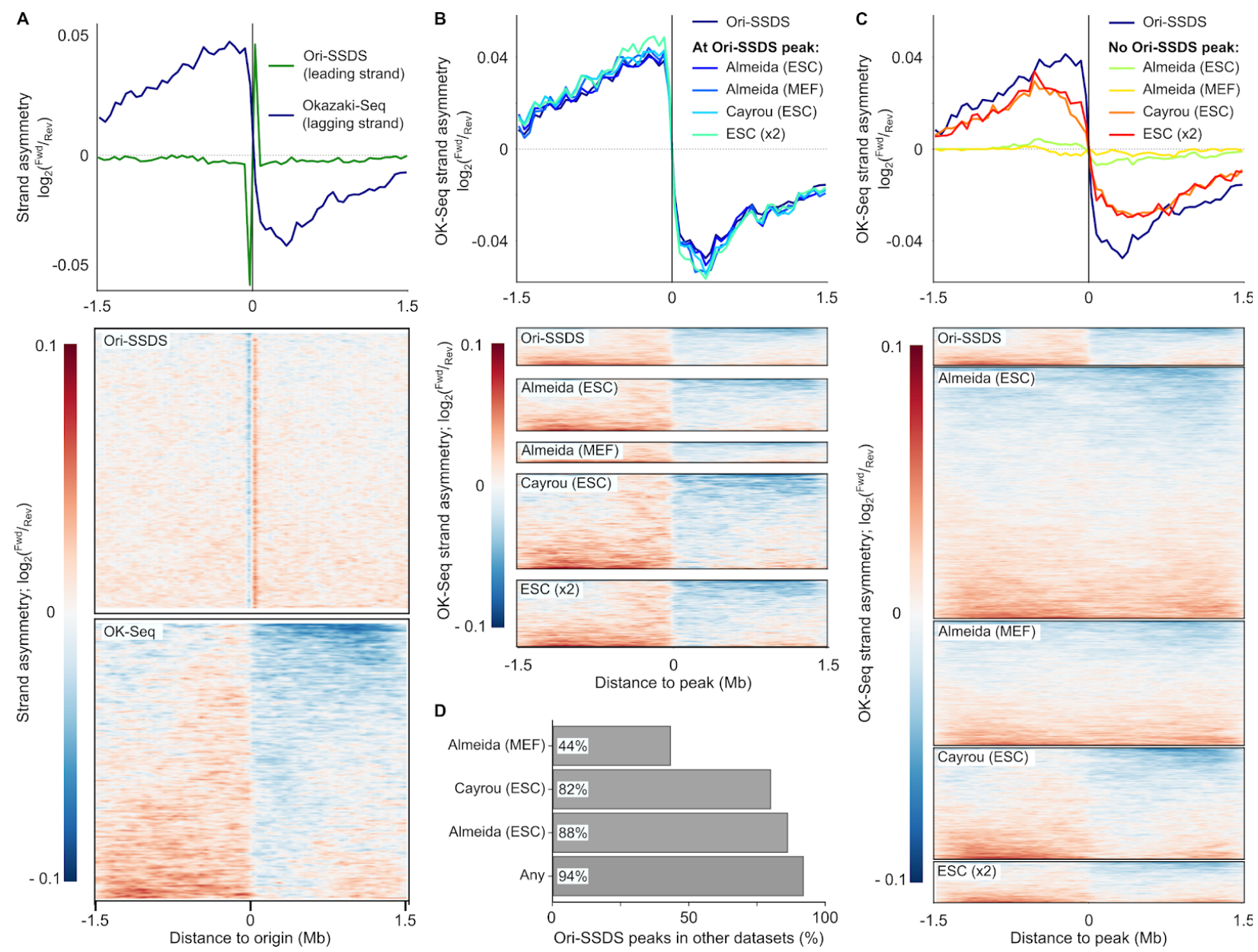

\section{Figure S6. Ori-SSDS peaks coincide with replication measures from other experiments.}

626 (A) Okazaki fragments are distributed asymetrically around Ori-SSDS-defined origins of 627 replication. Okazaki fragment sequencing (Ok-Seq) data in Embryonic Stem Cells (ESCs) are 628 from (Petryk et al., 2018). The Okazaki fragment signal is far broader than the signal from

629 Ori-SSDS because it captures all lagging-strand synthesis (for details, see (Petryk et al., 2016));

630 Ori-SSDS captures only RNA-primed leading strands at the origin of replication. Note that 631 Ok-Seq exhibits the opposite polarity to the Ori-SSDS signal as expected from capture of 632 lagging and leading strands, respectively. Data are shown in $50 \mathrm{~Kb}$ windows. Heatmaps depict 633 the signal at individual Ori-SSDS defined origins. (B,C) Ok-Seq signal at subsets of origins of 634 replication. For reference, the Ok-Seq signal at Ori-SSDS defined origins is shown in three 635 panels $(A, B, C)$. The heatmaps in panels $B$ \& $C$ are scaled to reflect the number of origins in 636 each subset. There are three genome-wide maps of origins of replication in mice. All three maps 637 were generated using SNS-Seq. One map is derived from Mouse Embryonic Fibroblast (MEF) 
638 cell culture (Almeida et al., 2018) and two from ESC culture (Almeida et al., 2018; Cayrou et al.,

639 2015). (B) Origins defined in SNS-Seq experiments that coincide with Ori-SSDS peaks exhibit

640 similar Ok-Seq asymmetry. Data for origins common to the two SNS-Seq experiments in ESCs

641 are also shown (ESC (x2)). (C) A large proportion of peaks detected in SNS-Seq experiments,

642 but not in Ori-SSDS appear to be false-positives as they lack Ok-Seq asymmetry. This is more

643 pronounced in the Almeida experiments than in the Cayrou experiment. (D) Most origins of

644 replication from Ori-SSDS in testis coincide with origins mapped in other cell types. Origins from

645 the three SNS-Seq experiments were combined to assess the overlap with "Any" origins defined

646 previously. 
bioRxiv preprint doi: https://doi.org/10.1101/2020.09.23.308874; this version posted September 23, 2020. The copyright holder for this preprint (which was not certified by peer review) is the author/funder. This article is a US Government work. It is not subject to copyright under 17 USC 105 and is also made available for use under a CCO license.
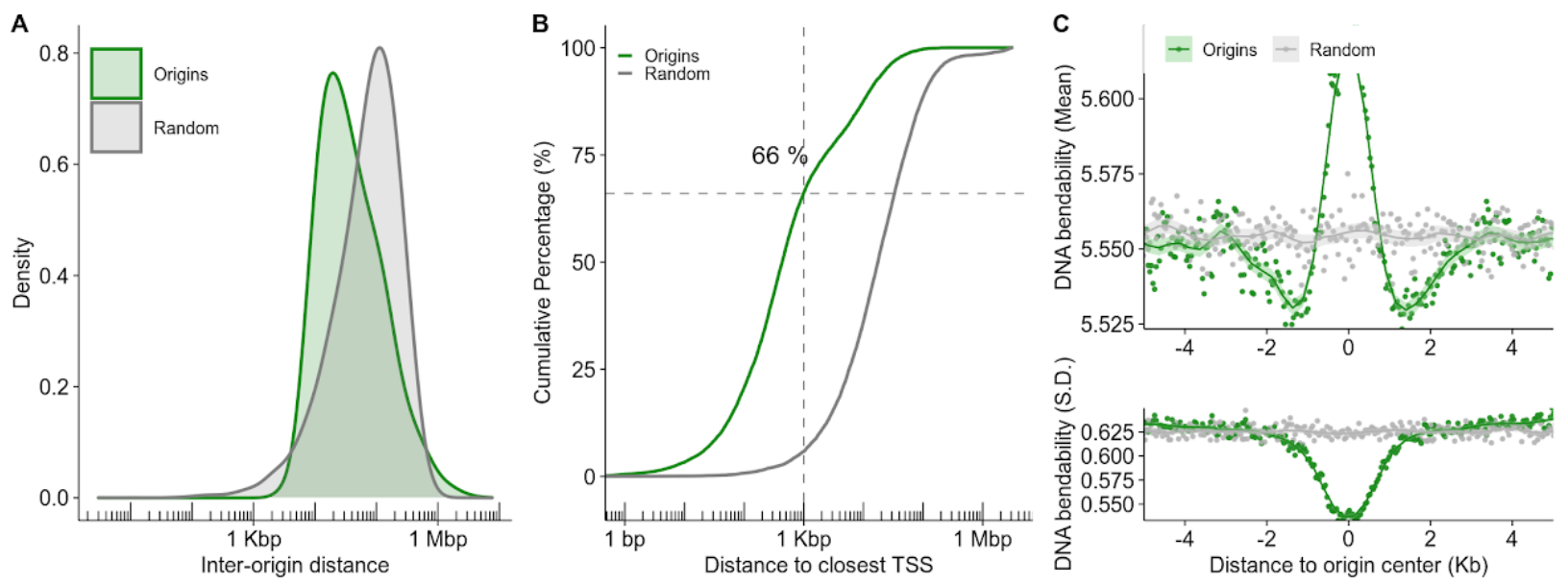

D
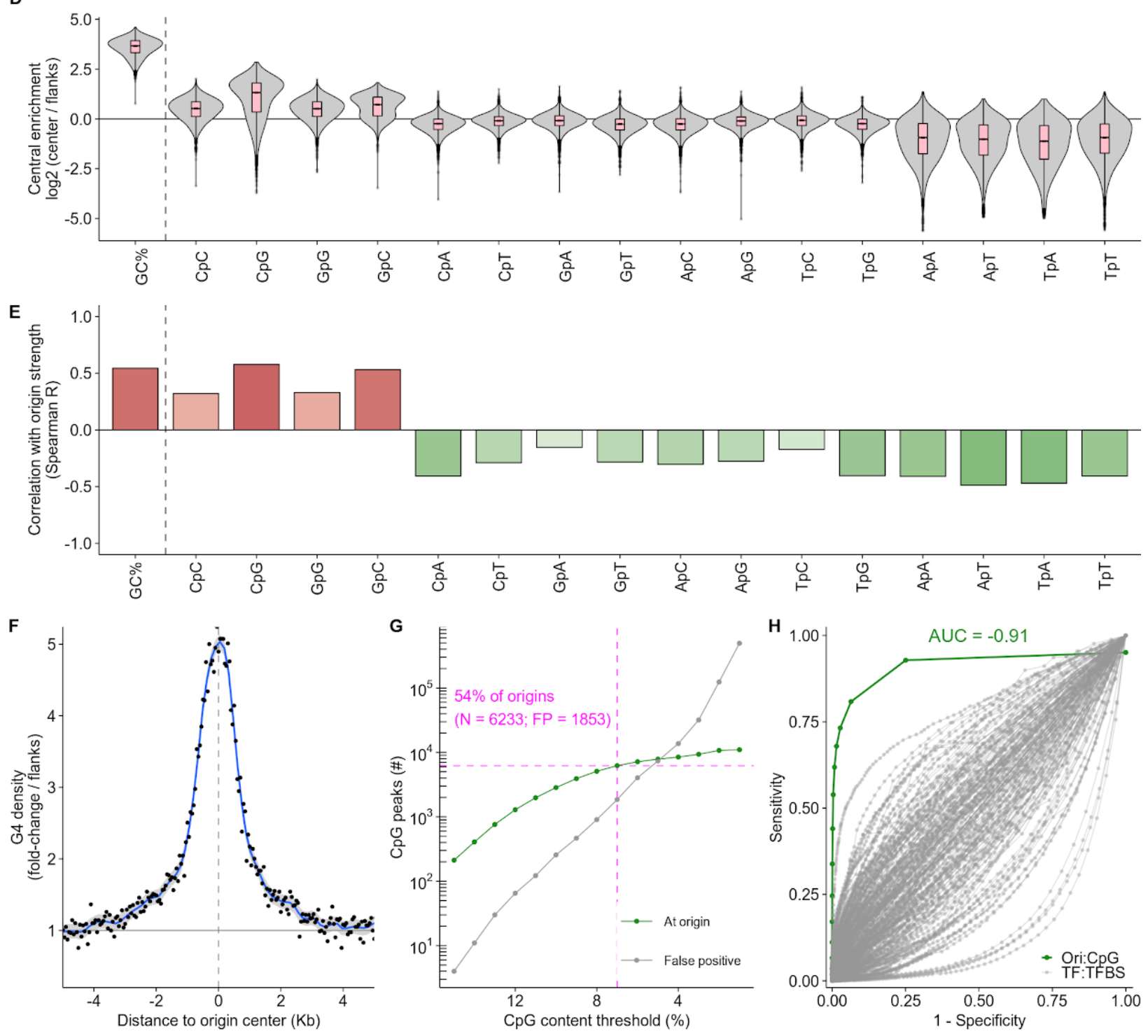
647 Figure S7. Origins of replication in mouse testis occur at GC-rich, accessible sites.

648 (A) Origins of replication are more closely spaced than expected in the genome. The expected 649 distances between randomly chosen intervals is shown in grey. (B) Most origins occur near 650 gene promoters; $66 \%$ occur within $1 \mathrm{Kbp}$ of a transcription start site (TSS), far more than 651 expected (random; grey line). (C) Replication origins coincide with local changes in DNA 652 bendability (calculated from (Goodsell and Dickerson, 1994)). The mean (top) and standard 653 deviation (bottom) of the DNA bendability at all origins is shown. Each point represents a $100 \mathrm{bp}$ 654 window. Solid line is a LOESS fit. (D) Origin centers are GC-rich and enriched in CpG and GpC 655 dinucleotides. The $\log _{2}$ ratio of the central 500 bp compared to the region flanking this center is 656 shown. (E) GC-content (GC\%), CpG density and GpC density correlate positively with origin 657 efficiency. (F) Putative G4-forming sequences are highly enriched at the center of origins. 68\% 658 of origins have a putative G4-forming sequence in the central $\pm 1 \mathrm{~Kb}$. (G) Origin locations can 659 be identified from CpG density. CpG dinucleotide density was evaluated in all $1 \mathrm{~Kb}$ regions in 660 the genome using a $100 \mathrm{bp}$ sliding window. Intervals at each CpG threshold were expanded \pm $661100 \mathrm{bp}$ around the center and overlapping windows were merged. The centerpoint of each 662 merged interval was defined as a CpG peak. CpG peaks with CpG content $\geq 7 \%$ overlap 6,233 663 origins (53\% of total). 1,853 CpG peaks at this threshold do not occur at an origin (FP). (H) 664 Origins are better predicted by CpG density than transcription factors (TFs) are by transcription 665 factor binding sites (TFBS). Receiver operating curve (ROC) for predicting origin locations using 666 CpG peaks (green) or for predicting peaks in ChIP-Seq datasets for 179 transcription factors 667 (grey). For origins, at each CpG threshold (1\% to 15\% in 1\% steps), peaks were called (as in 668 G). All mouse TFs with ChIP-Seq data (in GTRD database; (Yevshin et al., 2017)) and with a 669 designated TFBS in the HOCOMOCO database (Kulakovskiy et al., 2018) were used. 670 SPRY-SARUS (Kulakovskiy et al., 2018) was used to identify TFBS motifs genome-wide with $671 \mathrm{p}<0.005$. For each TF, the score at which $<=12,000$ peaks were found was used as a lower 672 threshold (this is to assure a fairer comparison with origins). Motifs with scores less than this 673 were discarded. TFs with $<12,000$ peaks not used. Hits inside ChIP-Seq peaks were considered 674 true positives. 
bioRxiv preprint doi: https://doi.org/10.1101/2020.09.23.308874; this version posted September 23, 2020. The copyright holder for this preprint (which was not certified by peer review) is the author/funder. This article is a US Government work. It is not subject to copyright under 17 USC 105 and is also made available for use under a CCO license.

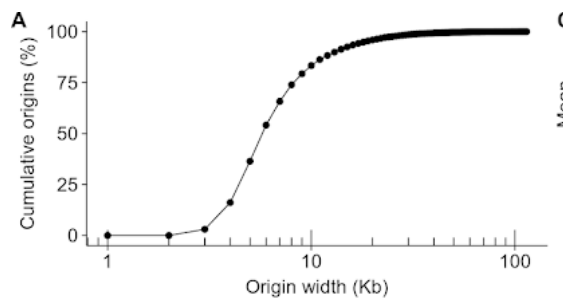

B $\quad 5,061 / 7,602(67 \%)$ narrow origins $(<6 \mathrm{~Kb})$
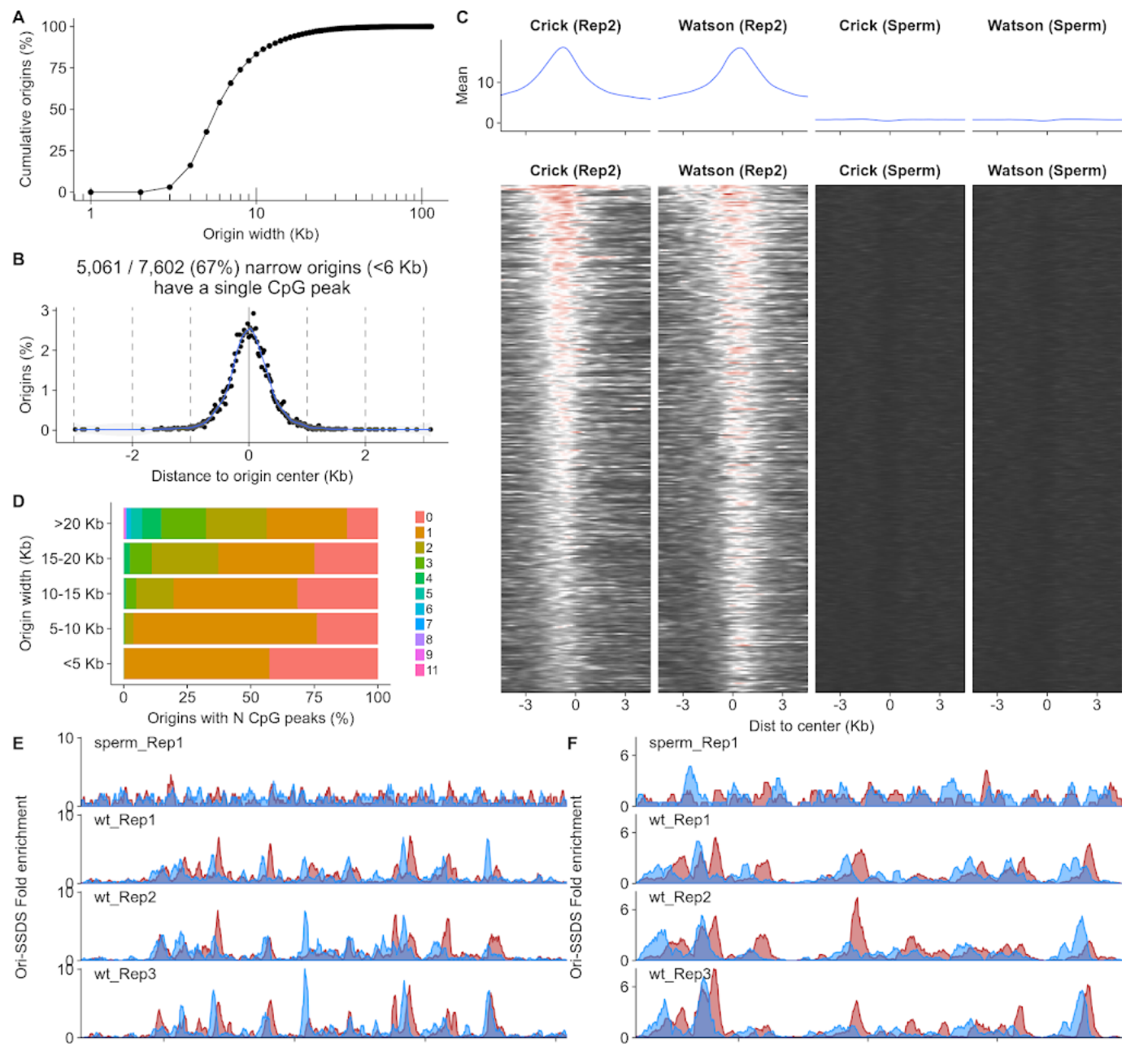

F $\quad$. sperm_Rep1
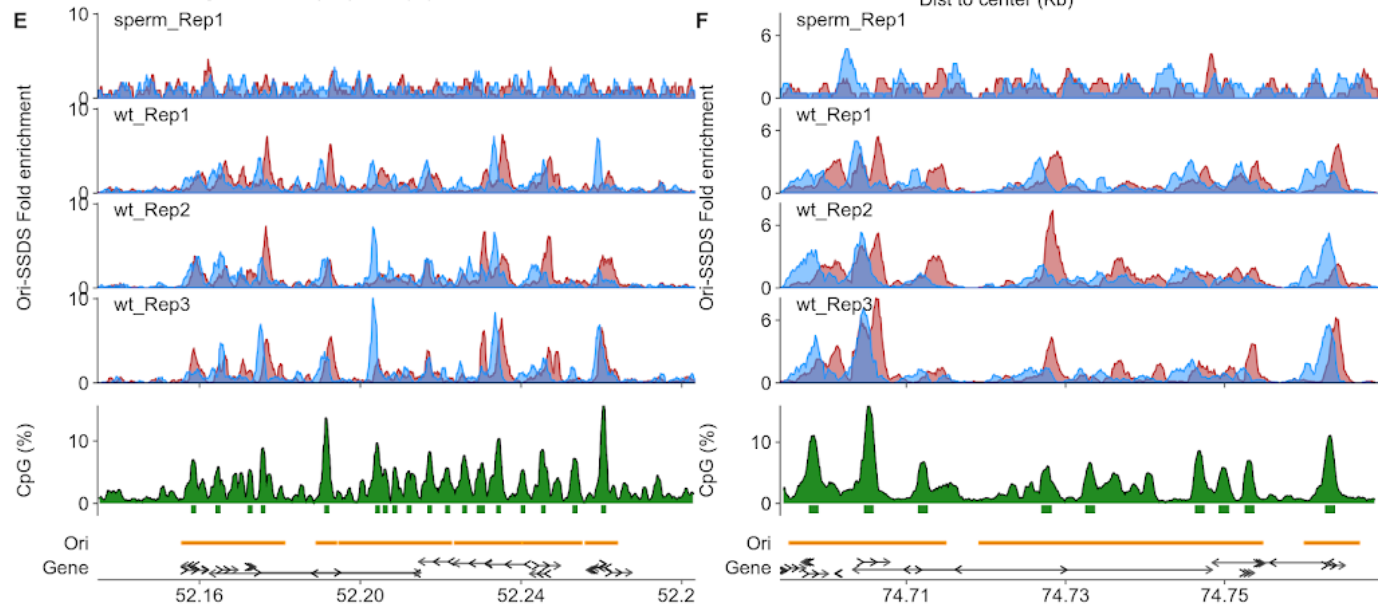

Position on chr6 (Mbp); Region width $=150 \mathrm{~Kb}$
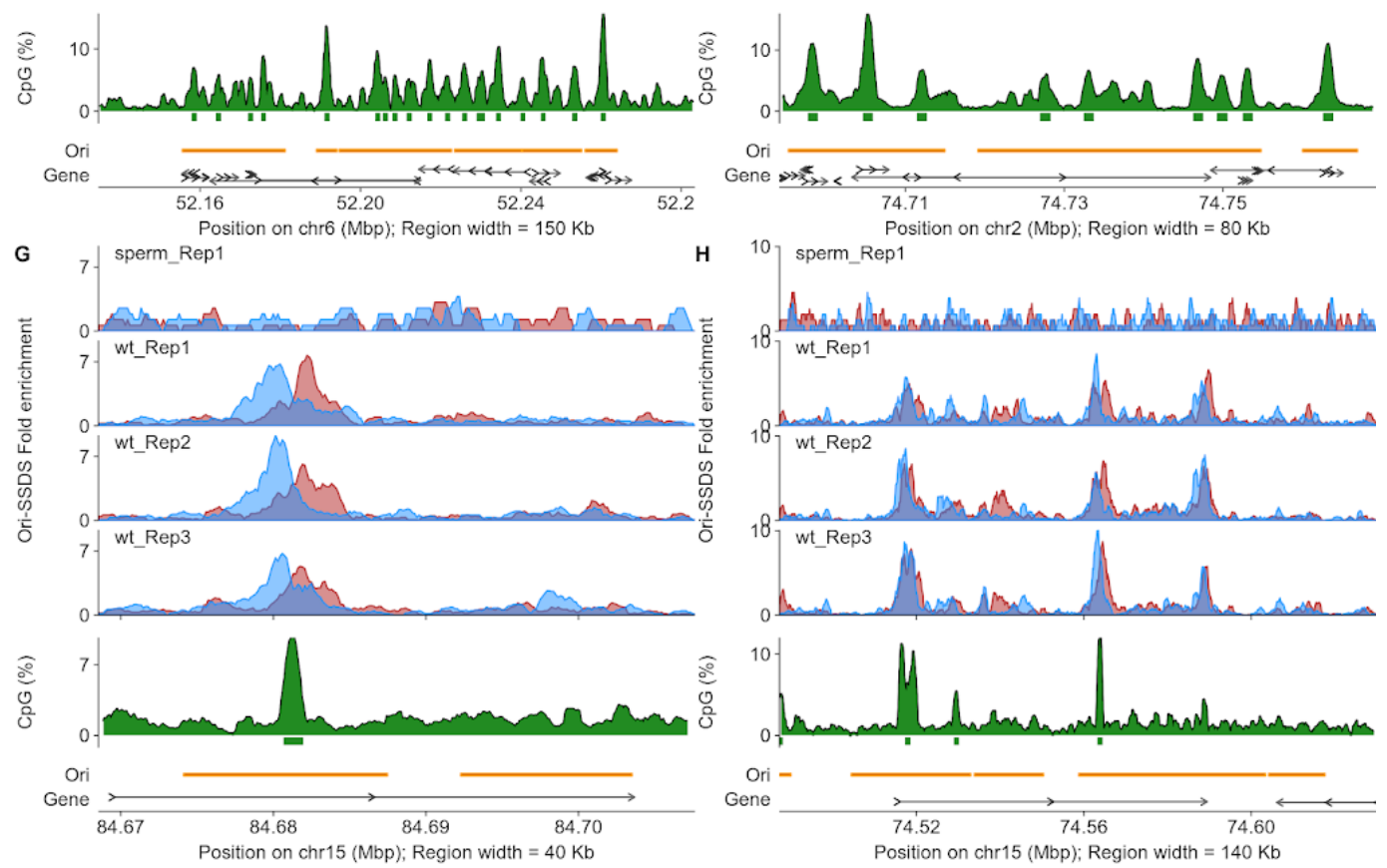

H

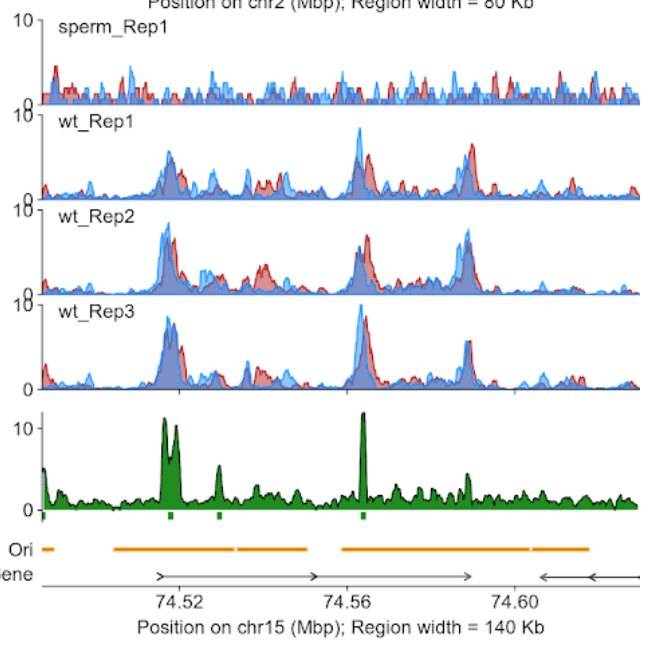




\section{Figure S8. Deconvolution of closely spaced origins of replication}

676 (A) Origin of replication cumulative width distribution. (B) Peaks of CpG density coincide with 677 origin centers. Only origins narrower than $6 \mathrm{~Kb}$ were considered. CpG dinucleotide density was

678 evaluated in all $1 \mathrm{~Kb}$ regions in the genome using a $100 \mathrm{bp}$ sliding window. Intervals with $>5 \%$

679 CpGs were expanded \pm 100 bp around the center and overlapping windows were merged. The 680 centerpoint of each merged interval was defined as a CpG "peak". (C) Almost all CpG peaks 681 within broad initiation zones (origins $>10 \mathrm{~Kb}$ ) exhibit Ori-SSDS Crick-Watson asymmetry. This 682 strongly implies that these are individual origins of replication. (D) Local CpG peak counts as a 683 function of origin width. (E,F) Examples of a cluster of initiation zones. Peaks of CpG density 684 coincide with Crick/Watson asymmetry at many loci, revealing extensive sub-structure. This 685 locus likely contains many discrete origins of replication that are difficult to separate in silico. 686 The Hox locus is shown in (E). (G) Two adjacent initiation zones with different properties. The 687 zone on the left is $13 \mathrm{~Kb}$ wide, yet contains a single, clear CpG peak that coincides with 688 Ori-SSDS asymmetry. This is likely a single replication origin. The zone on the right is also 13 $689 \mathrm{~Kb}$, but contains no discernable CpG peak or origin. This may represent a diffuse initiation zone 690 or a cluster of weak origins. (H) Four initiation zones with varying properties. 
A
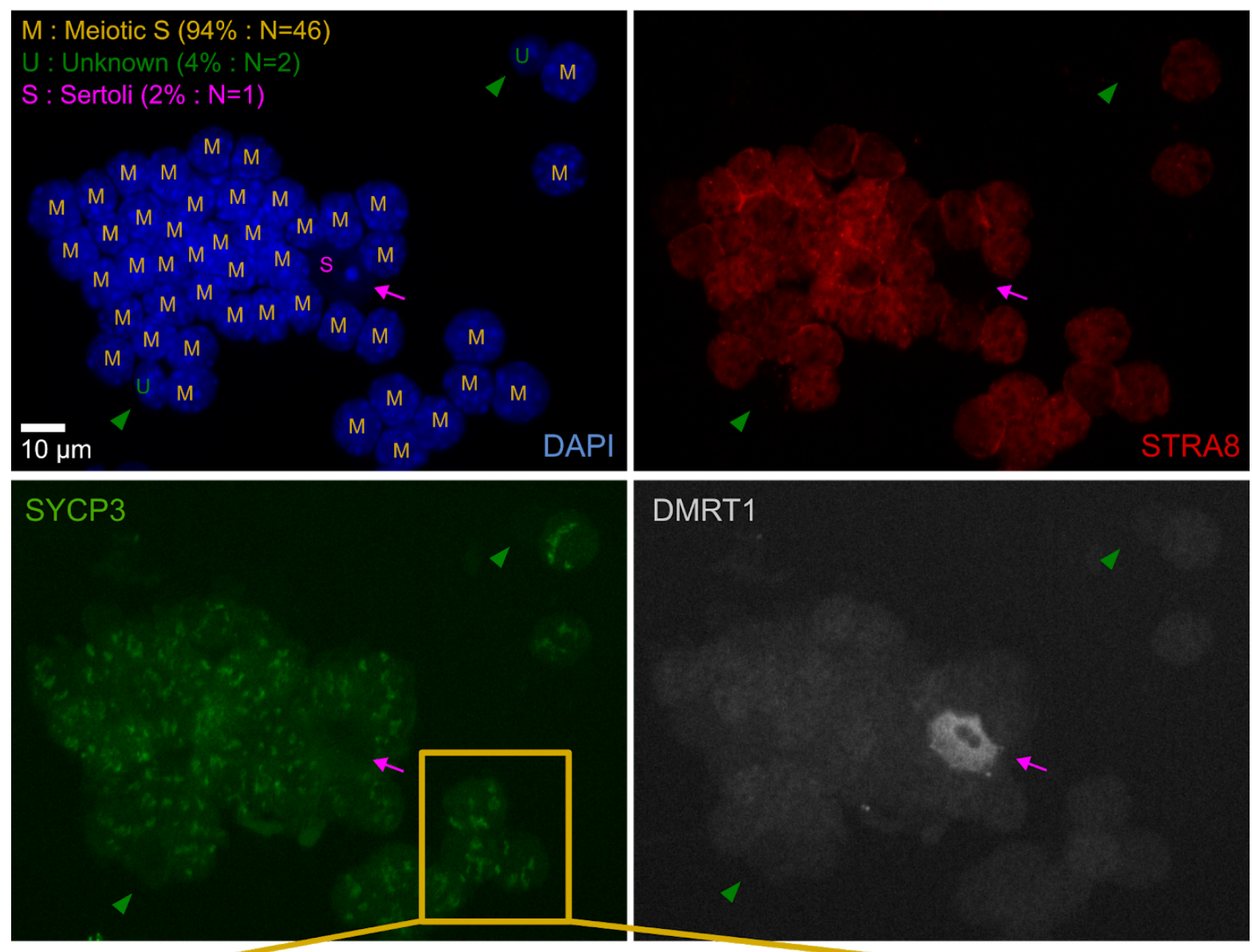

\section{DMRT1}

\section{B}

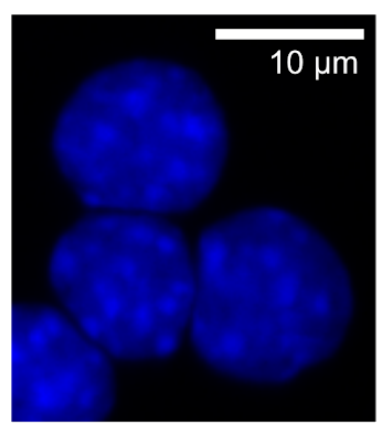

DAPI

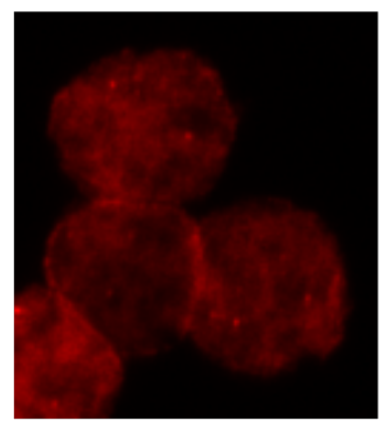

STRA8

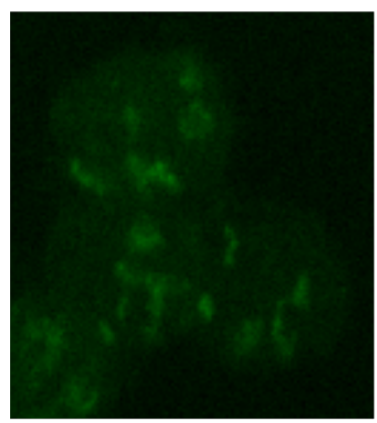

SYCP3

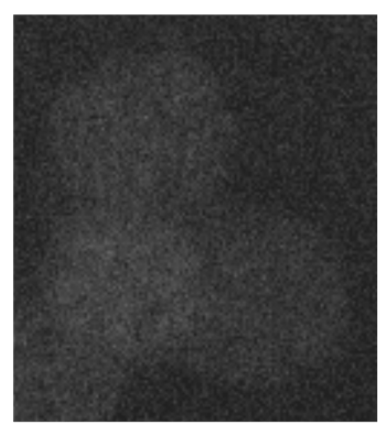

DMRT1

\section{Figure S9. High purity populations of sorted meiotic S-phase nuclei}

692 (A) Immunofluorescence microscopy images of sorted nuclei from the MeiS population (same 693 population as in Fig. 2C; 40X magnification). Nuclei were concentrated in a small volume after

694 centrifugation. The nuclei suspension was then pipetted onto silane coated slides and mounted 
695 with Vectashield. 49 nuclei are shown of which 46 are preleptotene nuclei (STRA8 positive, 696 DMRT1 negative, weak and diffuse SYCP3 signal and DAPI morphology consistent with 697 preleptotene cells (Bellve et al., 1977)). The magenta arrow points to a Sertoli cell nucleus and 698 green arrowheads point to nuclei of unknown type. Nucleus types are indicated by a letter code 699 superimposed on each cell of the DAPI-stained image (top-left). (B) Details of three 700 preleptotene nuclei at higher magnification. 

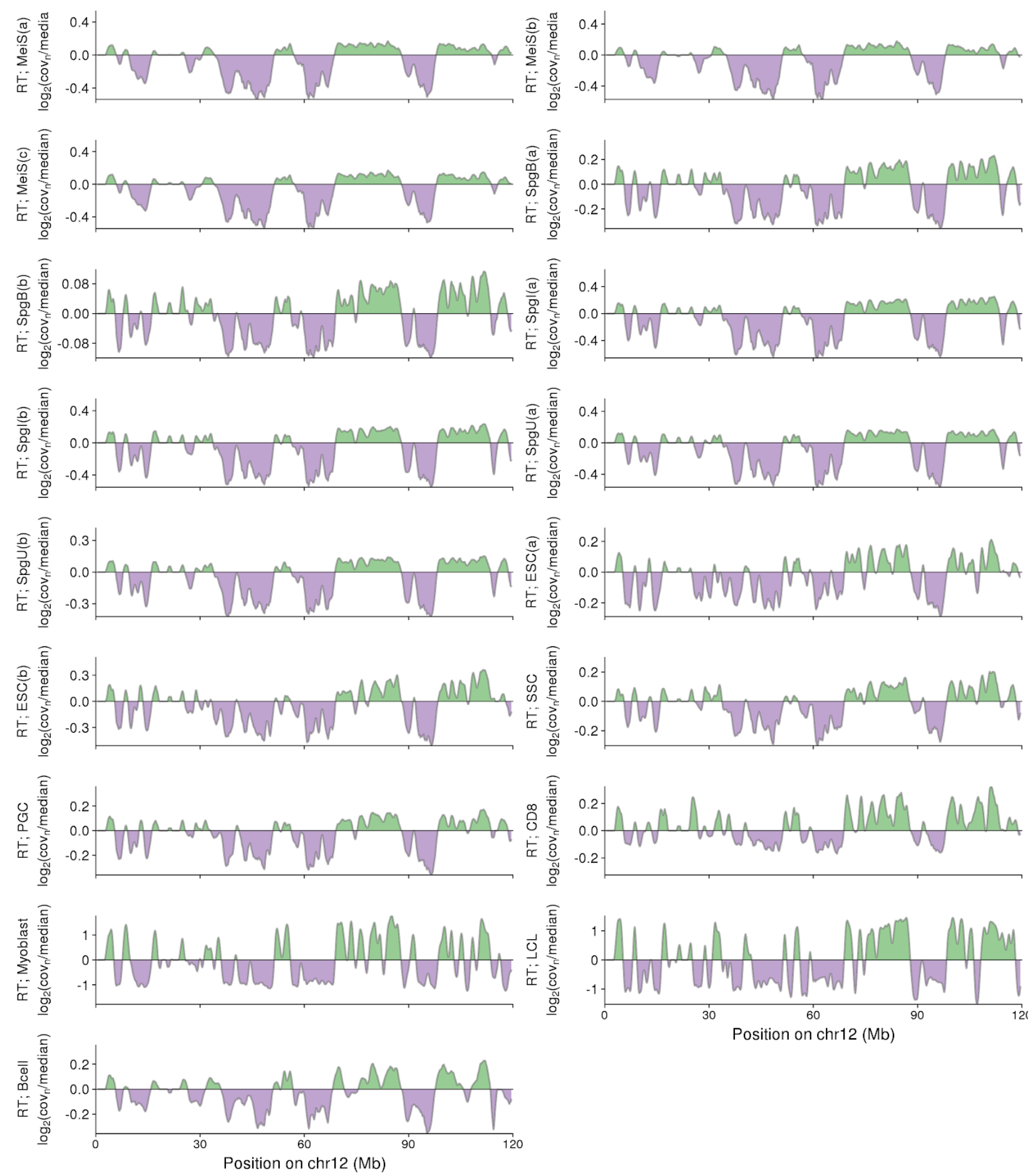

\section{Figure S10. RT-Seq yields similar RT profiles across varied cell-types}

702 RT for all cell types shown on Figure 2. RT is expressed as the $\log _{2}$ enrichment over genomic 703 median coverage for all panels except for Myoblast and LCL data. These data were obtained in 
bioRxiv preprint doi: https://doi.org/10.1101/2020.09.23.308874; this version posted September 23, 2020. The copyright holder for this preprint (which was not certified by peer review) is the author/funder. This article is a US Government work. It is not subject to copyright under 17 USC 105 and is also made available for use under a CCO license.

704 processed form from the Replication Domain database. All panels depict chromosome 12.

705 Sample names are explained briefly in Figure 3 and in detail in Tables S1-3. 


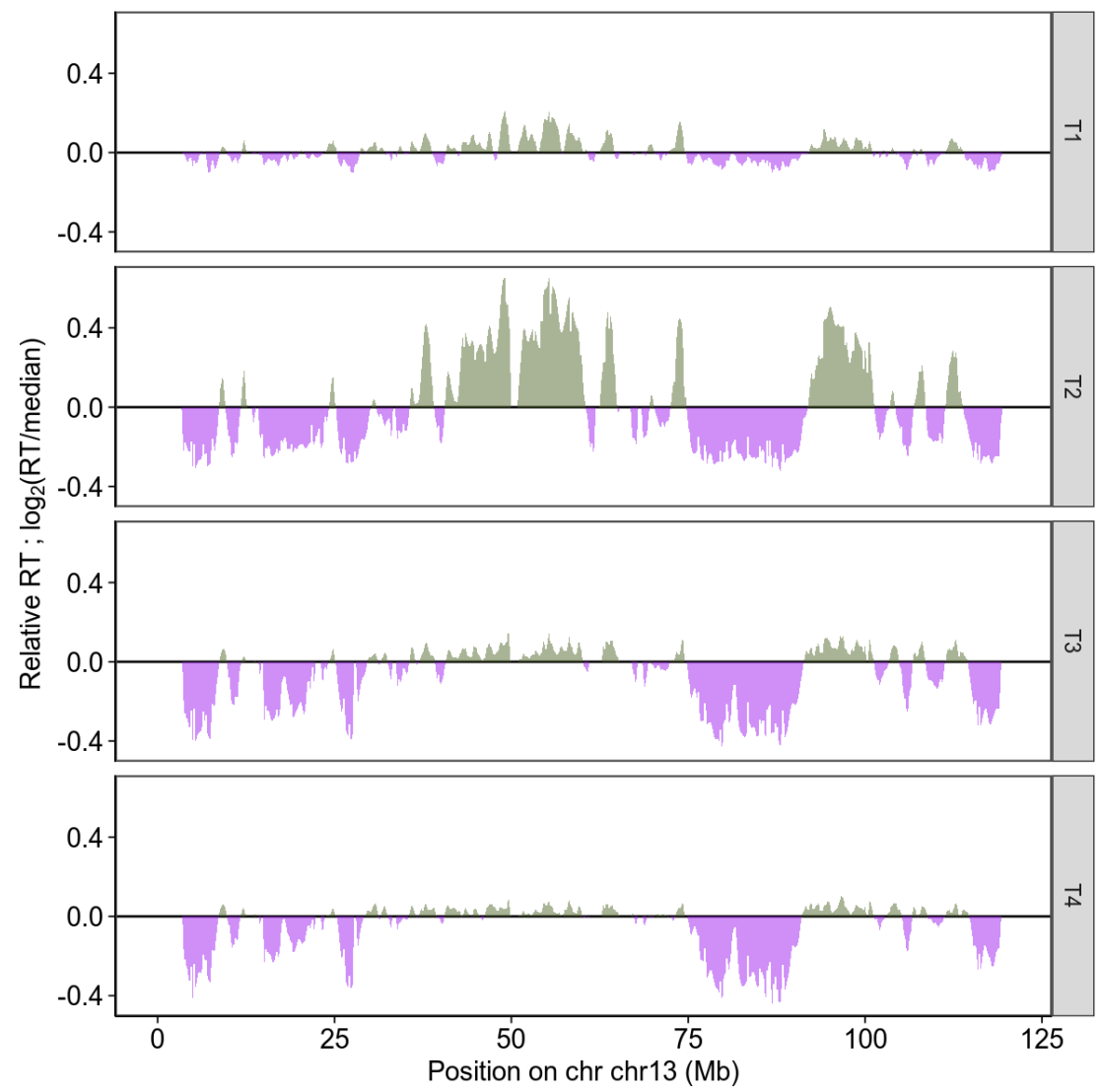

706 Figure S11. Experimentally derived RT varies as a function of the starting population

707 Four sub-populations of meiotic S-phase were isolated from early S (T1) through late S (T4). RT

708 was inferred from WGS (see methods). The earliest and latest replicating regions exhibit 709 substantial differences in RT across these four populations. 
bioRxiv preprint doi: https://doi.org/10.1101/2020.09.23.308874; this version posted September 23, 2020. The copyright holder for this preprint (which was not certified by peer review) is the author/funder. This article is a US Government work. It is not subject to copyright under 17 USC 105 and is also made available for use under a CCO license.
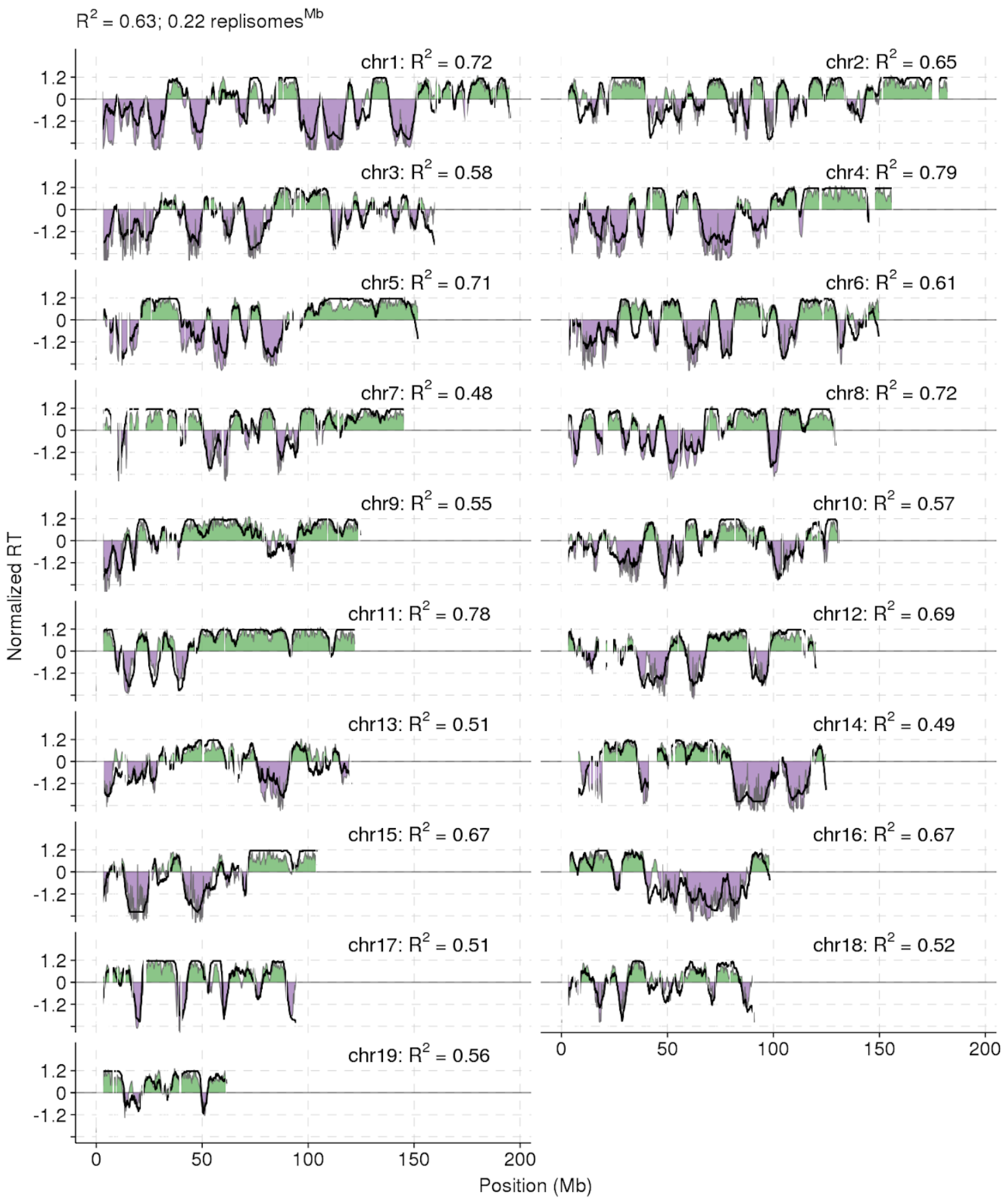
bioRxiv preprint doi: https://doi.org/10.1101/2020.09.23.308874; this version posted September 23, 2020. The copyright holder for this preprint (which was not certified by peer review) is the author/funder. This article is a US Government work. It is not subject to copyright under 17 USC 105 and is also made available for use under a CCO license.
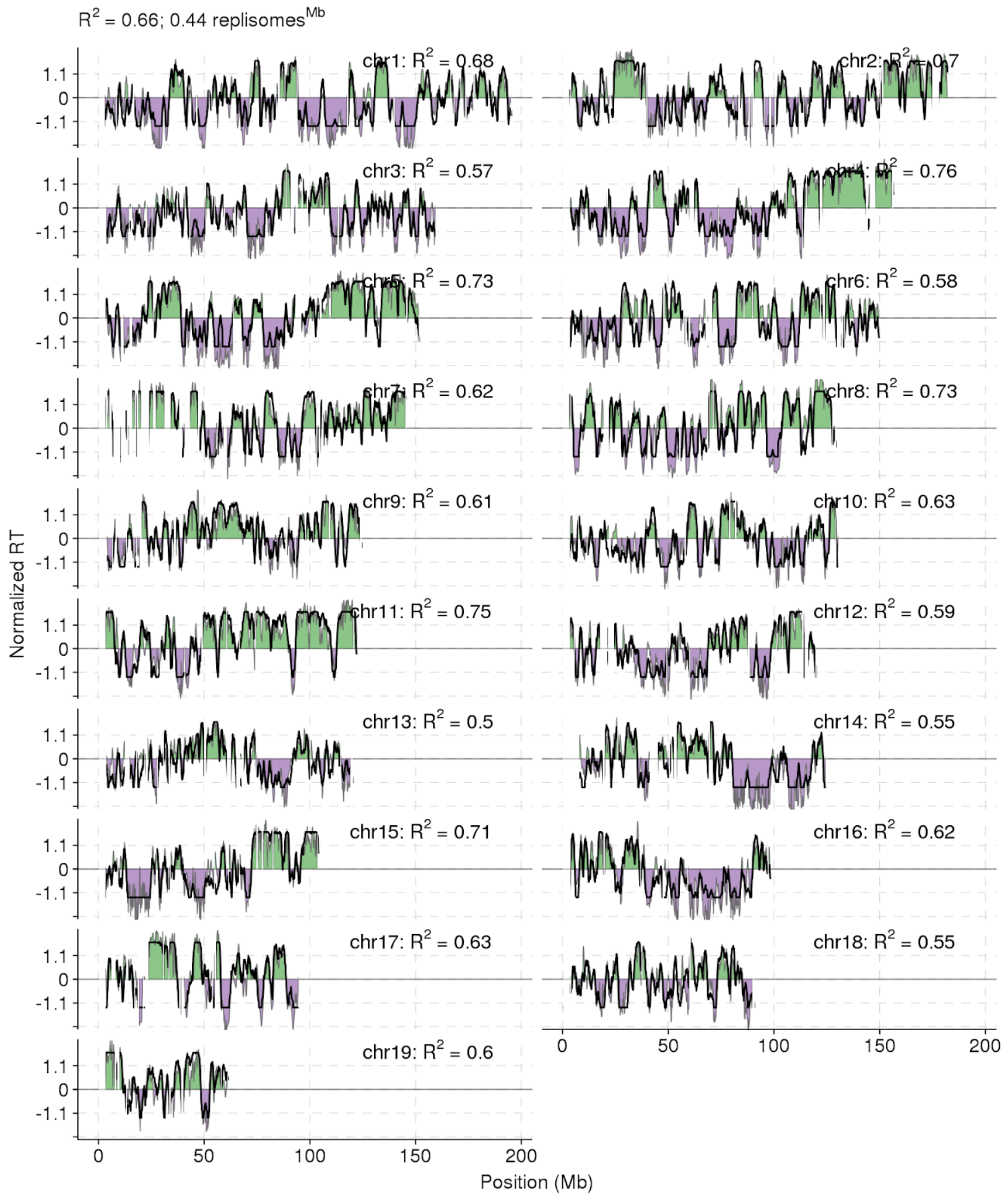


\section{Figure S12. Modelling recapitulates experimental RT genome-wide}

713 Experimental RT is shown as filled green (early replicating) and violet (late replicating) regions.

714 The best-fitting RT-Sim predicted RT is shown as a solid black line. Per-chromosome Pearson

715 correlation coefficients are shown. Both (A) Meiotic and (B) E14 ESC RT can be accurately

716 modeled in silico. Both experimental and predicted RT are normalized by mean and standard

717 deviation (s.d.); (RTnorm = (RT-mean(RT)) / s.d.(RT)). 


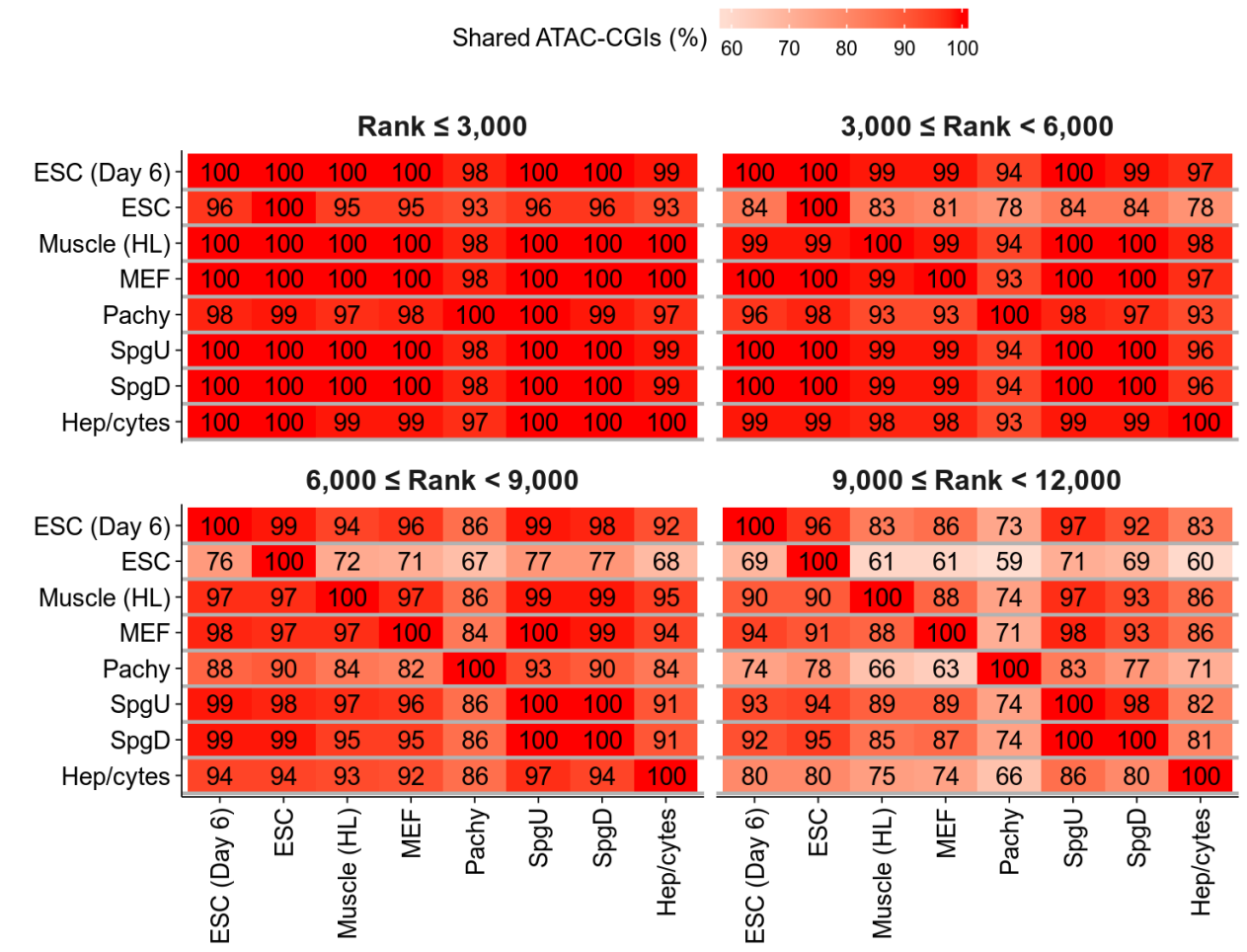

\section{Figure S13. ATAC-Seq peaks at CpG islands are broadly conserved across diverse}

719 cell-types.

720 We called peaks from 8 published ATAC-Seq experiments in a variety of mouse cell types. The

721 strongest 12,000 peaks were split into 4 groups by strength. We counted the overlap of each

722 peak subset with the top 12,000 ATAC-Seq peaks from each other cell-type. The percentage of

723 peaks from each subset (y-axis) found in other cell types (x-axis) is shown. Datasets used are

724 as follows: Hepatocytes (Hep/cytes; SRA accessions: SRR6813698,SRR6813699)(Li et al.,

725 2019b), Differentiated cKIT+ Spermatogonia (SpgD; SRA accessions: SRR5956508)(Maezawa

726 et al., 2018), Undifferentiated THY+ Spermatogonia (SpgU; SRA accessions:

727 SRR5956504)(Maezawa et al., 2018), Pachytene Spermatocytes (Pachy; SRA accessions:

728 SRR5956512)(Maezawa et al., 2018), Mouse Embryonic Fibroblasts (MEF; SRA accessions:

729 SRR7048429, SRR7048430), Hindlimb Muscle Cells E14.5 (Muscle (HL); SRA accessions:

730 SRR8104383,SRR8104391)(Castro et al., 2019), Embryonic Stem Cells (ESC; SRA

731 accessions: SRR7048437,SRR7048438), Embryonic Stem Cells - 6 Days (ESC (Day 6); SRA

732 accessions: SRR7048433,SRR7048434). 
bioRxiv preprint doi: https://doi.org/10.1101/2020.09.23.308874; this version posted September 23, 2020. The copyright holder for this preprint (which was not certified by peer review) is the author/funder. This article is a US Government work. It is not subject to copyright under 17 USC 105 and is also made available for use under a CCO license.

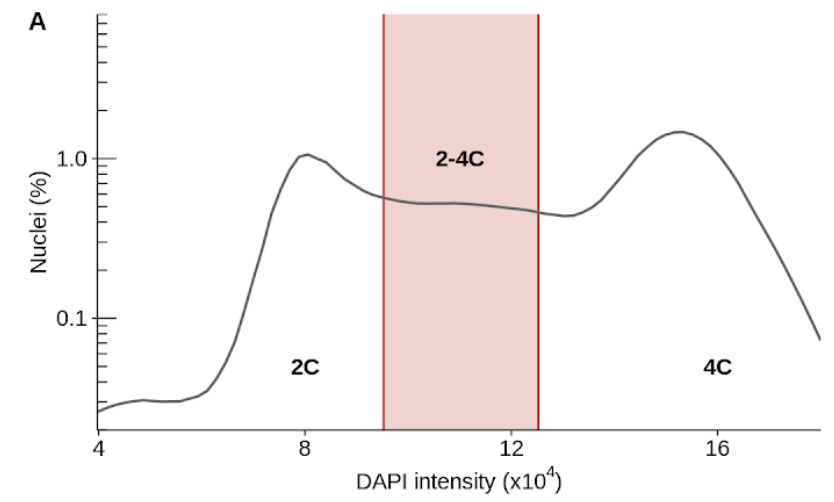

C
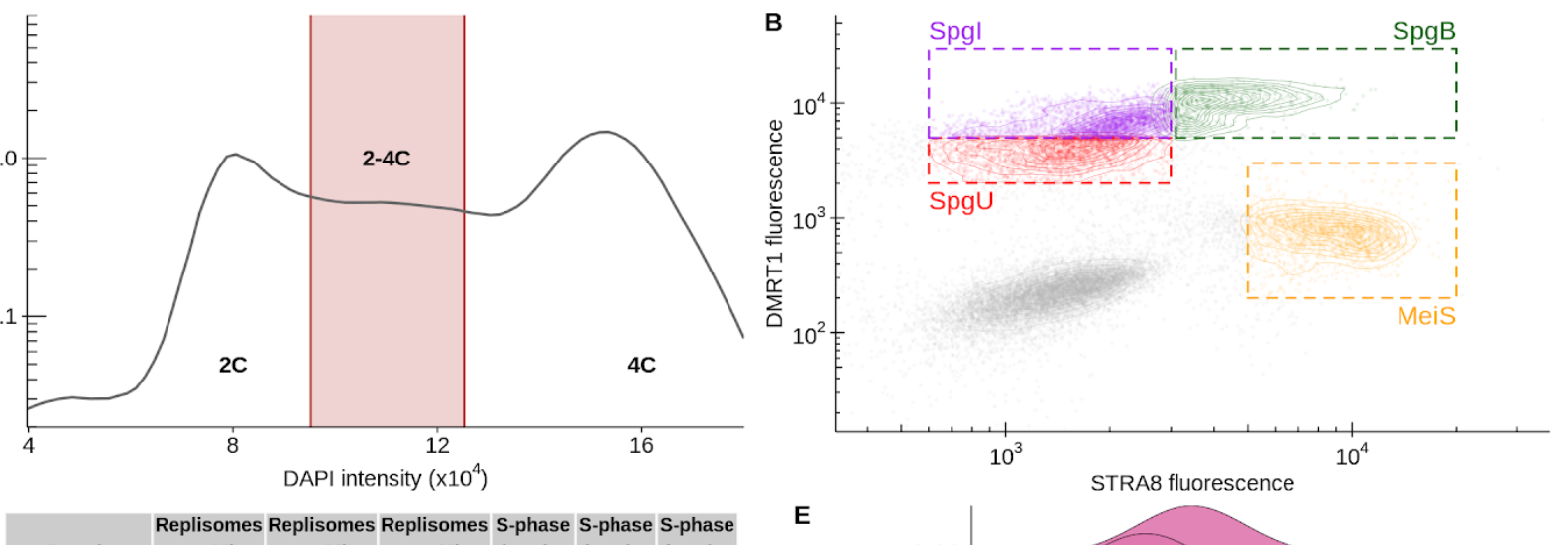

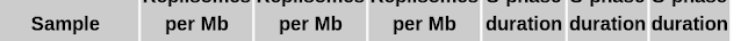
\begin{tabular}{|c|c|c|c|c|c|c|} 
& (median) & (mean) & (s.d.) & (median) & (mean) & (s.d.) \\
MeiS(SPO11) & 0.26 & 0.26 & 0.03 & 395 & 403 & 34
\end{tabular} \begin{tabular}{|c|c|c|c|c|c|c|}
\hline MeiS(wtLM) & 0.26 & 0.26 & 0.02 & 396 & 402 & 31 \\
\hline
\end{tabular} \begin{tabular}{|l|l|l|l|l|l|l|}
\hline MeiS(c) & 0.24 & 0.25 & 0.03 & 421 & 416 & 48 \\
\hline
\end{tabular} \begin{tabular}{|l|l|l|l|l|l|l|}
\hline MeiS(b) & 0.24 & 0.25 & 0.04 & 421 & 418 & 50 \\
\hline
\end{tabular} \begin{tabular}{|l|l|l|l|l|l|l|}
\hline MeiS(a) & 0.24 & 0.24 & 0.03 & 421 & 423 & 47 \\
\hline
\end{tabular} \begin{tabular}{|l|l|l|l|l|l|l|}
\hline SpgU(SPO11) & 0.42 & 0.42 & 0.04 & 271 & 273 & 18 \\
\hline
\end{tabular} \begin{tabular}{|l|l|l|l|l|l|l|}
\hline $\mathrm{SpgU}($ wtLM) & 0.40 & 0.40 & 0.03 & 281 & 285 & 17 \\
\hline
\end{tabular} \begin{tabular}{|l|l|l|l|l|l|l|}
\hline $\mathrm{SpgU}(\mathrm{b})$ & 0.38 & 0.38 & 0.05 & 291 & 295 & 29 \\
\hline
\end{tabular} \begin{tabular}{l|l|l|l|l|l|l|}
\hline $\mathrm{SpgU}(\mathrm{a})$ & 0.40 & 0.39 & 0.06 & 281 & 291 & 32 \\
\hline
\end{tabular} \begin{tabular}{|l|l|l|l|l|l|l|}
\hline Spgl(SPO11) & 0.46 & 0.46 & 0.05 & 255 & 255 & 17 \\
\hline
\end{tabular} \begin{tabular}{|l|l|l|l|l|l|l|}
\hline Spgl(b) & 0.44 & 0.44 & 0.06 & 262 & 265 & 26 \\
\hline
\end{tabular}

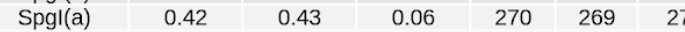
\begin{tabular}{|l|l|l|l|l|l|l|}
\hline $\mathrm{SpgB}(\mathrm{b})$ & 0.50 & 0.50 & 0.09 & 240 & 245 & 28 \\
\hline
\end{tabular} $\mathrm{SpgB}(\mathrm{a}) \quad 0.48-0.49$ \begin{tabular}{|l|l|l|l|l|l|l|}
\hline PGC & 0.46 & 0.48 & 0.08 & 254 & 253 & 28 \\
\hline
\end{tabular} \begin{tabular}{|l|l|l|l|l|l|l|}
\hline SSC & 0.42 & 0.41 & 0.07 & 271 & 279 & 33 \\
\hline
\end{tabular} \begin{tabular}{|l|l|l|l|l|l|l|}
\hline ESC(a) & 0.48 & 0.49 & 0.09 & 247 & 251 & 30 \\
\hline
\end{tabular} \begin{tabular}{|l|l|l|l|l|l|l|} 
Bcell & 0.28 & 0.29 & 0.05 & 370 & 364 & 47 \\
\hline
\end{tabular} \begin{tabular}{|l|l|l|l|l|l|l|}
\hline LCL & 0.58 & 0.60 & 0.12 & 218 & 221 & 27 \\
\hline
\end{tabular} \begin{tabular}{|l|l|l|l|l|l|l|}
\hline Myoblast & 0.58 & 0.60 & 0.11 & 219 & 220 & 25 \\
\hline
\end{tabular}

D MeiS(a) - \begin{tabular}{llll}
0.52 & 0.53 & 0.11 & 235 \\
\hline
\end{tabular}

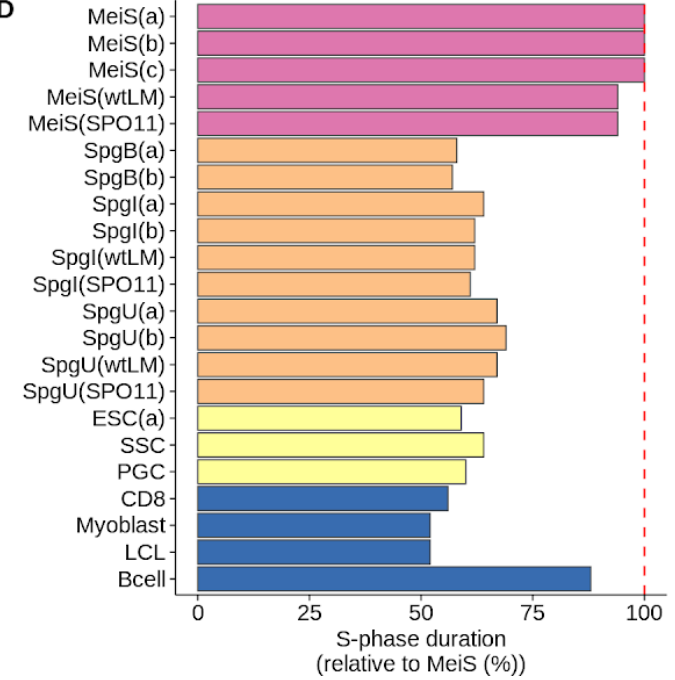

$E$

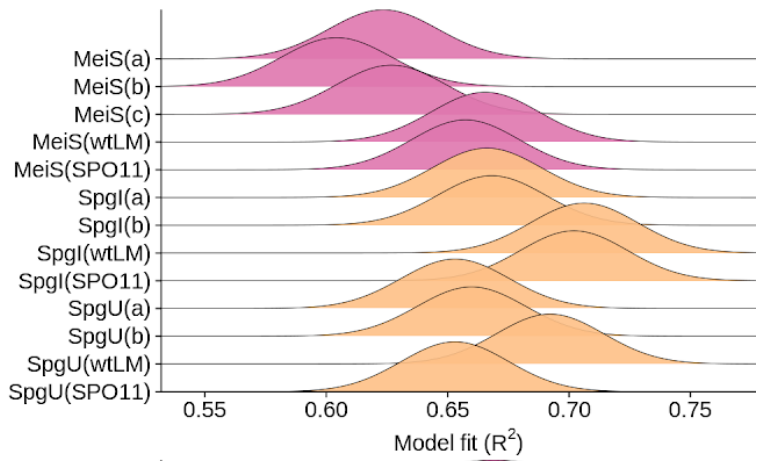

$\mathbf{F}$

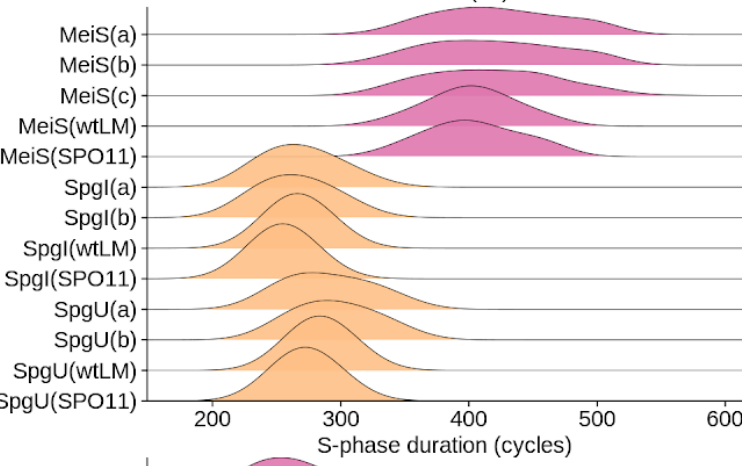

G

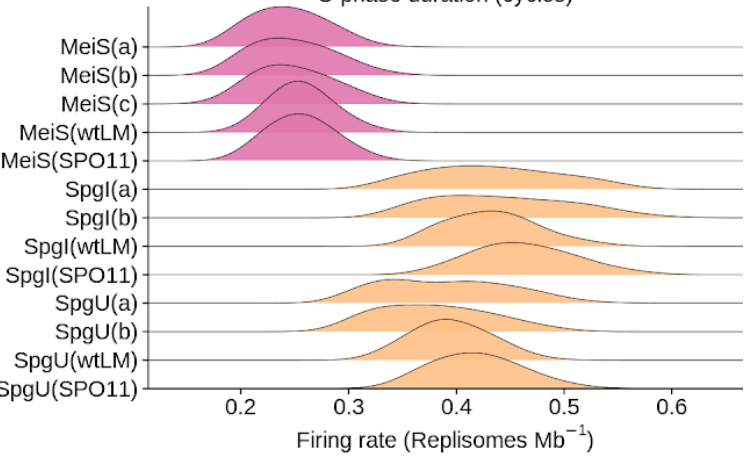

\section{Figure S14. Meiotic S-phase duration is unaffected in Spo11/\% male mice}

734 Meiotic S-phase nuclei, B-type spermatogonia and undifferentiated spermatogonia were 735 isolated from a Spo11/ male mouse testis (SPO11 in sample names) and a wild-type littermate 736 (wtLM in sample names). Details for each sample are in Tables S1-3. (A) 2-4C nuclei were 
737 sorted and (B) gated by DMRT1 and STRA8 immunofluorescence signal. Gates were defined

738 while sorting nuclei from the Spo11/- mouse and retained, unchanged for sorting nuclei from the

739 littermate. (C) Summary statistics for best-fitting RT-Sim models for all samples. (D) Comparison

740 of S-phase duration across cell types. The median S-phase duration of good models for each

741 cell-type is compared to meiotic S-phase. (E) The "fit" of RT-Sim models for Spo11/ samples is

742 comparable to the fit in other cell types. (F) The duration of meiotic S-phase is unaffected in

$743 \mathrm{Spo}^{-1}$ mice relative to the wild-type littermate control. Notably, both mice exhibit slightly

744 shorter S-phase duration compared to the three wild-type replicates. This is likely because for

745 the Spo11\% and wild-type littermate, the sorting gates were defined in the Spo $11^{-\%}$ mouse and

746 left unchanged when sorting nuclei from the littermate. Cellularity differences from the loss of

747 Spo11 may result in the capture of a slightly different population than when sorting gates are

748 defined using wild-type. (G) The firing rate is similarly unaffected. 

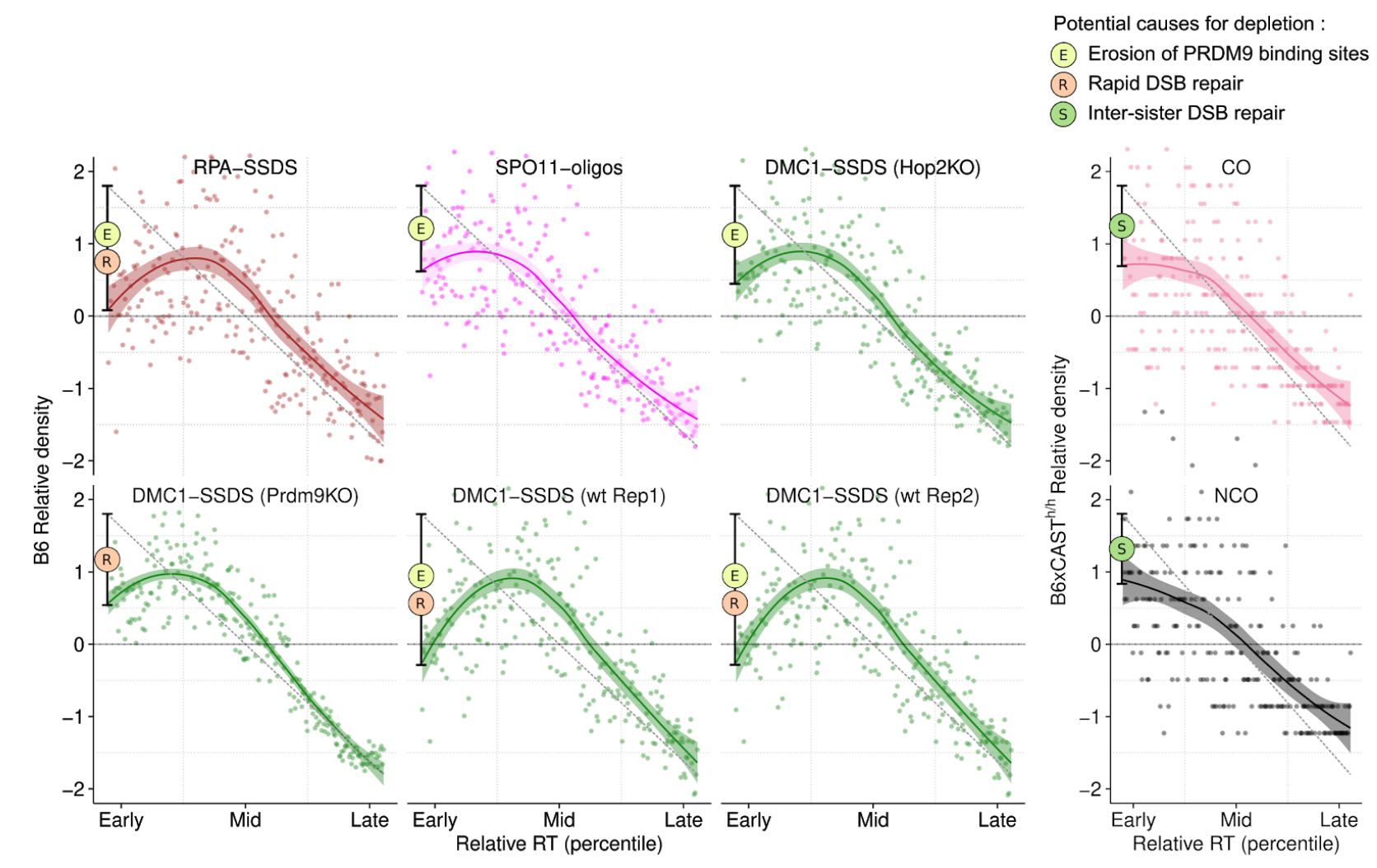

749 Figure S15. Rapid repair of DSBs in early replicating regions

750 SSDS using DMC1 exhibits a dip in the earliest replicating DNA. This is mirrored by SSDS using 751 another ssDNA-binding protein, RPA. Thus, this dip does not simply reflect a property of DMC1

752 binding (two replicates shown). HOP2 is required for strand invasion and DSB repair. In Hop2 ${ }^{-/-}$

753 mice, DSBs are not repaired and therefore any effects resulting from repair dynamics should be

754 absent. We find that the dip in the DMC1-SSDS signal in the earliest replicating DNA is

755 abolished in $\mathrm{Hop}^{-/}$mice and that the DMC1-SSDS curve now only reflects the rate of DSB

756 formation (like SPO11-oligo mapping; pink). Importantly, in mice lacking functional PRDM9, the

757 DMC1-SSDS signal also dips at the earliest replicating DNA. PRDM9 binding site erosion does

758 not affect PRDM9-independent DSB hotspots, therefore the dip is not as deep as in wild-type

759 mice, where both erosion and rapid repair play a role. Both crossovers (COs) and

760 Non-crossovers (NCO) are depleted in the earliest replicating DNA. For all figures, dots

761 represent the average signal from all autosomal bins for each RT quantile $(N=250)$. Simulated

762 RT from the T1 Meiocyte population (B6; Figure 3D) or simulated RT from a B6xCAST F1 hybrid

763 (B6xCAST) is used for RT estimates. The solid lines depict the LOESS smoothed signal \pm 
bioRxiv preprint doi: https://doi.org/10.1101/2020.09.23.308874; this version posted September 23, 2020. The copyright holder for this preprint (which was not certified by peer review) is the author/funder. This article is a US Government work. It is not subject to copyright under 17 USC 105 and is also made available for use under a CCO license.

764 standard error (shaded). The dashed grey line is a projected linear correlation and the deviation

765 from a linear correlation was manually added as a black range bar. The phenomena contributing

766 to each dip are indicated by the colored circles (see key at top). 
bioRxiv preprint doi: https://doi.org/10.1101/2020.09.23.308874; this version posted September 23, 2020. The copyright holder for this preprint (which was not certified by peer review) is the author/funder. This article is a US Government work. It is not subject to copyright under 17 USC 105 and is also made available for use under a CCO license.

A
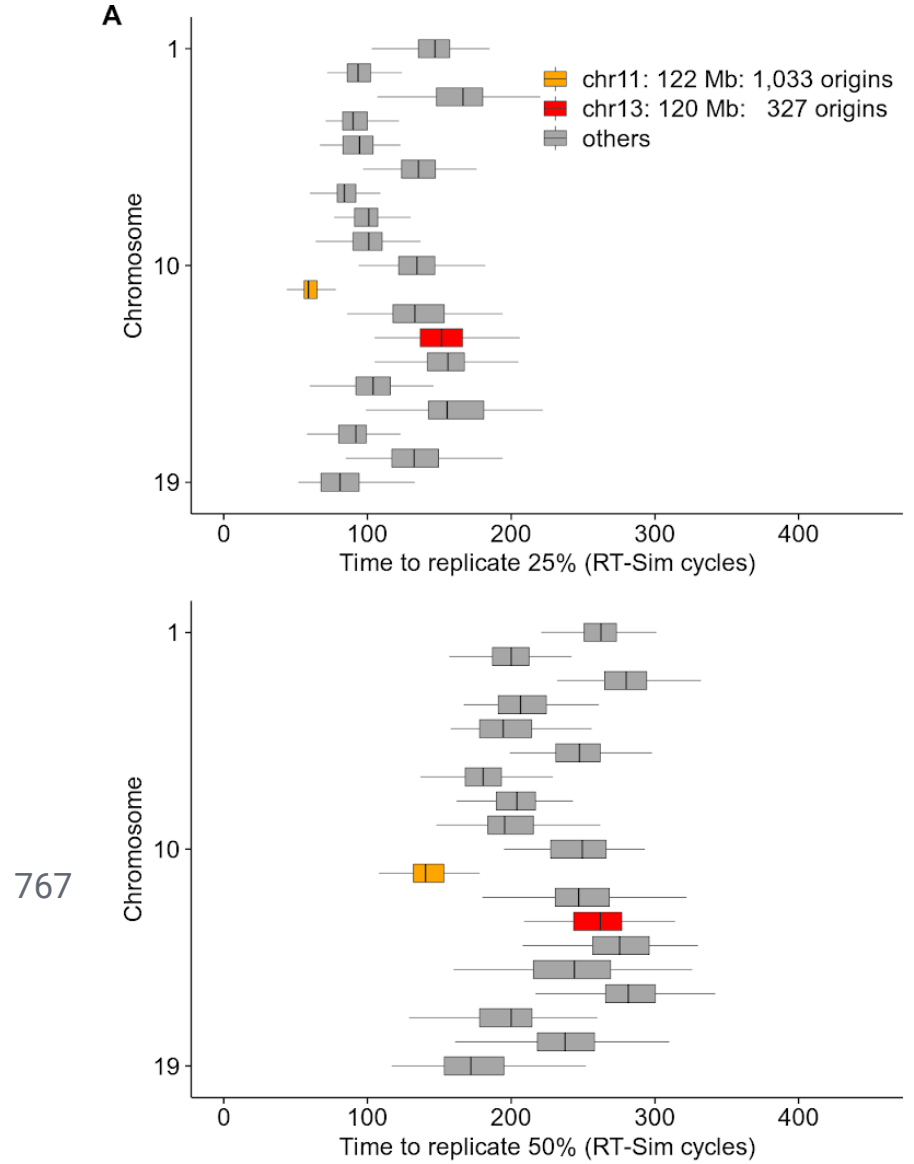

B Chr. size (poly) -

DSB hotspots (per Mb)

Rep. Speed $(75 \%)$

Chr. size (linear)

Origins (per Mb)

Rep. Speed $(50 \%)$

Rep. Speed (90\%)

Rep. Speed (25\%)

Rep. Speed (10\%)

GC (\%)

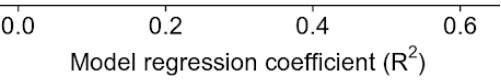

C

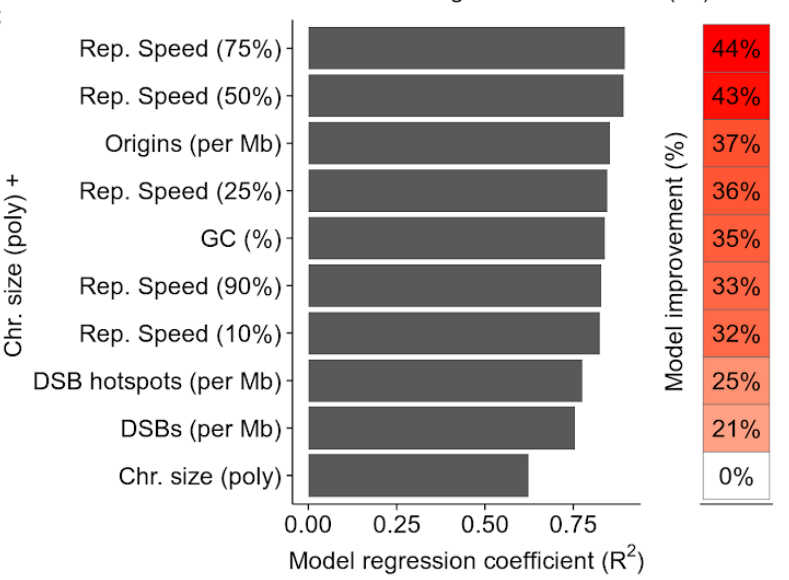

D

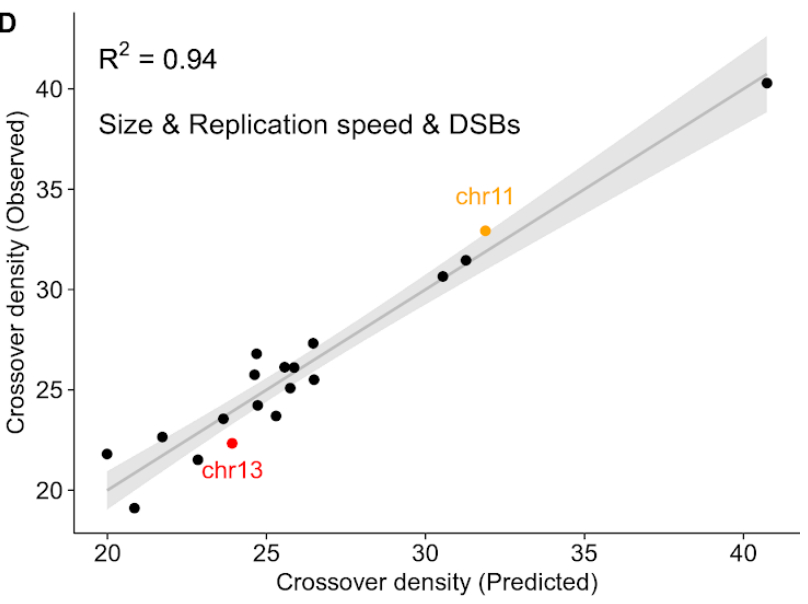

\section{Figure S16. Replication speed differs among chromosomes and can predict crossover}

\section{9 density.}

770 (A) Some chromosomes replicate faster than others; for example, chromosome 11 and 13 are a 771 similar size but chromosome 11 replicates far earlier. Notably, chromosome 11 has over 3-times 
772 more replication origins. The time required to replicate $25 \%$ (top), 50\% (middle) or 75\% (bottom)

773 of each chromosome is inferred from the best-fitting RT-Sim model. Boxplots indicate the range

774 of times from 100 individual modelled cells. The median value for each chromosome is used as

775 a measure of replication speed in subsequent panels. (B) We built simple regression models to

776 predict crossover density from each individual property of chromosomes. DSBs are derived from

777 DMC1-SSDS experiments in B6xCAST F1 mice (Smagulova et al., 2016). Crossovers in

778 B6xCAST are taken from (Yin et al., 2019) and crossover density is calculated as total

779 crossovers per Mb. Per-chromosome crossover density is best predicted by chromosome size

780 (polynomial fit). This mirrors findings in other organisms (Murakami et al., 2020). (C) Next, we

781 built a series of linear models of crossover density using chromosome size and each of the

782 other chromosome properties. Replication speed and chromosome size together are a strong

783 predictor of per-chromosome crossover density. The best combination (size + Rep.speed

$784(75 \%)$ ) yielded a $44 \%$ improvement on a model that used size alone and explained $90 \%$ of the

785 variance in per-chromosome crossover density. Origin density and GC-content also improved

786 the size-only models, but not as much as replication speed. (D) $94 \%$ of the variance in

787 per-chromosome crossover density can be explained by adding DSB density to the best model

788 from (C). 

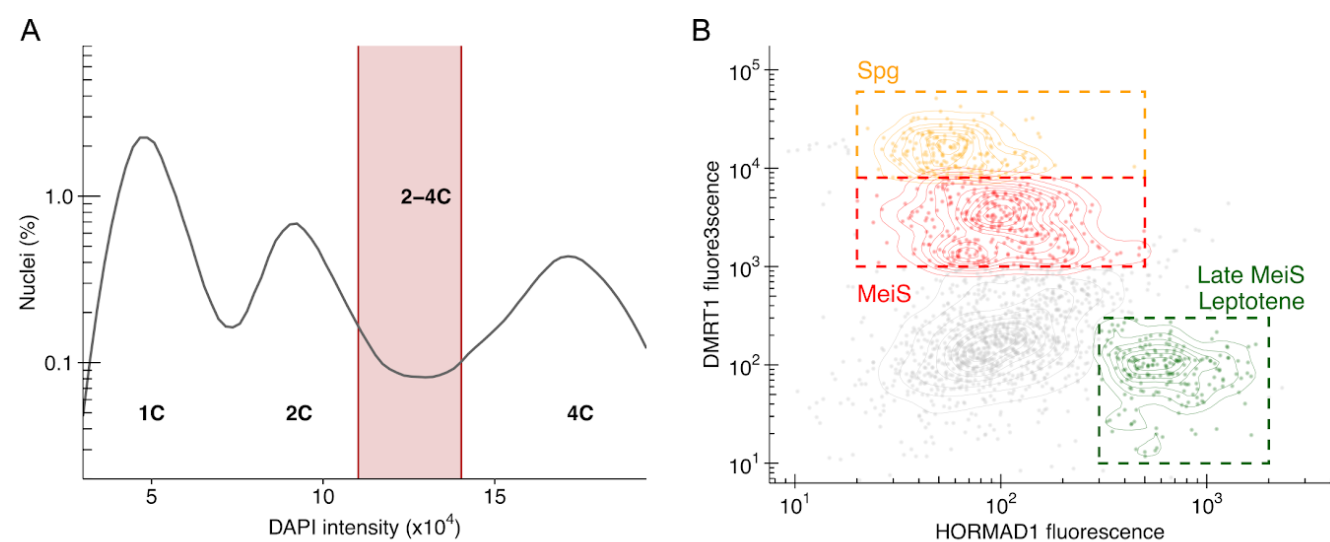

C

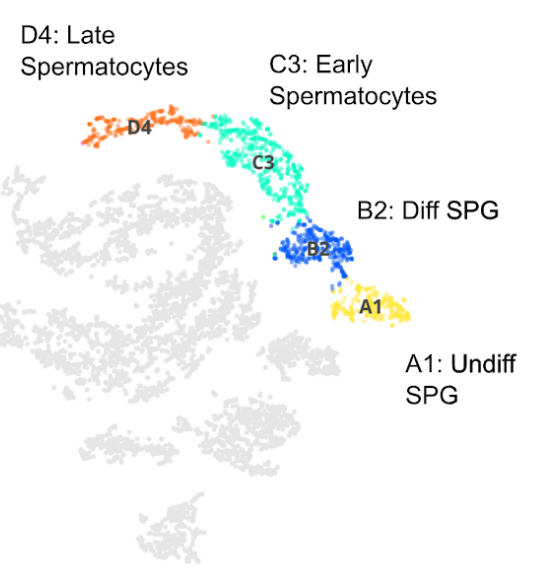

D

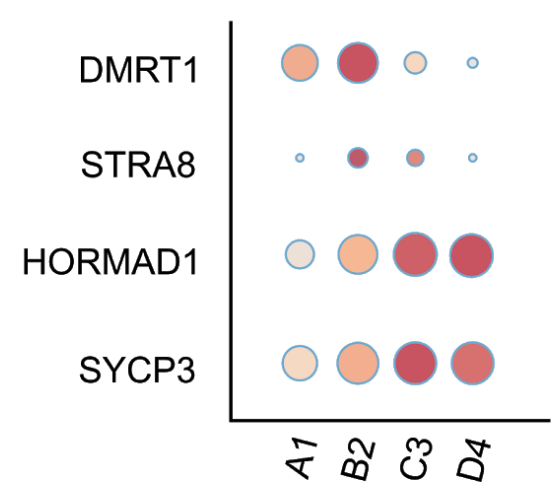

E

STRA8

DMRT1

HORMAD1

SYCP3

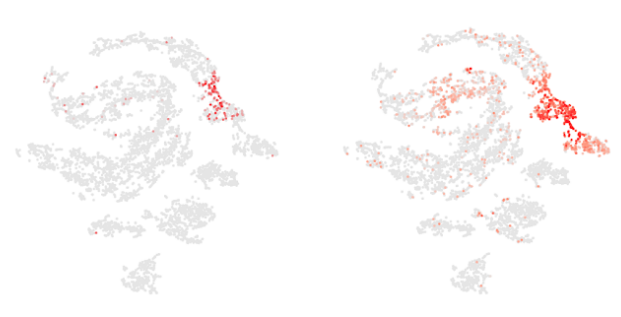

\section{Figure S17. Isolation of human meiotic S-phase nuclei}

790 (A-B) Human meiotic S-phase and spermatogonia nuclei are isolated using

791 fluorescence-activated nuclei sorting (Lam et al., 2019). (A) Replicating nuclei were isolated by

792 gating 2-4C nuclei by DAPI content. (B) Putative meiotic nuclei were isolated using a

793 combination of DMRT1 and HORMAD1. Antibodies to the STRA8 protein (used for sorting

794 mouse meiotic S-phase nuclei) did not work for human. Unlike in mouse, the DMRT1 protein is

795 expressed in meiotic S-phase (C-E). Thus, some spermatogonia may be present in the MeiS 
796 population. (C) $t$-Distributed Stochastic Neighbor Embedding (t-SNE) projection of the single-cell

797 transcription atlas of human testes (Guo et al., 2018). Each dot represents a single cell. Colors

798 represent defined clusters. (D) Quantification of genes-of-interest in four cell clusters. Circles

799 represent the relative abundance of transcripts. Circle size represents the fraction of cells

800 showing expression. Depth of color represents average gene expression. (E) Single-cell

801 expression patterns of genes-of-interest. Depth of red color indicates expression level $($ Red $=$

802 high; Grey = low). 
bioRxiv preprint doi: https://doi.org/10.1101/2020.09.23.308874; this version posted September 23, 2020. The copyright holder for this preprint (which was not certified by peer review) is the author/funder. This article is a US Government work. It is not subject to copyright under 17 USC 105 and is also made available for use under a CCO license.

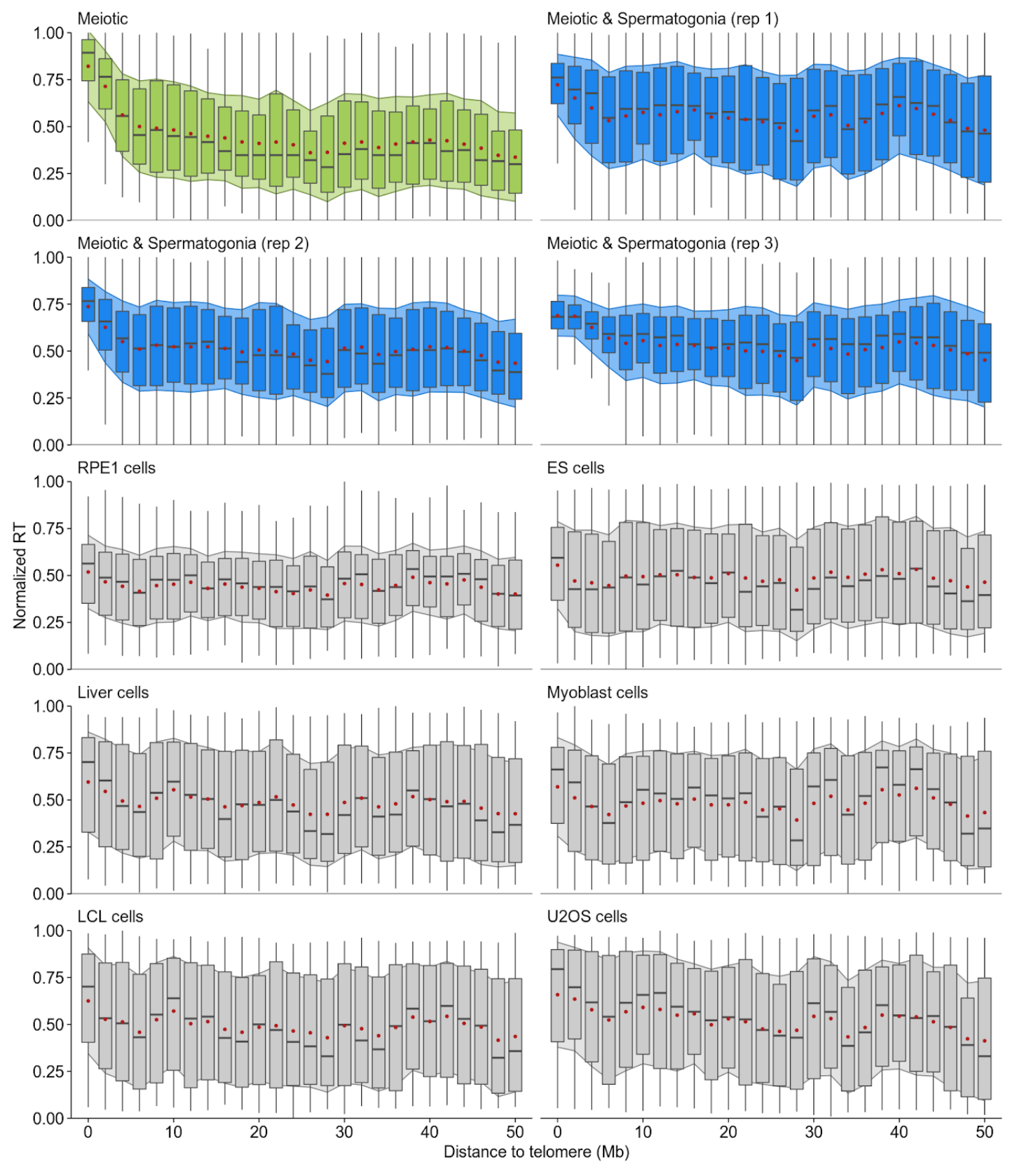

\section{Figure S18. Early sub-telomeric replication is distinct to male meiosis}

804 Consistently early replication in subtelomeric regions is only seen in the germline (green or blue) 805 and not in any of the other cell types studied (grey). The pure meiotic S-phase population 806 (green) exhibits the most pronounced signal. In the three samples which likely contain some 807 spermatogonia (blue), early replication in subtelomeric DNA is seen, but less pronounced. This 
bioRxiv preprint doi: https://doi.org/10.1101/2020.09.23.308874; this version posted September 23, 2020. The copyright holder for this preprint

(which was not certified by peer review) is the author/funder. This article is a US Government work. It is not subject to copyright under 17 USC 105 and is also made available for use under a CCO license.

808 could imply that sub-telomeric DNA does not replicate notably early in spermatogonia. Boxplots

809 depict the range of replication timing values in $2 \mathrm{Mb}$ regions across the genome; the median is

810 shown as a grey bar and the box designates the interquartile range. The mean (red dot) \pm 1

811 standard deviation (filled shadow) is also shown for each interval. Details for each sample are in

812 Table S1, S3. 


\section{Methods}

\section{Tissue sources}

815 C57BL/6J (stock 000664) and CAST/Eij (000928) mice were obtained from The Jackson

816 Laboratory (Maine). Human testicular samples were obtained from a commercial source (Folio,

817 Ohio) that provides testicular biopsies from normal tumor adjacent tissue or from deidentified

818 donor testes from deceased individuals (obtained with the assistance of the Washington

819 Regional Transplant Community).

820 Mapping origins of replication using short nascent-strand capture followed by 821 single-stranded DNA sequencing (Ori-SSDS)

822 For the short nascent strands (SNS) preparation we adapted the protocol from (Picard et al., 823 2014) to work with mouse tissue. Mice were euthanized and testes were retrieved. The tunica 824 albuginea was removed and both testes were suspended in $10 \mathrm{ml}$ of DNAzol (Thermo-Fisher 825 catalog number 10503027) and transferred to a Dounce homogenizer. Tissue and cells were 826 disrupted with 5-7 strokes of a loose-fitting pestle. The DNA was precipitated with $5 \mathrm{ml}$ of EtOH 827 and then spooled with a pipette tip and rinsed gently twice with $\mathrm{EtOH} 75 \%$ by sequential transfer 828 into $15 \mathrm{ml}$ tubes. DNA was air-dried for $5 \mathrm{~min}$ and then resuspended in $500 \mu \mathrm{l}$ of $1 \mathrm{X}$ TEN buffer 829 (Tris $10 \mathrm{mM} \mathrm{pH=8;} \mathrm{EDTA} 1 \mathrm{mM}$; $\mathrm{NaCl} 100 \mathrm{mM}$ ) + $80 \mathrm{U}$ of RNAseOUT (Thermo-Fisher catalog 830 number 10777019$)(40 \mathrm{U} / \mu \mathrm{l})$ at $4^{\circ} \mathrm{C}$ for at least $24 \mathrm{~h}$.

831 DNA was denatured at $95^{\circ} \mathrm{C}$ for $5 \mathrm{~min}$, chilled on ice for $5 \mathrm{~min}$ and size-fractionated on 5 - 30\% $832(\mathrm{w} / \mathrm{v})$ linear sucrose gradients in TEN buffer in a Beckman Ultracentrifuge SW45 rotor for $18 \mathrm{~h}$ at $83326,000 \mathrm{~g}$ at $20^{\circ} \mathrm{C}$. $500 \mathrm{ul}$ fractions were collected from the top of the gradient, precipitated by 834 adding $1 \mathrm{ml}$ of $\mathrm{EtOH} 100 \%$ and $50 \mu \mathrm{l}$ of $\mathrm{NaOAc} 3 \mathrm{M} \mathrm{pH} 5.2$ and resuspended in $25 \mu \mathrm{l}$ Tris Buffer $835 \mathrm{pH}=8$. Fractions were analyzed in a denaturing (50 mM NaOH, $1 \mathrm{mM}$ EDTA) $1.2 \%$ agarose gel 836 and the ones containing ssDNA ranging in size from 800 to $2000 \mathrm{nt}$ were pooled. The sample 837 was then heat-denatured for $5 \mathrm{~min}$ at $95^{\circ} \mathrm{C}$ and chilled on ice for 5 min and DNA was 838 phosphorylated by Polynucleotide Kinase in 150 ul reactions in PNK Buffer (100 U of PNK ; 1X 839 PNK buffer ; $1 \mathrm{mM} \mathrm{ATP;} \mathrm{40U} \mathrm{RNAseOUT).} \mathrm{The} \mathrm{reactions} \mathrm{were} \mathrm{incubated} \mathrm{for} 30 \mathrm{~min}$ at $37^{\circ} \mathrm{C}$ 840 and heat-inactivated at $75^{\circ} \mathrm{C}$ for $15 \mathrm{~min}$. DNA was extracted with Phenol/Chloroform, EtOH 841 precipitated and resuspended in $50 \mu \mathrm{l}$ Tris Buffer $\mathrm{pH}=8$. Samples were heat-denatured again for $8425 \mathrm{~min}$ and digested with lambda-exonuclease in $100 \mu \mathrm{l}$ of custom lambda buffer $(67 \mathrm{mM}$ 
843 glycine- $\mathrm{KOH} \mathrm{pH}=8.8,2.5 \mathrm{mM} \mathrm{MgCl}_{2}, 50 \mu \mathrm{g} / \mathrm{ml} \mathrm{BSA}$ ) with $2.5 \mathrm{U}$ to $10 \mathrm{U}$ lambda-exonuclease $/ \mu \mathrm{g}$ 844 DNA (Thermo Scientific). 80U RNAseOUT, 5U of PNK and $1 \mathrm{mM}$ ATP were added to the 845 sample and incubated overnight at $37^{\circ} \mathrm{C}$. Reactions were inactivated for $10 \mathrm{~min}$ at $75^{\circ} \mathrm{C}$ and 846 DNA was purified with MinElute columns (QIAGEN), as indicated by the instruction manual. 847 RNA primers were hydrolyzed by incubating the eluate for 30 min at $37^{\circ} \mathrm{C}$ in $0.25 \mathrm{~N} \mathrm{NaOH}$, 848 followed by neutralization with acetic acid and purified again with MinElute columns. The sample 849 was then sonicated in a Bioruptor UCD200 for 5 min 30 sec ON 30 sec OFF, "high" setting, and 850 the library was prepared for SSDS as described (Brick et al., 2018b).

851 For one sample (wt_Rep2), separate libraries were made from three fractions from the sucrose 852 gradient (Fraction $10: 800-1,000$ nt, Fraction $11:$ 1,000-1,400 nt and Fraction 12 : 1,400-2,000 853 nt). Sequencing data were subsequently pooled.

854 To validate that the Ori-SSDS enrichment stems from RNA primed leading strands, we 855 performed experiments where we hydrolyzed the RNA prior to lambda exonuclease treatment. 856 This also necessitated changes to the above Ori-SSDS protocol as follows. Selected fractions 857 from the sucrose gradient were split in two tubes. In one tube, leading strand RNA primers were 858 hydrolyzed by incubating for $30 \mathrm{~min}$ at $37^{\circ} \mathrm{C}$ in $0.25 \mathrm{~N} \mathrm{NaOH}$, followed by neutralization with 859 acetic acid. The other tube was incubated for $30 \mathrm{~min}$ at $37^{\circ} \mathrm{C}$ but in Tris Buffer $\mathrm{pH}=8$. After 860 neutralization, both tubes were heat denatured and spun through a Chromaspin TE-1000 size 861 exclusion column (Clontech) to remove any small degradation products that could interfere with 862 later steps. The eluate was then subjected to PNK treatment and subsequent steps outlined 863 above. Two replicates were performed in parallel, with and without RNA hydrolysis (wt_Rep4, 864 wt_Rep5).

865 Ori-SSDS in sperm was used as a negative control as there is no DNA replication. Sperm was 866 retrieved from cauda and caput epididymides from 3 adult mice. The mix of somatic cells and 867 sperm was resuspended in $0.1 \%$ SDS, $0.5 \%$ Triton $X$ to selectively lyse somatic cells. Sperm 868 was recovered by centrifugation and lysed in $6 \mathrm{M}$ guanidinium, $30 \mathrm{mM}$ sodium citrate $(\mathrm{pH} 7.0)$, $8690.5 \%$ Sarkosyl, $0.20 \mathrm{mg} / \mathrm{ml}$ proteinase $\mathrm{K}$ and $0.3 \mathrm{M}$ Beta-mercaptoethanol, and incubated at $87055^{\circ} \mathrm{C}$ for $1 \mathrm{~h}$ (Hossain et al., 1997). The DNA was precipitated with 2 volumes of isopropyl 871 alcohol and then spooled with a pipette tip and rinsed gently twice with $\mathrm{EtOH} 75 \%$ by sequential 872 transfer into $15 \mathrm{ml}$ tubes. DNA was air-dried for $5 \mathrm{~min}$ and then resuspended in $500 \mu \mathrm{l}$ of $1 \mathrm{X}$ 
873 TEN buffer (Tris 10mM pH=8; EDTA $1 \mathrm{mM}$; NaCl 100mM) + $80 \mathrm{U}$ of RNAseOUT (Thermo-Fisher

874 catalog number 10777019$)(40 \mathrm{U} / \mu \mathrm{l})$ at $4^{\circ} \mathrm{C}$ for at least $24 \mathrm{~h}$.

\section{Identification of origins of replication}

876 Sequencing reads from Ori-SSDS experiments were aligned to the reference genome $($ mouse $=$ $877 \mathrm{mm10}$; human = hg38) using bwa 0.7.12 and the single-stranded DNA pipeline (Brick et al., 878 2018b; Khil et al., 2012). Only fragments derived unambiguously from ssDNA (ssDNA_type1) 879 were used in subsequent analyses. ssDNA fragments where either read1 or read2 had mapping 880 quality $<30$ were discarded. Fragments from the mitochondrial chromosome and fragments in 881 blacklisted regions of the genome were also discarded. Duplicate fragments were subsequently 882 discarded.

883 We used the MACS algorithm for peak calling. To assure that data derived from Ori-SSDS fit the 884 MACS peak model, we performed two operations: 1. The strand orientation of fragments was 885 reversed prior to peak calling. 2. The data was passed to MACS as SsDNA fragment BED files 886 (from SSDS pipeline). Peak calling was performed for each Ori-SSDS sample using MACS 8872.1 .2 and the following parameters : -g (hs or mm) -q 0.001 -extsize 2000 --nomodel 888 --nolambda.

889 The final origin set for mouse was defined by merging origin calls from wt_Rep1,2,3. Origin 890 intervals overlapping by $\geq 500$ bp were merged. Origin efficiency was calculated in each sample 891 using a method analogous to that for DMC1-SSDS hotspot mapping (Brick et al., 2018b). 892 Briefly, each origin is re-centred to the midpoint of the Watson and Crick fragment distributions. 893 Signal is estimated as the Crick-strand signal to the left of the origin center + the Watson-strand 894 signal to the right of the origin center. The background is estimated by counting the remaining 895 fragments at the left and right edges of MACS-defined origins (15\%); Watson-strand fragments 896 to the left of the origin center, Crick-strand fragments to the right. This background is then 897 extrapolated to the entire origin and subtracted from the signal. Following this process, origins 898 found in just one sample and origins that did not display the expected Crick/Watson asymmetry 899 (Figure 1A) in any sample were discarded. 


\section{Whole genome sequencing for replication timing}

901 Nuclei from mouse or human testes were prepared as described in (Lam et al., 2019). To purify

902 the populations of interest, a combination of intranuclear markers were used (Table S1, Figure

903 2).

904 Mouse ESC were obtained from Jackson labs: 129X1/SvJ-PRX-129X1 \#1 mES cells derived 905 from day 3.5 blastocysts of strain 129X1/SvJ (Stock Number 000691). The cells were cultured 906 as recommended by Jackson labs. Exponentially growing cells were cross-linked by adding 907 fresh 1\% paraformaldehyde solution to the ES media for 10 minutes at room temperature. The 908 cells were washed twice with $1 \times$ phosphate-buffered saline and scraped off the plates, pelleted 909 and nuclei were prepared as described in (Lam et al., 2019). Replicating cells were sorted 910 based DNA content.

911 Pure nuclei populations were resuspended in $300 \mathrm{ul}$ of lysis buffer (SDS 1\%, 10mM EDTA, $91250 \mathrm{mM}$, Tris $\mathrm{pH}=8$ ) and then sonicated in a Bioruptor UCD200 for 20 min $30 \mathrm{sec}$ ON 30 sec OFF, 913 "high" setting. $\mathrm{NaCl}$ was added to a final concentration of $0.2 \mathrm{M}$ and the sample incubated 914 overnight at $65^{\circ} \mathrm{C}$ to reverse DNA-protein crosslinks. $5 \mathrm{U}$ of Proteinase K (NEB Cat. No. 915 P8107S) was added and the sample further incubated for $1 \mathrm{~h}$ at $45^{\circ} \mathrm{C}$. DNA was purified with 916 MinElute columns (QIAGEN), as indicated by the instruction manual. Whole genome 917 sequencing libraries were prepared from $100 \mathrm{ng}$ of purified DNA using the KAPA Hyper Prep Kit 918 (Cat. No. KK8502) following the manufacturer's instructions.

\section{Inferring replication timing from whole genome sequencing (WGS) data}

920 The mem algorithm of bwa 0.7.12 (Li, 2013; Li and Durbin, 2009) was used to align each WGS 921 dataset to the reference genome (mm10 for mouse; hg38 for human). WGS from a 922 non-replicating population was used to calibrate the expected GC-biases from different 923 sequencing instruments (Illumina HiSeq 2000, Illumina HiSeq 2500, Illumina HiSeq X, Illumina 924 NextSeq 500). Briefly, these calibration files were generated by examining a subset of perfectly 925 mappable genomic loci. Each locus is a 101 bp region with the following properties:

926 1. Full mappability of all bases within the $101 \mathrm{bp}$ window. To determine mappability, we 927 generated a fastq file of pseudoreads of the requisite length from the reference genome 928 fasta file. One pseudo-read was generated per bp; all bases were assigned a q-score of 
60. Pseudo-reads were mapped to the reference genome using bwa mem 0.7.12 and the coverage at each base in the genome was assessed.

2. The window does not overlap with sequencing gaps (UCSC Gaps track), segmental duplications (UCSC SegDups track), high copy repeats (UCSC repeatmasker track) or otherwise blacklisted genomic regions (Amemiya et al., 2019; Kent et al., 2002).

936 for published data are detailed in Table S2 below.

937 The GC content (rounded to 1\%) and WGS sequencing coverage of each $101 \mathrm{bp}$ window was

938 assessed. The median coverage for all windows of a given GC content was used as a 939 correction factor. These correction factors vary substantially across the different sequencing 940 platforms used. $2 \%$ of all qualifying $101 \mathrm{bp}$ autosomal windows were used to calculate the GC 941 correction coefficients.

942 For each RT-Seq experiment, the WGS coverage in 101-bp windows in the genome was 943 calculated and corrected for GC biases using the correction coefficient for the appropriate 944 sequencing platform. Replication timing was calculated as the $\log _{2}$ ratio of corrected sequencing 945 coverage at a locus to the genome-wide median (method was adapted from (Koren et al., 946 2014)). RT-Seq coverage was smoothed in $500 \mathrm{~Kb}$ windows with a $50 \mathrm{~Kb}$ step.

\section{Inferring replication timing from published data}

948 Whole genome sequencing data were obtained from the sources listed in Table S2. Sequencing 949 data were aligned and replication timing profiles were inferred as described above.

\section{Modelling DNA replication}

951 DNA replication was modelled in-silico using custom R code (Brick, 2020). Replication was 952 simulated in a haploid genome. Non-autosomal chromosomes were excluded. The initial wave 953 of origin firing was assumed to be synchronous. $k$ replication origins were selected at random 954 and without replacement, using the origin efficiency as a selection weight. At each origin, a 955 left-ward and a right-ward replication fork was established, and replication was then simulated 956 bi-directionally outward from each origin in $10 \mathrm{~Kb}$ steps. After each step, we assessed which 957 replication forks have collided with another fork and which forks reached the end of the 
958 chromosome. These forks were terminated. For every two terminated forks, we allowed 959 replication to begin from a new, randomly chosen origin (as above, weighted by origin 960 efficiency). Origins that have previously fired and origins in regions that have already been 961 replicated were not considered for selection. This iterative process continued until the genome 962 was fully replicated or for a defined number of cycles. To simulate replication in a population, 963 this simulation was performed in $n$ haploid genomes and the results were combined.

\section{Hyperparameter search for replication models}

965 To assess the best-fitting parameters of DNA replication in-silico, we performed a 966 grid-hyperparameter search. A hyperparameter search was performed for each pair-wise 967 combination of RT and replication origins. We optimized for a scoring function defined as the 968 Pearson correlation coefficient between the experimentally-determined replication timing and 969 the in-silico simulated replication timing. A population of 250 replicating haploid genomes was 970 used for the hyperparameter search. The grid-search generated an RT-Sim model for each 971 combination of the following parameters:

972 a. Origin density; $R_{d}=0.02$ to 0.4 in 0.01 steps; 0.45 to 0.6 in 0.05 steps

973 b. Modelling runtime; $\mathrm{N}_{\text {epochs }}=10$ to 7500 in steps of 10

974 C. Replicating cells in population; $P(\%)=10,30,50,70,90$

975 d. Use origin efficiency; E = TRUE / FALSE (if FALSE, all origins were equally weighted for 976 "random" selection)

977 The fine-grained search yielded multiple models with similar scores, therefore in assessing 978 results, the top $g \%$ of models were examined.

\section{Generating simulated replication timing}

980 The hyperparameters of the top-scoring RT-Sim model were used to generate an RT-Sim model 981 from 500 haploid genomes and run it to completion. Simulated replication timing was then 982 defined as the mean replication time across all simulations for each $10 \mathrm{~Kb}$ window in the 983 genome. 


\section{Sources and processing of annotation data for the mouse genome}

$985 \mathrm{CpG}$ islands in the mouse genome were obtained from the Irizarry lab at Harvard University, 986 USA (http://www.haowulab.org/software/makeCGI/model-based-cpg-islands-mm9.txt) (Wu et 987 al., 2010). UCSC liftover was used to convert $\mathrm{mm} 9$ to $\mathrm{mm} 10$ genome coordinates.

988 Meiotic DSB hotspots in C57BL6J mice were obtained from columns 1,2,3,6 of Supplementary 989 File 41586_2018_492_MOESM3_ESM.zip (Brick et al., 2018a). H3K4m3 ChIP-Seq signals at 990 DSB hotspots in C57BL6J mice (Baker et al., 2014) was obtained from columns 1,2,3,50 of 991 Supplementary File 41586_2018_492_MOESM3_ESM.zip (Brick et al., 2018a). Genome-wide 992 Spo11-associated oligo mapping data in C57BL6 mice (Lange et al., 2016) were obtained in 993 processed form from columns 1,2,3,55 of Supplementary File 994 41586_2018_492_MOESM3_ESM.zip (Brick et al., 2018a). DSB hotspots in Prdm9 ${ }^{-/-}$mice 995 (Brick et al., 2012) were obtained from GSM2664291 (Brick et al., 2018a). DSB hotspots from 996 mice homozygous for a humanized PRDM9 zinc-finger array $\left(B 6^{\mathrm{h} / \mathrm{h}}\right)$ were obtained from the 997 GEO (accession: GSM2049306; file: GSM2049306_dmc1hotspots_B6.PRDM9hh.txt.gz) 998 (Davies et al., 2016). B6 ${ }^{\text {h/h }}$ hotspots overlapping hotspots from Prdm9 ${ }^{-/-}$were discarded. $999 \mathrm{H} 3 \mathrm{~K} 4 \mathrm{~m} 3$ ChIP-Seq peaks in B6 ${ }^{\mathrm{h} / \mathrm{h}}$ mice were obtained from the GEO (accession: GSM1904284; 1000 file GSM1904284_H3K4me3.ForceCalledValues.B6.PRDM9hh.txt) (Davies et al., 2016). 1001 Crossovers and non-crossovers defined by the humanized variant of mouse PRDM9 were 1002 obtained from supplementary file 3 (41467_2019_11675_MOESM6_ESM.csv) and 4 1003 (41467_2019_11675_MOESM7_ESM.csv), respectively, from (Li et al., 2019a). Affinity-Seq 1004 data describing the in-vitro binding preferences of the C57BL6J PRDM9 variant were obtained 1005 from the Sequence Read Archive (accession: SRR1976005) (Walker et al., 2015). Control data 1006 used for Affinity-Seq peak calling was obtained from the Sequence Read Archive (accession: 1007 SRR1976003 \& SRR1976004) (Walker et al. 2015). Affinity-Seq and control reads were aligned 1008 to the reference mm10 genome using bwa mem 0.7.12. Affinity-Seq peak calling was performed 1009 using MACS 2.1.0.20150731 with the following arguments: -g mm -bw 1000 --keep-dup all 1010 --slocal 5000 -q 0.05. Affinity-Seq peak strength was determined as Affinity-Seq coverage less 1011 Control DNA coverage at Affinity-Seq peaks. Hi-C data from mouse zygotene cells was 1012 downloaded from the GEO (GEO accession: GSE122622; file: 1013 GSE122622_zygotene_overall.hic) (Patel et al., 2019). The Juicer tools 1.5 .6 eigenvector 1014 program (Durand et al., 2016) was used to extract the first eigenvector from the .hic file, using 1015 the following arguments: KR, BP, binsize $=100000$, -p. H3K9m2 (accession: SRR1975998) and 
1016 H3K9m3 (accession: SRR1585300) ChIP-Seq data (Walker et al., 2015) from spermatocytes 1017 were obtained from the SRA. Reads were aligned to the reference mm10 genome using bwa 1018 mem 0.7.12.

1019 ATAC-Seq data were obtained from the published datasets described in Supplementary Figure

1020 9. Each dataset was aligned to the mouse mm10 reference genome using bwa mem 0.7.12.

1021 Peak calling was performed for each dataset using MACS 2.1.2 without a control and using 1022 default arguments. ATAC-Seq peaks coinciding with CpG-islands were defined for use on 1023 Supplementary Figure 9.

\section{Mapping Okazaki-fragment sequencing data}

1025 Data from Okazaki-fragment sequencing in mouse ES-cells were obtained from SRA accession 1026 SRR7535256 (Petryk et al., 2018). Sequencing reads were aligned to the $\mathrm{mm} 10$ reference 1027 genome using the mem algorithm of bwa 0.7.12 (Li, 2013; Li and Durbin, 2009).

\section{Obtaining published SNS-Seq replication origins data}

1029 Processed peak calls for MEFs and ESCs were obtained from GEO accession GSE99741

1030 (Almeida et al., 2018); MEFs: GSM2651107_MEFs_WT_repl_I_peaks.bigBed; ESCs: 1031 GSM2651111_mES_WT_repl_I_peaks.bigBed. Processed peak calls for ESCs from (Cayrou et 1032 al., 2015) were obtained from GEO accession GSE68347; 1033 GSE68347_Initiation_Sites.bedGraph.gz. The UCSC liftover tool was used to convert mm9 to $1034 \mathrm{~mm} 10$ coordinates.

\section{Mapping DNA DSB hotspots from DMC1-SSDS experiments}

1036 A testicular biopsy was obtained from a commercial source (Folio, Ohio). Genotyping for 1037 PRDM9 alleles using Sanger sequencing (Pratto et al., 2014) revealed that this individual did 1038 not carry a copy of the most common PRDM9 variant in humans $\left(\right.$ PRDM9 $\left._{A}\right)$. Instead, this 1039 individual was heterozygous for the PRDM9 ${ }_{C}$ and PRDM9 ${ }_{\mathrm{L} 4}$ alleles (Berg et al., 2010). A 1040 DMC1-SSDS library was prepared using the protocol in (Brick et al., 2018b; Pratto et al., 2014).

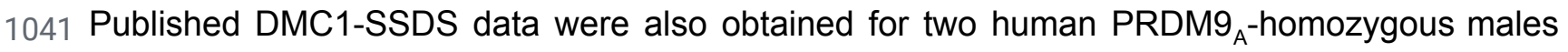
1042 (GEO accessions: GSM1447325 \& GSM1447328) (Pratto et al., 2014). Sequencing reads were 1043 aligned to the human hg38 reference genome using bwa 0.7.12 and the single-stranded DNA 
1044 pipeline (Brick et al., 2018b; Khil et al., 2012). Hotspots were called using the DMC1-SSDS DSB 1045 hotspot calling pipeline (Brick et al., 2018b).

\section{Crossover and non-crossover data for humans}

1047 Crossover data for human males and females were obtained from the supplementary data files 1048 S1 and S2, respectively, from (Halldorsson et al., 2019). Non-crossover data were obtained 1049 from supplementary file S2 from (Halldorsson et al., 2016). NCOs from the ChIP-based 1050 approach and the sequencing-based approach were merged.

\section{Data analysis tools and versions}

1052 BEDtools v.2.29.2 (Quinlan and Hall, 2010)

1053 BWA 0.7.12 (Li, 2013; Li and Durbin, 2009).

1054 DeepTools v.3.0.1 (Ramírez et al., 2014)

1055 Juicer v.1.5.6 (Durand et al., 2016)

1056 MACS v.2.1.0.20150731 (Zhang et al., 2008)

1057 MACS v.2.1.2 (Zhang et al., 2008)

1058 Nextflow v.20.01.0 (Di Tommaso et al., 2017)

1059 Picard v.2.9.2 (Broad Institute of MIT and Harvard, 2018)

1060 R v.3.6.0 (R Core Team, 2014)

1061 SAMtools v.1.9 (Li et al., 2009)

1062 SRA toolkit v.2.9.2 (Leinonen et al., 2011)

1063 UCSC toolkit v.385 (Kent et al., 2010) 


\section{References}

1065 Almeida, R., Fernández-Justel, J.M., Santa-María, C., Cadoret, J.-C., Cano-Aroca, L., 1066 Lombraña, R., Herranz, G., Agresti, A., and Gómez, M. (2018). Chromatin conformation 1067 regulates the coordination between DNA replication and transcription. Nat. Commun. 9, 1590.

1068 Amemiya, H.M., Kundaje, A., and Boyle, A.P. (2019). The ENCODE Blacklist: Identification of 1069 Problematic Regions of the Genome. Sci. Rep. 9, 9354.

1070 Baker, C.L., Walker, M., Kajita, S., Petkov, P.M., and Paigen, K. (2014). PRDM9 binding 1071 organizes hotspot nucleosomes and limits Holliday junction migration. Genome Res. 24, 1072 724-732.

1073 Baudat, F., Buard, J., Grey, C., Fledel-Alon, A., Ober, C., Przeworski, M., Coop, G., and de 1074 Massy, B. (2010). PRDM9 is a major determinant of meiotic recombination hotspots in humans 1075 and mice. Science 327, 836-840.

1076 Bellve, A.R., Cavicchia, J.C., and Millette, C.F. (1977). Spermatogenic cells of the prepuberal 1077 mouse: isolation and morphological characterization. J. Cell Biol.

1078 Bennett, M.D., Smith, J.B., and Riley, R. (1972). The effects of polyploidy on meiotic duration 1079 and pollen development in cereal anthers. Proceedings of the Royal Society of London. Series

1080 B. Biological Sciences 181, 81-107.

1081 Berg, I.L., Neumann, R., Lam, K.-W.G., Sarbajna, S., Odenthal-Hesse, L., May, C.A., and 1082 Jeffreys, A.J. (2010). PRDM9 variation strongly influences recombination hot-spot activity and 1083 meiotic instability in humans. Nat. Genet. 42, 859-863.

1084 Bergerat, A., de Massy, B., Gadelle, D., Varoutas, P.C., Nicolas, A., and Forterre, P. (1997). An 1085 atypical topoisomerase II from Archaea with implications for meiotic recombination. Nature 386, 1086 414-417.

1087 Bielinsky, A.K., and Gerbi, S.A. (1998). Discrete start sites for DNA synthesis in the yeast ARS1 1088 origin. Science 279, 95-98.

1089 Bishop, D.K., Park, D., Xu, L., and Kleckner, N. (1992). DMC1: a meiosis-specific yeast 1090 homolog of $\mathrm{E}$. coli recA required for recombination, synaptonemal complex formation, and cell 1091 cycle progression. Cell 69, 439-456.

1092 Blitzblau, H.G., Chan, C.S., Hochwagen, A., and Bell, S.P. (2012). Separation of DNA 1093 replication from the assembly of break-competent meiotic chromosomes. PLoS Genet. 8, 1094 e1002643.

1095 Borde, V., Goldman, A.S., and Lichten, M. (2000). Direct coupling between meiotic DNA 1096 replication and recombination initiation. Science 290, 806-809.

1097 Boulton, A., Myers, R.S., and Redfield, R.J. (1997). The hotspot conversion paradox and the 
1098 evolution of meiotic recombination. Proc. Natl. Acad. Sci. U. S. A. 94, 8058-8063.

1099 Brick, K. (2020). Simulating replication timing in the mouse and human genomes (Github).

1100 Brick, K., Smagulova, F., Khil, P., Camerini-Otero, R.D., and Petukhova, G.V. (2012). Genetic

1101 recombination is directed away from functional genomic elements in mice. Nature 485,

1102 642-645.

1103 Brick, K., Thibault-Sennett, S., Smagulova, F., Lam, K.-W.G., Pu, Y., Pratto, F., Camerini-Otero, 1104 R.D., and Petukhova, G.V. (2018a). Extensive sex differences at the initiation of genetic

1105 recombination. Nature 561, 338-342.

1106 Brick, K., Pratto, F., Sun, C.-Y., Camerini-Otero, R.D., and Petukhova, G. (2018b). Analysis of

1107 Meiotic Double-Strand Break Initiation in Mammals. In Methods in Enzymology, (Academic

1108 Press), pp. 391-418.

1109 Broach, J.R., Li, Y.Y., Feldman, J., Jayaram, M., Abraham, J., Nasmyth, K.A., and Hicks, J.B.

1110 (1983). Localization and sequence analysis of yeast origins of DNA replication. Cold Spring

1111 Harb. Symp. Quant. Biol. 47 Pt 2, 1165-1173.

1112 Broad Institute of MIT and Harvard (2018). Picard tools (Broad Institute of MIT and Harvard).

1113 Buard, J., Rivals, E., Dunoyer de Segonzac, D., Garres, C., Caminade, P., de Massy, B., and

1114 Boursot, P. (2014). Diversity of Prdm9 zinc finger array in wild mice unravels new facets of the 1115 evolutionary turnover of this coding minisatellite. PLoS One 9, e85021.

1116 Callan, H.G. (1973). DNA Replication in the Chromosomes of Eukaryotes. In Molecular 1117 Cytogenetics, B.A. Hamkalo, and J. Papaconstantinou, eds. (Boston, MA: Springer New York), 1118 pp. 31-47.

1119 Castro, J.P.L., Yancoskie, M.N., Marchini, M., Belohlavy, S., Hiramatsu, L., Kučka, M., Beluch, 1120 W.H., Naumann, R., Skuplik, I., Cobb, J., et al. (2019). An integrative genomic analysis of the 1121 Longshanks selection experiment for longer limbs in mice. eLife 8.

1122 Cayrou, C., Grégoire, D., Coulombe, P., Danis, E., and Méchali, M. (2012). Genome-scale 1123 identification of active DNA replication origins. Methods 57, 158-164.

1124 Cayrou, C., Ballester, B., Peiffer, I., Fenouil, R., Coulombe, P., Andrau, J.-C., van Helden, J., 1125 and Méchali, M. (2015). The chromatin environment shapes DNA replication origin organization 1126 and defines origin classes. Genome Res. 25, 1873-1885.

1127 Cha, R.S., and Kleckner, N. (2002). ATR homolog Mec1 promotes fork progression, thus 1128 averting breaks in replication slow zones. Science 297, 602-606.

1129 Cha, R.S., Weiner, B.M., Keeney, S., Dekker, J., and Kleckner, N. (2000). Progression of meiotic 1130 DNA replication is modulated by interchromosomal interaction proteins, negatively by Spo $11 \mathrm{p}$

1131 and positively by Rec8p. Genes Dev. 14, 493-503.

1132 Chagin, V.O., Casas-Delucchi, C.S., Reinhart, M., Schermelleh, L., Markaki, Y., Maiser, A., 1133 Bolius, J.J., Bensimon, A., Fillies, M., Domaing, P., et al. (2016). 4D Visualization of replication 
1134 foci in mammalian cells corresponding to individual replicons. Nat. Commun. 7, 11231.

1135 Chandley, A.C. (1986). A model for effective pairing and recombination at meiosis based on 1136 early replicating sites (R-bands) along chromosomes. Hum. Genet. 72, 50-57.

1137 Davies, B., Hatton, E., Altemose, N., Hussin, J.G., Pratto, F., Zhang, G., Hinch, A.G., Moralli, D., 1138 Biggs, D., Diaz, R., et al. (2016). Re-engineering the zinc fingers of PRDM9 reverses hybrid

1139 sterility in mice. Nature 530, 171-176.

1140 Diagouraga, B., Clément, J.A.J., Duret, L., Kadlec, J., de Massy, B., and Baudat, F. (2018).

1141 PRDM9 Methyltransferase Activity Is Essential for Meiotic DNA Double-Strand Break Formation

1142 at Its Binding Sites. Mol. Cell.

1143 Dileep, V., and Gilbert, D.M. (2018). Single-cell replication profiling to measure stochastic 1144 variation in mammalian replication timing. Nat. Commun. 9, 427.

1145 Di Tommaso, P., Chatzou, M., Floden, E.W., Barja, P.P., Palumbo, E., and Notredame, C.

1146 (2017). Nextflow enables reproducible computational workflows. Nat. Biotechnol. 35, 316-319.

1147 Durand, N.C., Shamim, M.S., Machol, I., Rao, S.S.P., Huntley, M.H., Lander, E.S., and Aiden,

1148 E.L. (2016). Juicer Provides a One-Click System for Analyzing Loop-Resolution Hi-C

1149 Experiments. Cell Syst 3, 95-98.

1150 Duret, L., and Galtier, N. (2009). Biased gene conversion and the evolution of mammalian 1151 genomic landscapes. Annu. Rev. Genomics Hum. Genet. 10, 285-311.

1152 Foulk, M.S., Urban, J.M., Casella, C., and Gerbi, S.A. (2015). Characterizing and controlling 1153 intrinsic biases of lambda exonuclease in nascent strand sequencing reveals phasing between 1154 nucleosomes and G-quadruplex motifs around a subset of human replication origins. Genome 1155 Res. 25, 725-735.

1156 Fu, H., Besnard, E., Desprat, R., Ryan, M., Kahli, M., Lemaitre, J.-M., and Aladjem, M.I. (2014). 1157 Mapping replication origin sequences in eukaryotic chromosomes. Curr. Protoc. Cell Biol. 65, 1158 22-20.

1159 Ghosal, S.K., and Mukherjee, B.B. (1971). THE CHRONOLOGY OF DNA SYNTHESIS, 1160 MEIOSIS AND SPERMIOGENESIS IN THE MALE MOUSE AND GOLDEN HAMSTER. Can. J. 1161 Genet. Cytol. 13, 672-682.

1162 Gindin, Y., Valenzuela, M.S., Aladjem, M.I., Meltzer, P.S., and Bilke, S. (2014). A chromatin 1163 structure-based model accurately predicts DNA replication timing in human cells. Mol. Syst. 1164 Biol. 10, 722.

1165 Goodsell, D.S., and Dickerson, R.E. (1994). Bending and curvature calculations in B-DNA. 1166 Nucleic Acids Res. 22, 5497-5503.

1167 Grant, G.D., Kedziora, K.M., Limas, J.C., Cook, J.G., and Purvis, J.E. (2018). Accurate 1168 delineation of cell cycle phase transitions in living cells with PIP-FUCCl. Cell Cycle 17, 1169 2496-2516. 
1170 Guo, J., Grow, E.J., Mlcochova, H., Maher, G.J., Lindskog, C., Nie, X., Guo, Y., Takei, Y., Yun, 1171 J., Cai, L., et al. (2018). The adult human testis transcriptional cell atlas. Cell Res. 28,

1172 1141-1157.

1173 Halldorsson, B.V., Hardarson, M.T., Kehr, B., Styrkarsdottir, U., Gylfason, A., Thorleifsson, G., 1174 Zink, F., Jonasdottir, A., Jonasdottir, A., Sulem, P., et al. (2016). The rate of meiotic gene 1175 conversion varies by sex and age. Nat. Genet. 48, 1377-1384.

1176 Halldorsson, B.V., Palsson, G., Stefansson, O.A., Jonsson, H., Hardarson, M.T., Eggertsson, 1177 H.P., Gunnarsson, B., Oddsson, A., Halldorsson, G.H., Zink, F., et al. (2019). Characterizing 1178 mutagenic effects of recombination through a sequence-level genetic map. Science 363.

1179 Handel, M.A., Eppig, J.J., and Schimenti, J.C. (2014). Applying "Gold Standards" to 1180 In-Vitro-Derived Germ Cells. Cell 157, 1257-1261.

1181 Higgins, J.D., Perry, R.M., Barakate, A., Ramsay, L., Waugh, R., Halpin, C., Armstrong, S.J., 1182 and Franklin, F.C.H. (2012). Spatiotemporal asymmetry of the meiotic program underlies the 1183 predominantly distal distribution of meiotic crossovers in barley. Plant Cell 24, 4096-4109.

1184 Hinch, A.G., Zhang, G., Becker, P.W., Moralli, D., Hinch, R., Davies, B., Bowden, R., and 1185 Donnelly, P. (2019). Factors influencing meiotic recombination revealed by whole-genome 1186 sequencing of single sperm. Science 363, eaau8861.

1187 Hiratani, I., Ryba, T., Itoh, M., Rathjen, J., Kulik, M., Papp, B., Fussner, E., Bazett-Jones, D.P., 1188 Plath, K., Dalton, S., et al. (2010). Genome-wide dynamics of replication timing revealed by in

1189 vitro models of mouse embryogenesis. Genome Res. 20, 155-169.

1190 Holm, P.B. (1977). The premeiotic DNA replication of euchromatin and heterochromatin in Lilium 1191 longiflorum (Thunb.). Carlsberg Res. Commun. 42, 249-281.

1192 Hossain, A.M., Rizk, B., Behzadian, A., and Thorneycroft, I.H. (1997). Modified guanidinium

1193 thiocyanate method for human sperm DNA isolation. Mol. Hum. Reprod. 3, 953-956.

1194 Huppert, J.L., and Balasubramanian, S. (2005). Prevalence of quadruplexes in the human

1195 genome. Nucleic Acids Res. 33, 2908-2916.

1196 Jodkowska, K., Pancaldi, V., Almeida, R., Rigau, M., Graña-Castro, O., Fernández-Justel, J.M., 1197 Rodríguez-Acebes, S., Rubio-Camarillo, M., Pau, E.C.S., Pisano, D., et al. (2019).

1198 Three-dimensional connectivity and chromatin environment mediate the activation efficiency of 1199 mammalian DNA replication origins.

1200 Joshi, N., Brown, M.S., Bishop, D.K., and Börner, G.V. (2015). Gradual implementation of the 1201 meiotic recombination program via checkpoint pathways controlled by global DSB levels. Mol. 1202 Cell 57, 797-811.

1203 Kaback, D.B., Guacci, V., Barber, D., and Mahon, J.W. (1992). Chromosome size-dependent 1204 control of meiotic recombination. Science 256, 228-232.

1205 Keeney, S., Giroux, C.N., and Kleckner, N. (1997). Meiosis-specific DNA double-strand breaks 
1206 are catalyzed by Spo11, a member of a widely conserved protein family. Cell 88, 375-384.

1207 Kelly, T., and Callegari, A.J. (2019). Dynamics of DNA replication in a eukaryotic cell. Proc. Natl.

1208 Acad. Sci. U. S. A. 116, 4973-4982.

1209 Kent, W.J., Sugnet, C.W., Furey, T.S., Roskin, K.M., Pringle, T.H., Zahler, A.M., and Haussler, D.

1210 (2002). The human genome browser at UCSC. Genome Res. 12, 996-1006.

1211 Kent, W.J., Zweig, A.S., Barber, G., Hinrichs, A.S., and Karolchik, D. (2010). BigWig and

1212 BigBed: enabling browsing of large distributed datasets. Bioinformatics 26, 2204-2207.

1213 Khil, P.P., Smagulova, F., Brick, K.M., Camerini-Otero, R.D., and Petukhova, G.V. (2012).

1214 Sensitive mapping of recombination hotspots using sequencing-based detection of ssDNA.

1215 Genome Res. 22, gr - 130583.

1216 Klein, K.N., Zhao, P.A., Lyu, X., Bartlett, D.A., Singh, A., Tasan, I., Watts, L.P., Hiraga, S.-I.,

1217 Natsume, T., Zhou, X., et al. (2019). Replication timing maintains the global epigenetic state in

1218 human cells.

1219 Kofman-Alfaro, S., and Chandley, A.C. (1970). Meiosis in the male mouse. An autoradiographic

1220 investigation. Chromosoma 31, 404-420.

1221 Kojima, M.L., de Rooij, D.G., and Page, D.C. (2019). Amplification of a broad transcriptional

1222 program by a common factor triggers the meiotic cell cycle in mice. Elife 8.

1223 Koren, A., Polak, P., Nemesh, J., Michaelson, J.J., Sebat, J., Sunyaev, S.R., and McCarroll, S.A. 1224 (2012). Differential relationship of DNA replication timing to different forms of human mutation 1225 and variation. Am. J. Hum. Genet. 91, 1033-1040.

1226 Koren, A., Handsaker, R.E., Kamitaki, N., Karlić, R., Ghosh, S., Polak, P., Eggan, K., and 1227 McCarroll, S.A. (2014). Genetic variation in human DNA replication timing. Cell 159,

1228 1015-1026.

1229 Kulakovskiy, I.V., Vorontsov, I.E., Yevshin, I.S., Sharipov, R.N., Fedorova, A.D., Rumynskiy, E.I., 1230 Medvedeva, Y.A., Magana-Mora, A., Bajic, V.B., Papatsenko, D.A., et al. (2018). HOCOMOCO: 1231 towards a complete collection of transcription factor binding models for human and mouse via 1232 large-scale ChIP-Seq analysis. Nucleic Acids Res. 46, D252-D259.

1233 Lam, K.-W.G., Brick, K., Cheng, G., Pratto, F., and Camerini-Otero, R.D. (2019).

1234 Cell-type-specific genomics reveals histone modification dynamics in mammalian meiosis. Nat.

1235 Commun. 10, 3821.

1236 Lange, J., Yamada, S., Tischfield, S.E., Pan, J., Kim, S., Zhu, X., Socci, N.D., Jasin, M., and

1237 Keeney, S. (2016). The Landscape of Mouse Meiotic Double-Strand Break Formation,

1238 Processing, and Repair. Cell 167, 695-708.e16.

1239 Lee, B., and Amon, A. (2001). Meiosis: how to create a specialized cell cycle. Curr. Opin. Cell 1240 Biol. 13, 770-777.

1241 Leinonen, R., Sugawara, H., Shumway, M., and International Nucleotide Sequence Database 
1242 Collaboration (2011). The sequence read archive. Nucleic Acids Res. 39, D19-D21.

$1243 \mathrm{Li}, \mathrm{H}$. (2013). Aligning sequence reads, clone sequences and assembly contigs with BWA-MEM.

$1244 \mathrm{Li}, \mathrm{H}$., and Durbin, R. (2009). Fast and accurate short read alignment with Burrows-Wheeler

1245 transform. Bioinformatics 25, 1754-1760.

1246 Li, H., Handsaker, B., Wysoker, A., Fennell, T., Ruan, J., Homer, N., Marth, G., Abecasis, G., 1247 Durbin, R., and 1000 Genome Project Data Processing Subgroup (2009). The Sequence

1248 Alignment/Map format and SAMtools. Bioinformatics 25, 2078-2079.

1249 Li, N., Lam, W.H., Zhai, Y., Cheng, J., Cheng, E., Zhao, Y., Gao, N., and Tye, B.-K. (2018).

1250 Structure of the origin recognition complex bound to DNA replication origin. Nature 559,

1251 217-222.

1252 Li, R., Bitoun, E., Altemose, N., Davies, R.W., Davies, B., and Myers, S.R. (2019a). A

1253 high-resolution map of non-crossover events reveals impacts of genetic diversity on mammalian

1254 meiotic recombination. Nat. Commun. 10, 1-15.

1255 Li, W., Yang, L., He, Q., Hu, C., Zhu, L., Ma, X., Ma, X., Bao, S., Li, L., Chen, Y., et al. (2019b).

1256 A Homeostatic Arid1a-Dependent Permissive Chromatin State Licenses Hepatocyte

1257 Responsiveness to Liver-Injury-Associated YAP Signaling. Cell Stem Cell 25, 54-68.e5.

1258 Long, H., Zhang, L., Lv, M., Wen, Z., Zhang, W., Chen, X., Zhang, P., Li, T., Chang, L., Jin, C., et 1259 al. (2020). H2A.Z facilitates licensing and activation of early replication origins. Nature 577 ,

$1260576-581$.

1261 Maezawa, S., Yukawa, M., Alavattam, K.G., Barski, A., and Namekawa, S.H. (2018). Dynamic 1262 reorganization of open chromatin underlies diverse transcriptomes during spermatogenesis.

1263 Nucleic Acids Res. 46, 593-608.

1264 Marchal, C., Sima, J., and Gilbert, D.M. (2019). Control of DNA replication timing in the 3D 1265 genome. Nat. Rev. Mol. Cell Biol. 20, 721-737.

1266 Miotto, B., Ji, Z., and Struhl, K. (2016). Selectivity of ORC binding sites and the relation to 1267 replication timing, fragile sites, and deletions in cancers. Proc. Natl. Acad. Sci. U. S. A. 113, 1268 E4810-E4819.

1269 Monesi, V. (1962). Autoradiographic study of DNA synthesis and the cell cycle in spermatogonia 1270 and spermatocytes of mouse testis using tritiated thymidine. J. Cell Biol. 14, 1-18.

1271 Murakami, H., and Keeney, S. (2014). Temporospatial coordination of meiotic DNA replication

1272 and recombination via DDK recruitment to replisomes. Cell 158, 861-873.

1273 Murakami, H., and Nurse, P. (2001). Regulation of premeiotic S phase and

1274 recombination-related double-strand DNA breaks during meiosis in fission yeast. Nat. Genet.

1275 28, 290-293.

1276 Murakami, H., Lam, I., Huang, P.-C., Song, J., van Overbeek, M., and Keeney, S. (2020).

1277 Multilayered mechanisms ensure that short chromosomes recombine in meiosis. Nature 582, 
1278 124-128.

1279 Myers, S., Bottolo, L., Freeman, C., McVean, G., and Donnelly, P. (2005). A fine-scale map of

1280 recombination rates and hotspots across the human genome. Science 310, 321-324.

1281 Myers, S., Bowden, R., Tumian, A., Bontrop, R.E., Freeman, C., MacFie, T.S., McVean, G., and 1282 Donnelly, P. (2010). Drive against hotspot motifs in primates implicates the PRDM9 gene in 1283 meiotic recombination. Science $327,876-879$.

1284 Neale, M.J., Pan, J., and Keeney, S. (2005). Endonucleolytic processing of covalent

1285 protein-linked DNA double-strand breaks. Nature 436, 1053-1057.

1286 Parvanov, E.D., Petkov, P.M., and Paigen, K. (2010). Prdm9 controls activation of mammalian

1287 recombination hotspots. Science $327,835$.

1288 Patel, L., Kang, R., Rosenberg, S.C., Quu, Y., Raviram, R., Chee, S., Hu, R., Ren, B., Cole, F., 1289 and Corbett, K.D. (2019). Dynamic reorganization of the genome shapes the recombination 1290 landscape in meiotic prophase. Nat. Struct. Mol. Biol. 1.

1291 Petryk, N., Kahli, M., d’Aubenton-Carafa, Y., Jaszczyszyn, Y., Shen, Y., Silvain, M., Thermes, C., 1292 Chen, C.-L., and Hyrien, O. (2016). Replication landscape of the human genome. Nat.

1293 Commun. 7, 10208.

1294 Petryk, N., Dalby, M., Wenger, A., Stromme, C.B., Strandsby, A., Andersson, R., and Groth, A. 1295 (2018). MCM2 promotes symmetric inheritance of modified histones during DNA replication.

1296 Science 361, 1389-1392.

1297 Picard, F., Cadoret, J.-C., Audit, B., Arneodo, A., Alberti, A., Battail, C., Duret, L., and Prioleau, 1298 M.-N. (2014). The spatiotemporal program of DNA replication is associated with specific

1299 combinations of chromatin marks in human cells. PLoS Genet. 10, e1004282.

1300 Powers, N.R., Parvanov, E.D., Baker, C.L., Walker, M., Petkov, P.M., and Paigen, K. (2016). The 1301 Meiotic Recombination Activator PRDM9 Trimethylates Both H3K36 and H3K4 at

1302 Recombination Hotspots In Vivo. PLoS Genet. 12, e1006146.

1303 Pratto, F., Brick, K., Khil, P., Smagulova, F., Petukhova, G.V., and Camerini-Otero, R.D. (2014).

1304 Recombination initiation maps of individual human genomes. Science 346, 1256442.

1305 Quinlan, A.R., and Hall, I.M. (2010). BEDTools: a flexible suite of utilities for comparing genomic 1306 features. Bioinformatics 26, 841-842.

1307 Ramírez, F., Dündar, F., Diehl, S., Grüning, B.A., and Manke, T. (2014). deepTools: a flexible 1308 platform for exploring deep-sequencing data. Nucleic Acids Res. 42, W187-W191.

1309 R Core Team (2014). R: A language and environment for statistical computing (R Foundation for 1310 Statistical Computing, Vienna, Austria.).

1311 Sandhu, R., Monge Neria, F., Monge Neria, J., Chen, X., Hollingsworth, N.M., and Börner, G.V. 1312 (2020). DNA Helicase Mph1FANCM Ensures Meiotic Recombination between Parental 
1313 Chromosomes by Dissociating Precocious Displacement Loops. Dev. Cell 53, 458-472.e5.

1314 Sequeira-Mendes, J., Vergara, Z., Peiró, R., Morata, J., Aragüez, I., Costas, C.,

1315 Mendez-Giraldez, R., Casacuberta, J.M., Bastolla, U., and Gutierrez, C. (2019). Differences in

1316 firing efficiency, chromatin, and transcription underlie the developmental plasticity of the

1317 Arabidopsis DNA replication origins. Genome Res. 29, 784-797.

1318 Smagulova, F., Gregoretti, I.V., Brick, K., Khil, P., Camerini-Otero, R.D., and Petukhova, G.V.

1319 (2011). Genome-wide analysis reveals novel molecular features of mouse recombination

1320 hotspots. Nature 472, 375-378.

1321 Smagulova, F., Brick, K., Pu, Y., Camerini-Otero, R.D., and Petukhova, G.V. (2016). The

1322 evolutionary turnover of recombination hot spots contributes to speciation in mice. Genes Dev.

1323 30, 266-280.

1324 Smith, D.I., Zhu, Y., McAvoy, S., and Kuhn, R. (2006). Common fragile sites, extremely large 1325 genes, neural development and cancer. Cancer Lett. 232, 48-57.

1326 Smith, O.K., Kim, R., Fu, H., Martin, M.M., Lin, C.M., Utani, K., Zhang, Y., Marks, A.B., Lalande, 1327 M., Chamberlain, S., et al. (2016). Distinct epigenetic features of differentiation-regulated

1328 replication origins. Epigenetics Chromatin 9, 18.

1329 Stinchcomb, D.T., Thomas, M., Kelly, J., Selker, E., and Davis, R.W. (1980). Eukaryotic DNA

1330 segments capable of autonomous replication in yeast. Proc. Natl. Acad. Sci. U. S. A. 77,

1331 4559-4563.

1332 Takahashi, S., Miura, H., Shibata, T., Nagao, K., Okumura, K., Ogata, M., Obuse, C.,

1333 Takebayashi, S.-I., and Hiratani, I. (2019). Genome-wide stability of the DNA replication

1334 program in single mammalian cells. Nat. Genet. 51, 529-540.

1335 Valton, A.-L., Hassan-Zadeh, V., Lema, I., Boggetto, N., Alberti, P., Saintomé, C., Riou, J.-F.,

1336 and Prioleau, M.-N. (2014). G4 motifs affect origin positioning and efficiency in two vertebrate

1337 replicators. EMBO J. 33, 732-746.

1338 Walker, M., Billings, T., Baker, C.L., Powers, N., Tian, H., Saxl, R.L., Choi, K., Hibbs, M.A., 1339 Carter, G.W., Handel, M.A., et al. (2015). Affinity-seq detects genome-wide PRDM9 binding 1340 sites and reveals the impact of prior chromatin modifications on mammalian recombination 1341 hotspot usage. Epigenetics Chromatin 8, 31.

1342 Wu, P.-Y.J., and Nurse, P. (2014). Replication origin selection regulates the distribution of 1343 meiotic recombination. Mol. Cell 53, 655-662.

$1344 \mathrm{Wu}$, H., Caffo, B., Jaffee, H.A., Irizarry, R.A., and Feinberg, A.P. (2010). Redefining CpG islands 1345 using hidden Markov models. Biostatistics 11, 499-514.

1346 Yevshin, I., Sharipov, R., Valeev, T., Kel, A., and Kolpakov, F. (2017). GTRD: a database of 1347 transcription factor binding sites identified by ChIP-seq experiments. Nucleic Acids Res. 45, 1348 D61-D67.

1349 Yin, Y., Jiang, Y., Lam, K.-W.G., Berletch, J.B., Disteche, C.M., Noble, W.S., Steemers, F.J., 
bioRxiv preprint doi: https://doi.org/10.1101/2020.09.23.308874; this version posted September 23, 2020. The copyright holder for this preprint (which was not certified by peer review) is the author/funder. This article is a US Government work. It is not subject to copyright under 17 USC 105 and is also made available for use under a CCO license.

1350 Camerini-Otero, R.D., Adey, A.C., and Shendure, J. (2019). High-Throughput Single-Cell 1351 Sequencing with Linear Amplification. Mol. Cell.

1352 Zhang, Y., Liu, T., Meyer, C.A., Eeckhoute, J., Johnson, D.S., Bernstein, B.E., Nusbaum, C., 1353 Myers, R.M., Brown, M., Li, W., et al. (2008). Model-based analysis of ChIP-Seq (MACS). 1354 Genome Biol. 9, R137. 